d..

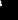

4 $\quad 1$

\title{
MEASURINC AND PREDICTING THE DYNAMIC EFFECTS OF A CONFINED THIN METAL PLATE PILSE HEATED INTO THE LIQUID-VAPOR REGIME
}

\author{
R. C. Baxter
}

December 31, 1977

Prepared for U.S. Energy Research \& Development Administration under contract No. W-7405-Eng-48
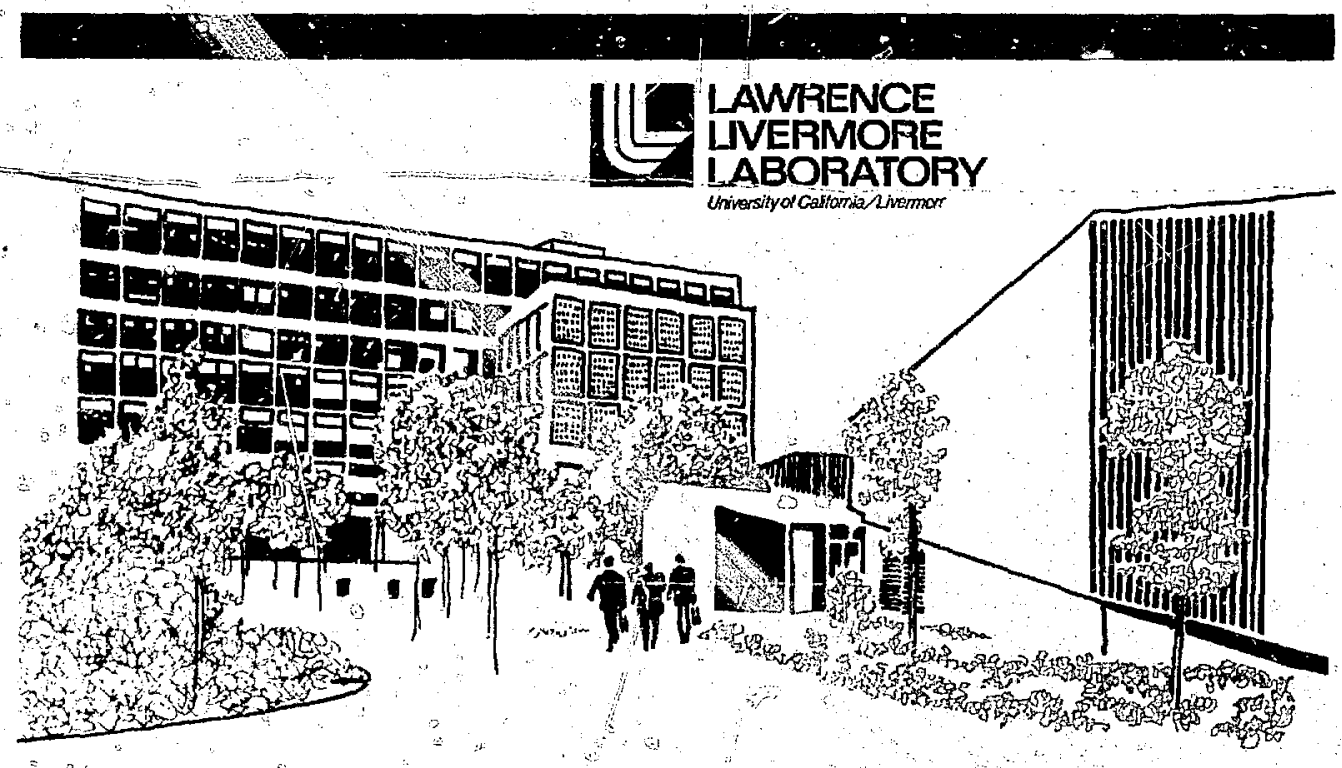


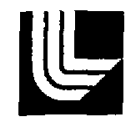

\title{
LAWRENCE LIVEPMORE LABORATORY
}

University of Calfornia Livermore, California 94550

\section{MEASURING AND PREDICTING T HE \\ DYNAMIC EFFECTS OF A CONFIN ED \\ THIN METAL PLATE PULSE HEATED \\ INTO THE LIQUID-VAPOR REGIME}

\author{
R. C. Baxter \\ (D. Engr. Dissertation)
}

MS. date: December 31, 1977

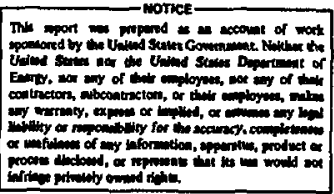




\section{TABLE OF CONTENTS}

ABSTRACT $\ldots \ldots \ldots \ldots \ldots \ldots \ldots \ldots \ldots \ldots \ldots \ldots \ldots \ldots \ldots \ldots \ldots \ldots \ldots \ldots \ldots \ldots$

I. INTRODUCTION $\ldots \ldots \ldots \ldots \ldots \ldots \ldots \ldots \ldots \ldots \ldots \ldots \ldots \ldots \ldots \ldots \ldots \ldots$,

II. EXPERIMENTAL APPARATUS $\ldots \ldots \ldots \ldots \ldots \ldots \ldots \ldots \ldots \ldots \ldots \ldots, 7$

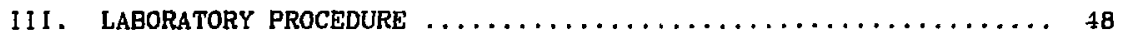

IV. EXPERIMENTAL RESULTS $\ldots \ldots \ldots \ldots \ldots \ldots \ldots \ldots \ldots \ldots \ldots \ldots \ldots \ldots \ldots \ldots \ldots$

v. PREDICTED RESULTS $\ldots \ldots \ldots \ldots \ldots \ldots \ldots \ldots \ldots \ldots \ldots \ldots \ldots \ldots \ldots$ ro

VI. DISCUSSION JF EXPERIMENTAL AND PREDICTED RESULTS $\ldots \ldots \ldots \ldots$. 89

VII. CONCLUSIONS AND RECOMMENDATIONS $\ldots \ldots \ldots \ldots \ldots \ldots \ldots \ldots \ldots \ldots$

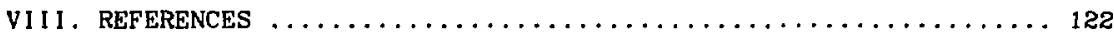

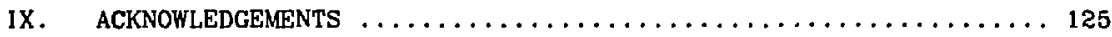

X. APPENDICES

A. Nomenc lature $\ldots \ldots \ldots \ldots \ldots \ldots \ldots \ldots \ldots \ldots \ldots \ldots \ldots \ldots \ldots, 126$

B. Mechanical Analogue of the Pulsed Electron Beam $\ldots \ldots \ldots \ldots 130$

C. Sumnary of Computer Programs Used for Data Interpretation ... 148

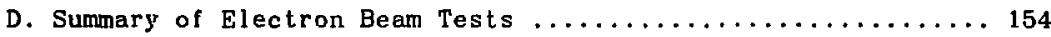




\section{ABSTRACT}

The dynamic response of a coniined thin layer of lead heated rapidly and uniformly to a supercritical state was investigated. Lead targets $0.025 \mathrm{~mm}$ and $0.05 \mathrm{~mm}$ thick were contained between a thin titanium tamping layer and a thick layer of fused quartz with several different gap widths between the lead and the confining surfaces. After being heated by an electron beam for about 50 nanoseconds, lead specimens expanded to a state of approximately half liquid and half vapor. Measurements of the stress in the quartz and the velocity of the tamper produced by the expanding lead were compared with one dimensional hydrodynamic computer program predictions. Measured and predicted peak stresses in the quartz for no gaps were approximately $12 \mathrm{kilobars}$ and agreed within one kilobar. Peak stresses decreased rapidly with gap size to values, at $0.02 \mathrm{~mm}$ gaps, of about one kilobar for the $0.025 \mathrm{~mm}$ lead targets and $f$ ive kilobars for the $0.05 \mathrm{~mm}$ targets. These values were confirmed by measurements. Predictions and measurements of tamper velocity (momentum) were within ter. percent only when the lead and confining walls were in close contact. The observed velocities for even very small gaps were substantially below predictions. These differences are attributed primarily to separation of the liquid and vapor phases during the expansion. 


\section{INTRODUCTION}

There is considerable interest in very rapid (pulse) heating of structural materials. Research efforts in fusion reactor technology ${ }^{1}$, nuclear weapon effects ${ }^{2}$, electron beam welding ${ }^{3}$, and experimental techniques in mining and rock quarrying ${ }^{4}$ are currently attempting to interpret the dynamics of the phenomena involved. Laser fusion reactors must capture the energy released by the nuclear fusion of deuterium and tritium which takes place in nanoseconds and produces terrawatt power densities. The first wall of the reactor must be able to withstand intense and virtually instantaneous heating as of ten as once per second. Nuclear weapons produce energy in the same general time interval as fueion reactors. In an exo-atmospheric exposure or a very close detonation, most of the energy from the explosion will be radiation which will heat the structural components of a target. Depending on range and yield of the weapons, the energy deposition can cause mild heating of the target or it may totally vaporize the target. Hence, designing structures to survive this environment requires a thorough understanding of the effects of rapid heating.

The particular area of interest in this study was the response of a material pulse heated into the tro phase liquid-vapor state. It is much more difficult to predict the material behavior when two phases are present, and repeatable experimental techniques for investigating this region are needed. Depending on the type of radiation, the damage to adjacent layars can vary greatly. One layer may be totally vaporized, 
while its neighbor is still structurally intact, and will thus restrict expension. This "tamping" effect can increase the pressure of the vaporized material and lengthen the time that it affects the other structural layers. The initial layer spacing, materials, and presence of gaps all influence the behavior of the structure.

The first objective of this project was the development of a laboratory technique for measuring the response of tamped expansion of a liquid-vapor mixture. Possible energy sources considered are lasers ${ }^{5}$. pulsed electron beams ${ }^{6}$, and resistance heating devices ${ }^{7}$. Lasers are attractive because of the ease of operation and because any optically transparent material could be used as a tamper. Laser beams, however, will not penetrate the opaque target layer and provide a uniform deposition in a layer which is thick enough to produce the desired amount of liquid-vapor material. Resistance heating techniques have the advantage of generating the energy in the target material making it possible to use any tamping material, but due to circuit inductance, the pulse is orders of magnitude longer than the desired 10-50 nanoseconds. The pulsed electron beam machine was therefore chosen for the energy source because of its suitability and availability for use. The specif ic machine used supplied a 600 joule pulse of $1.0 \mathrm{MeV}$ electrons in 50 nanoseconds. This electron beam had the desired deposition $t$ ime and energy penetration, but it was difficult to produce repeatedly the desired energy levels and distributions. The total energy deposited varied approximately $\pm 20 \%$ from pulse to pulse. 
Lead was selected as the most suitable target material because of its availability, handling, and thermodynamic properties. other materials considered were cadmium (too toxic as a dust and vapor) and uranium (energy required for 50\% vaporization is too great for the available pulsed e-beam source). More powerfil electron beams could be used to study uranium, copper, gold, silver and others. The requirement that the tamper remain sulid led to considering titanium, iron, beryllium, boron nitride, and graphite as tamper materials. Beryllium is too toxic, and boron nitride and graphite are brittle. Since titanium requires more energy to melt than iron, and was available in thin sheets, it was selected as the tamper layer. At higher energy levels the titanium and iron would melt which would leave beryllium as the only choice for the tamper material.

The basic configuration of the test system developed is shown in Figure 1. A matrix of 12 experiment conditions with variable tamper thickness, lead target thickness, and initial cavity size spanned the energy region obtainable with this pulsed electron beam machine. Carbon interface stress gages monitored the stress waves generated in the lead and propagated back into the test fixture. Sub-kilobar and 50 nanoseconds resolution of the stress histories were obtained. A rotating-mirror framing camera took tamper profile photographs at two microsecond intervals. From this data it was possible to establish the tamper velocity. 


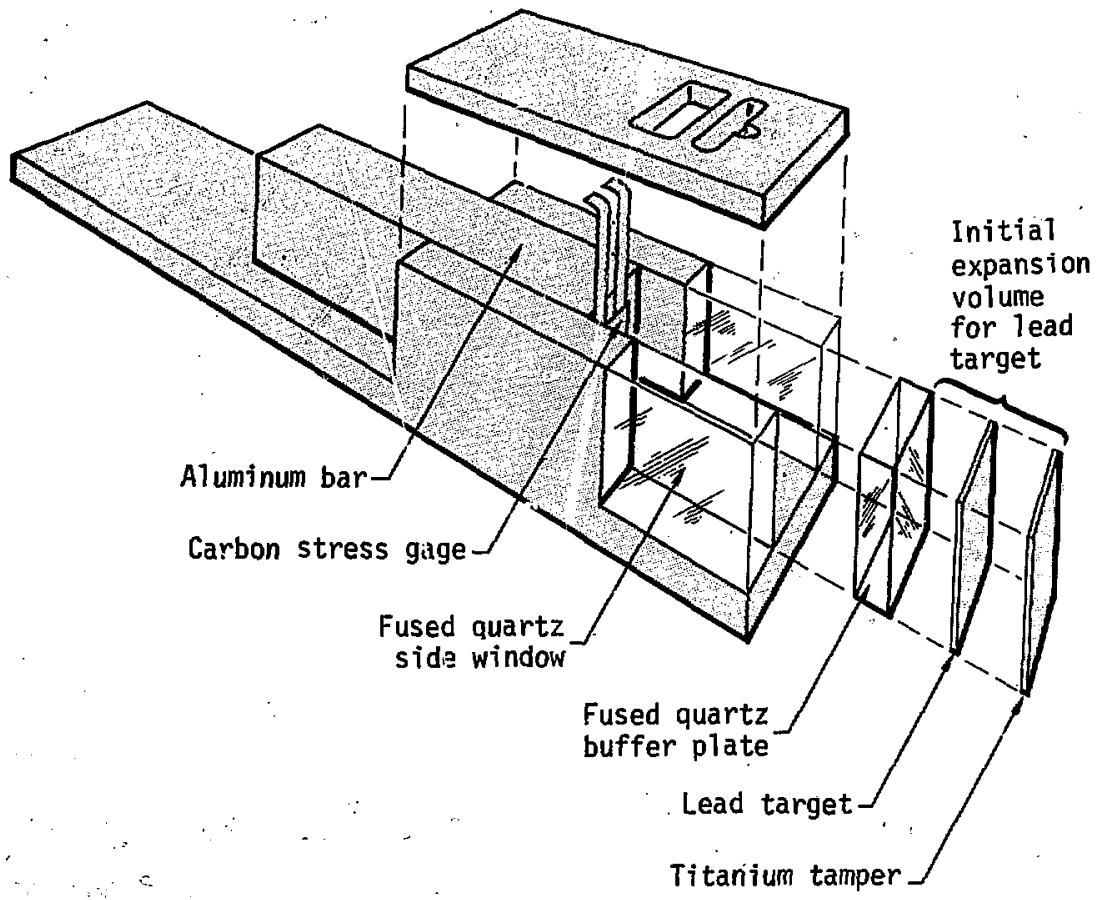

Figure 1. - Schematic diagram of experimental apparatus used for the pulse heating tests. The energy pulse expladed the lead sample causing a measurable stress in the fused quartz plate and an observable motion in the titanium tamper. 
Since a major part of this study was devoted to obtaining reliable experimental measurements, the report was organized to reflect this objective. The sequence of sections is as follows:

\section{Development of Experimental Apparatus}

This section is a detailed account of the necessary steps required to set up the electron beam source and to abtain valid diagnostic information. Over 260 pulses were $f$ ired in this phase.

\section{Laboratory Procedure}

This section describes the procedure for making an experimental test. It covers the assembly of lead and titanium in the target fixture, and the monitoring of electron beam variables to establish the validity of the data run.

Results and Interpretation of Experimental Data

The data are presented with comments on accuracy and repeatability. Post-shot reduction of the framing camera data is explained.

\section{Results of Analytical Studies}

The KOVSPALL computer program with the GRAY equation of state (EOS) provided a means for modeling the experiment. These results are presented in this section along with an analysis of two-dimensional tamper motion using finite element structural analysis.

Discussion of the Results

This secticn is commentary on the comparison of experimental and analytical results. Problems with both areas are discussed.

\section{Conclusions and Recomendations}

Some of the conclusions that can be drawn from the data and analysis are presented. Suggestions $100^{\circ}$ future experiments and applications are made. 
A description of the dynamic response of the pulsed electron beam machine in terms of a mechanical analog is presented. Also included is a brief summary of the computer programs used in data reduction and interpretation.

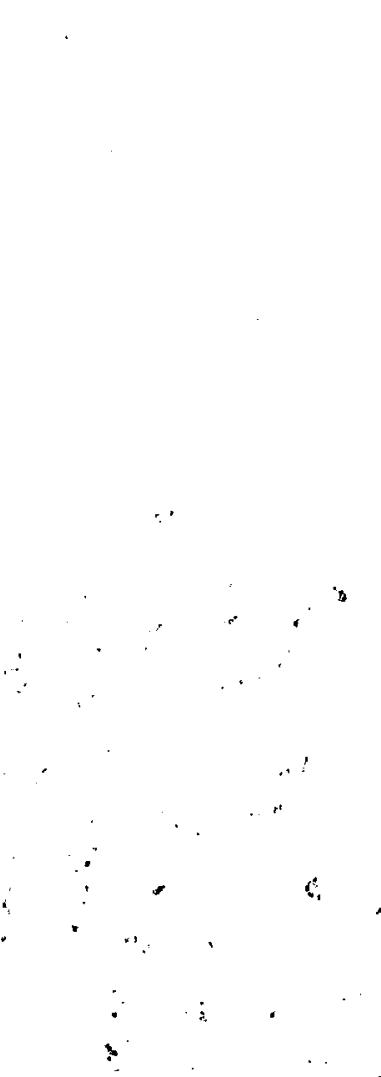




\section{EXPERIMENTAL APPARATUS}

A wide variety of experimental techniques and equipment were utilized in carrying out this study. The principal laboratory facility was a pulsed electron beam (e-beam) which supplied the energy pulse. A large part of the total effort was devoted to establishing the optimum ope, ating conditions for the e-beam machine and this effort will be described in the first two portions of this section.

The balance of the experimental apparatus was the instrumentation and data analys is equipment necessary to characterize the lead specimen and tamper behavior. The major instrumentation systems were: 1) the calorimeters for evaluating the e-beam performance, 2) carbon strcss gages, and 3) a high speed rotating-mirror framing camera capable of taking succesive pictures at two microsecond intervals. Later parts of this section will describe the instrumentation systems and their integration into a unified tesing fixiure.

PHYSICAL DESCRIPTION OF THE ELECTRON BEAM APPARATUS

The energy source for these experiments was a Physics International Pulserad 422 pulsed electron beam machine! It consists of a section housing electrical capacitors and a pulse shaping transmission line.

t - Reference to a company or product name does not imply approval or recommendation of the product by the University of California or the U.S. Department of Energy to the exclusion of others that may be suitable. 
The electron beam machine is ahown in Figure 2 and a plan view of the facility is presented in Figure 3.

The e-beam machine stores up to $25: k i l o j o u l e s$ of energy in capacitors located at one end of the unit. The capacitors are configured as a Marx generator so that they may be charged in a parallel circuit and discharged in series. The stored energy is converted into kinetic energy in a short burst of high velocity electrons with a special transmission line connected to a diade. The electron burst is produced by field emission from a cald cathodi in the diode and the electrons are accelerated towrird a thin plate anode. Most of the electrons pass through the thin anode and are available for use in the experiment. A schematic view of the experiment chamber is shown in Figure 4. For maximum transfer of energy from the transmission line to the diode, the line and diode must have matched impedance characteristics. (Appendix B. contains a detailed treatment of the e-beam circuit response.) To meet this requirement over a range of diode configurations, the transmission line is four feet in diameter and may be either co-axial or tri-axial.

The machine produces an electron pulse appraximately 50 nanoseconds long. The mean energy of the beam can be varied from $250 \mathrm{keV}$ to 2.5 $\mathrm{MeV}$, but the most efficient operating range is from one to two MeV. Circuit conditions required to obtain mean energies below one MeV and above two MeV result in poor impedance matching between the transmission 


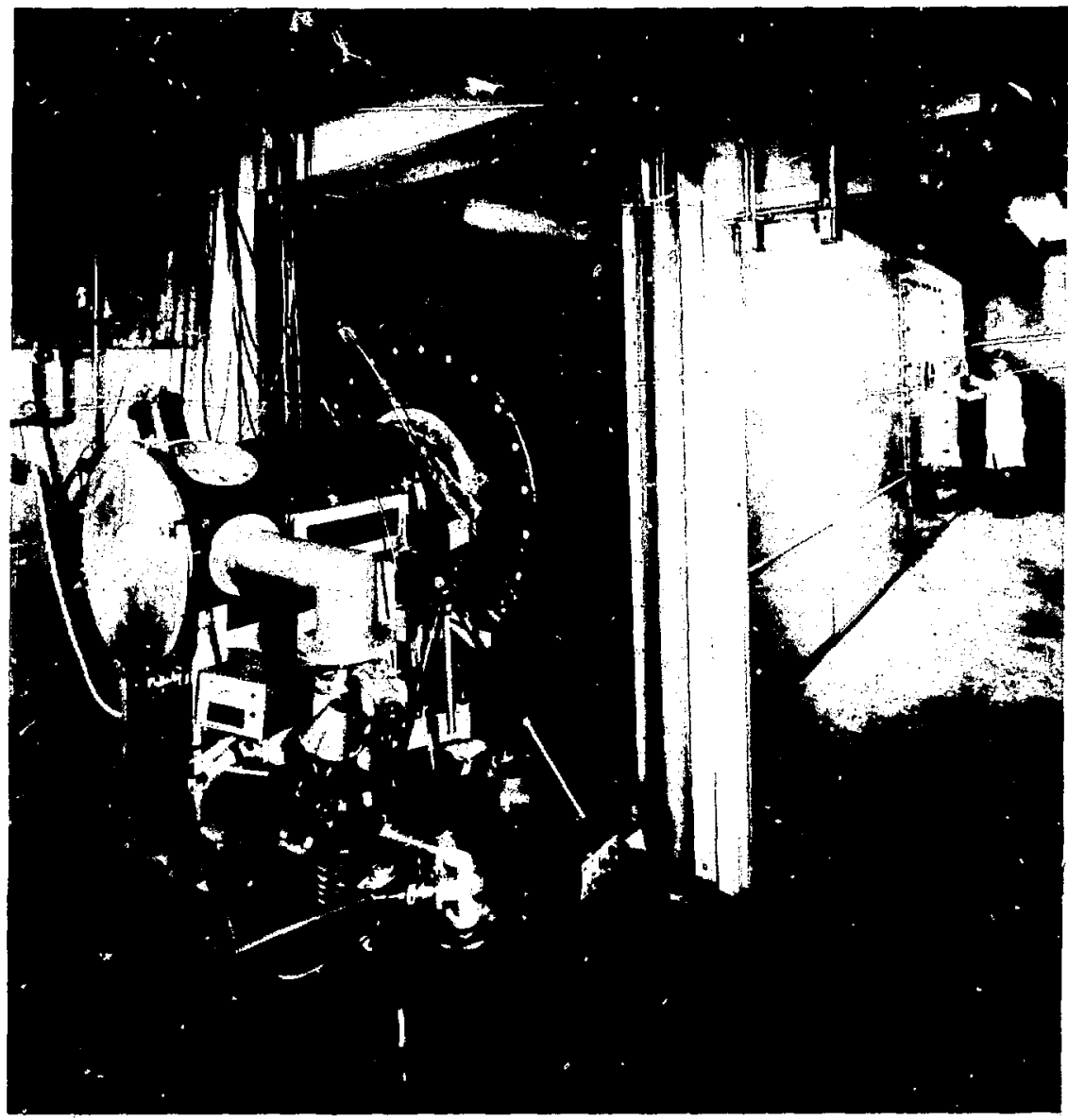

Figure 2. - The pulsed electron beam machine showing the experiment chamber in the foreground and the entry door to the Marx generator section at the right. 
Test cell

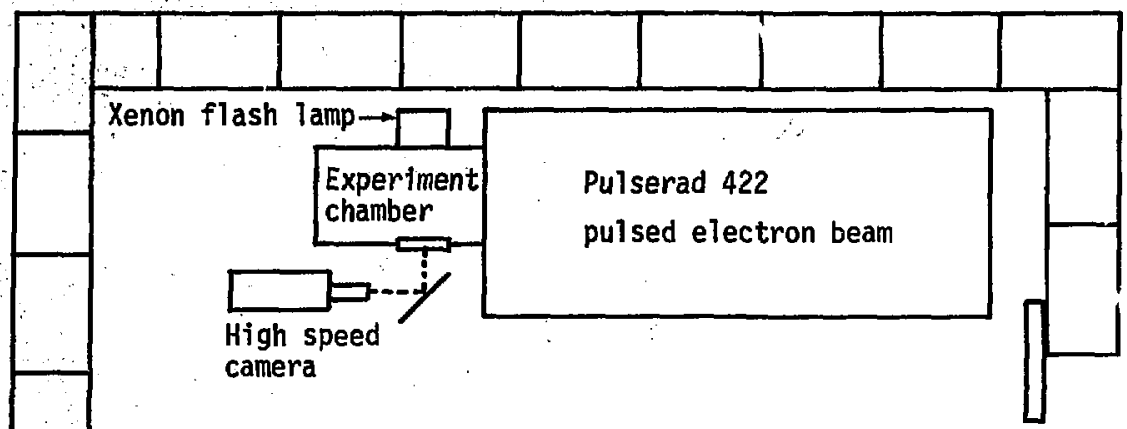

Concrete shielding blocks

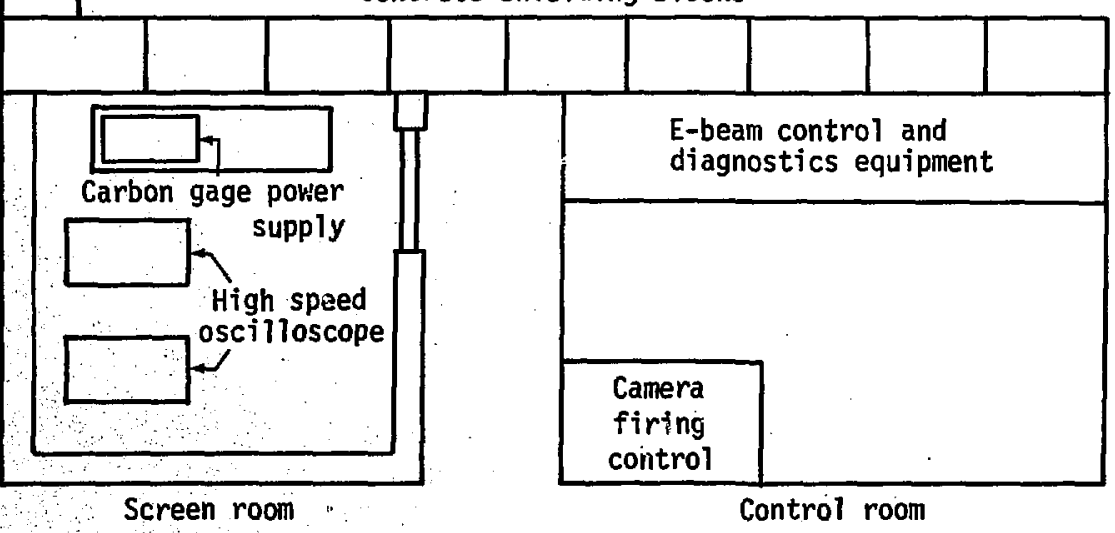

Figure 3. - Plan view of the electron beam facility showing relative locations of the control room, the pulsed electron beam machine, and the diagnost ic equipment. 


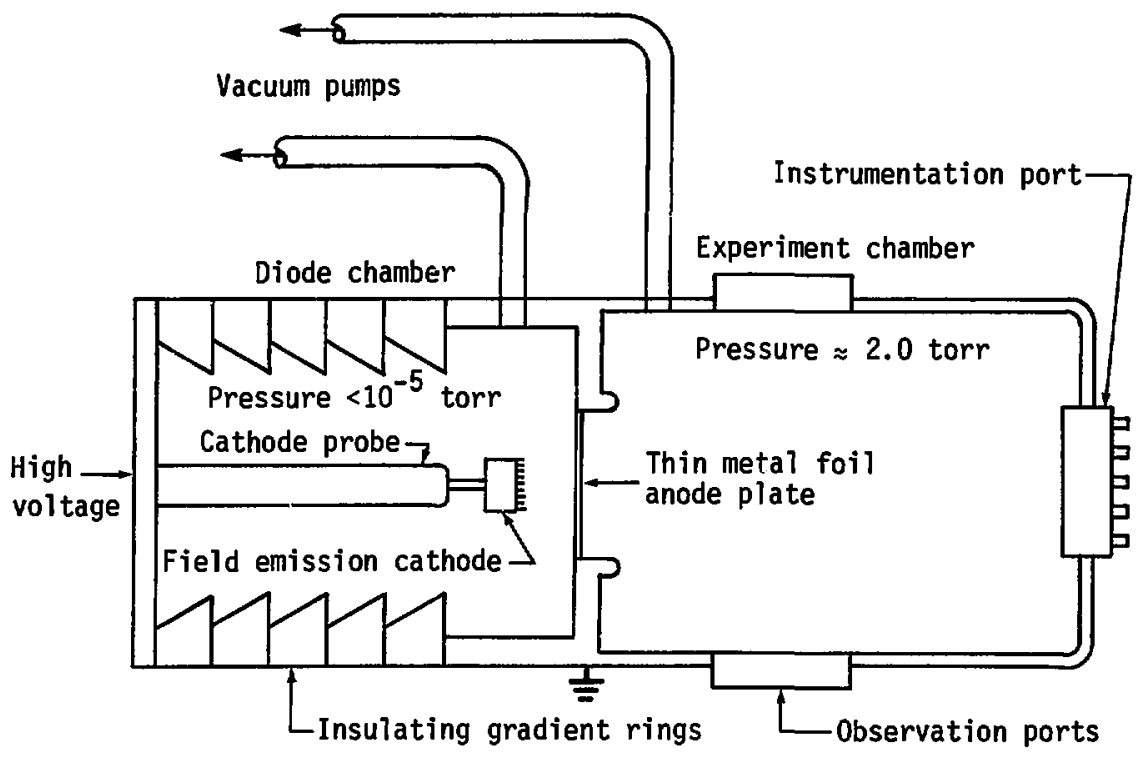

Figure 4. - A schematic diagram of the diode and experiment chambers. The shaped high voltage pulse is applied to the cathode probe. Field emission electrons are accelerated across the cathode-anode space and through the thin foil into the experiment chamber. 
line and diode ${ }^{B}$. A plot of both theoretical and measured total beam energy versus the mean electron energy of the pulse is shown in Figure 5.

The diode impedance changes with time as the electron pulse is produced resulting in a spectrum of electron energies within the pulse. In order to determine the essential characteristics of the pulse, real-time measurements are made for each shot. A voltage versus time history of the Marx generator "charge" to the transmission line indicates the total energy stored in the tranmission line before it is connected to the diode. The voltage across the diode and the current through the diode are also measured as a function of $t$ ime. Theoretically, this would allow a time resolved spectrum of electron energies to be produced for each pulse. It is very difficult to get correct dymamic calibrations for the voltage and current transducers, so the essential value in these measurements is the indication of machine repeatability. The mean electron energy of the pulse was determined by using calorimeters to measure the energy penetration characteristics of the beam. Each of the "real-time" measurements are recorded on $t$ ime correlated high: speed oscilloscopes equipped with poloroid cameras. Figure 6 shows examples of these pulse diagnostic records.

The beam drift chamber shown in Figure 7 is a two foot diameter by three foot long vacuum tank with viewing and instrumentation ports. The gas mixture and pressure in the drift chamber must be precisely controlled to keep the beam stable after it passes from the diode 


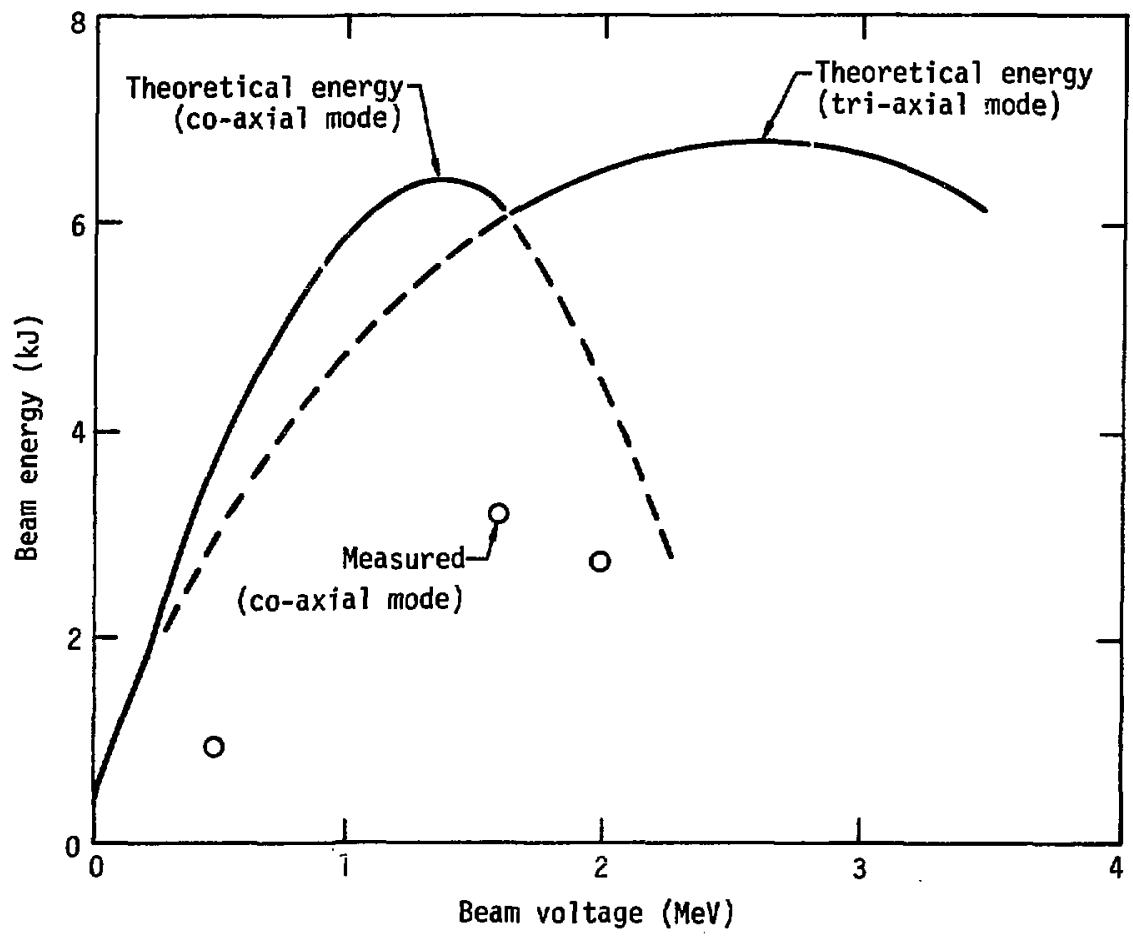

Figure 5. - Predicted and measured total electron energy available in the pulsed electron beam. The maximum output occurs when the diode impedance most closely matches the impedance of the transmission line. (from reference $B$ ) 


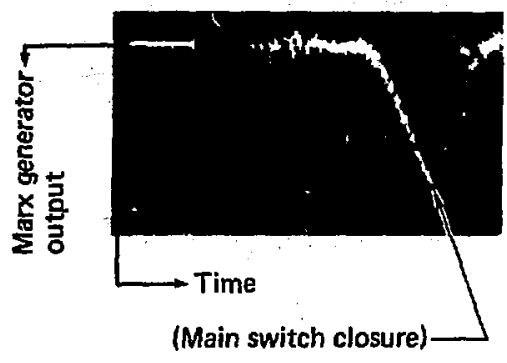

\author{
Pulse charge \\ $610 \mathrm{kV}$ per division \\ 0.2 microseconds per division
}

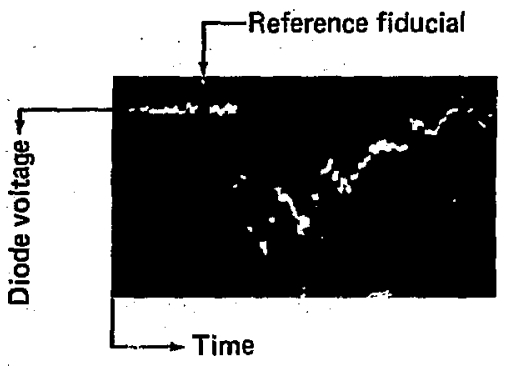

Diode voltage

$361 \mathrm{kV}$ per division

20 nanoseconds per division

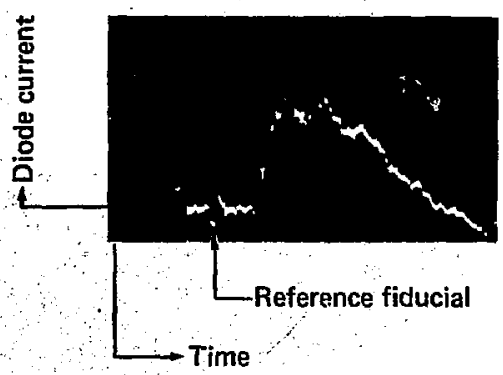

Diode current

24 kA per division

20 nanoseconds per division

Figure 6.- A set of typical electron beam pulse diagnostic records. These measurements of diode performance are useful for monitoring the repeatability of e-beam pulses. 


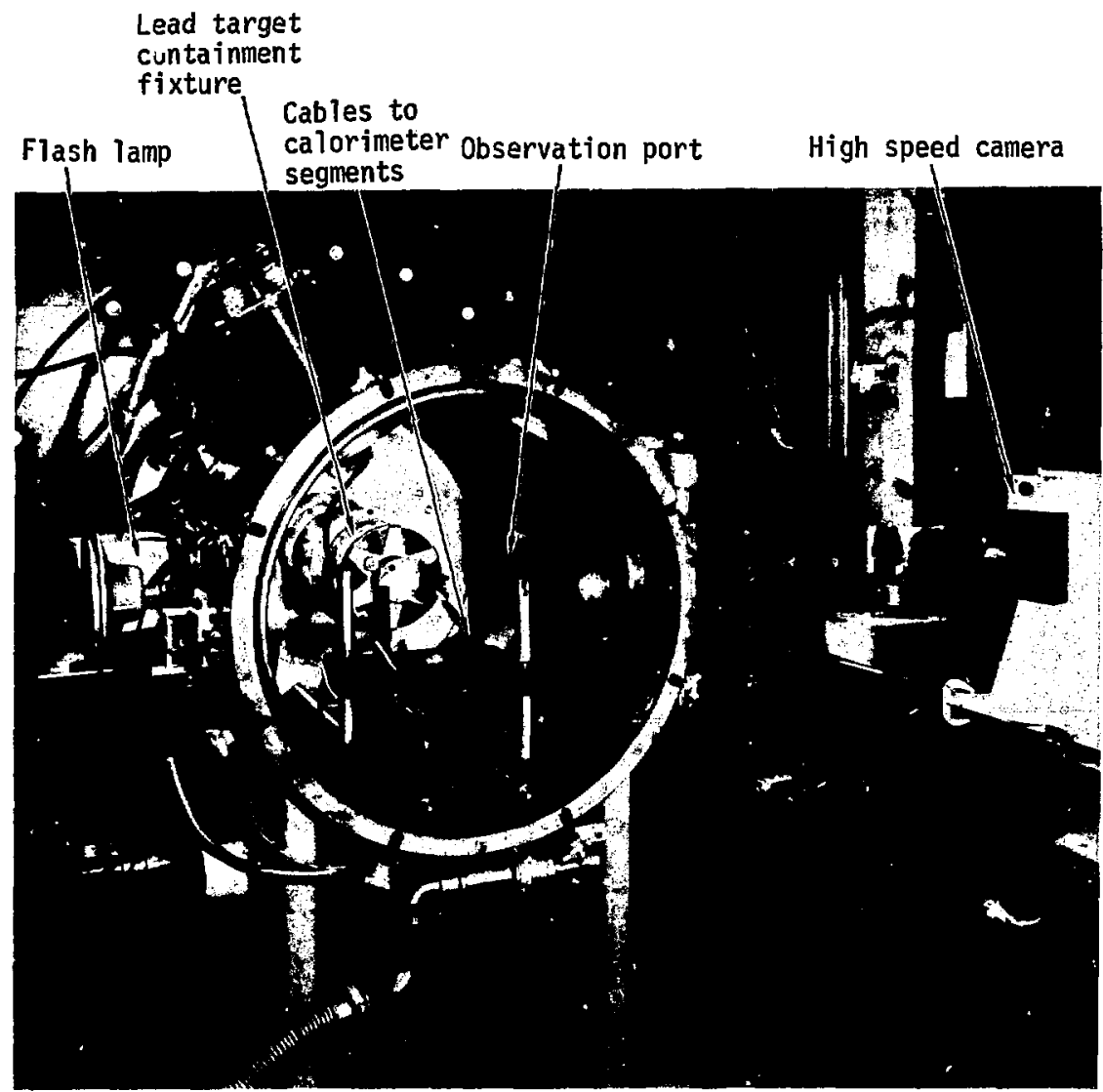

Figure 7. - The experiment (beam drift) chamber with the sample-containing fixture covering the anode. The cable bundle attached to the fixture connects to the thermocouples in the nine segment calorimeter. 
chamber through the anode into the drift chamber. The densily (pressure) of the gas in the drift chamber is used to balance the magnetic and electrostatic forces acting on the pulse as it moves across the drift chamber.

\section{DETERMINATION OF THE OPTIMUM E-BEAM CONFIGURATION}

For this experiment to be successful, enough electrons have to penetrate the tamper layer to heat the lead target into the two-phase region which requires about 160 calories per gram of lead. This involves a trade-off between the electron mean energy and total fluence obtainable. A high mean energy beam penetrates more deeply into the target material and provides a more uniform deposition in the beam direction because the energy deposition gradients are smaller. This also means that the total energy trapped in a given layer is also smaller for an equal amount of onergy incident on the target. Because the energy, deposition per unit depth is smaller at higher mean electron energies, the corresponding total incident fluence must be increased to deposit the same total energy in the fixed thickness target sample. Decreasing the mean electron energy not only makes steeper deposition gradients in the lead target, it also increases the peak deposition in the litanium tamper layer. For a given incident fluence, requiring the tamper to remein solid establishes an allowable lower limit on the mean electron energy. 
A total stopping calorimeter was used to measure the fluence that enters the $3 \mathrm{~cm}^{2}$ cavity and reaches the target plane. This calorimeter trapped all of the electron energy with a subsequent $r$ ise in temperature which could be measured. . The calorimeter was an array of nine carbon blocks, each with a thermocouple embedded near its mass center. The individugl blacks were $5.5 \mathrm{~mm}$ square by $20 \mathrm{~mm}$ long we ighing 1.17 grams . The temperaturé rise associated with a $50^{\circ} \mathrm{cal} / \mathrm{cm}^{2}$ filuence was about $60^{\circ} \mathrm{K}$. The nine blocks evenly divided the target area into a $3 \times 3$ array and each block is thermally isolated from it neighbors. So that the thermocouple output could be easily converted to incident fluence, $a$. computer program called SEGCAl was written to aid in the data reduction phase. A sample of its output is shown in Figure 8 . These fluence maps were made for all calorimetry experinents and the results were used to determine the total fluence of the electron beam; and, with the aid of intermediate filter layers, an estimate of the penetration of the pulse.

The energy deposition profile can be calculated with Monte-Carlo computer techniques. The program used in this work was ETRANMS ${ }^{9}$, which is a Fortran program using Monte-Carlo sampling techniques to calculate electron/photon transport and energy and charge deposition in multi-material one-dimensional slabs. The basis for ETRANMS is the program ETRAN 15, originally developed by Berger and Seltzer ${ }^{10}$. The ETRAN 15 program is capable of following electrons and photons through plane-parallel, single-material targets of finite thickness and tabulating charge deposition and energy deposition within zones, and 
SHOT NO. 7888

$\cup 11 / 22 / 74 \quad 13: 47: 39$

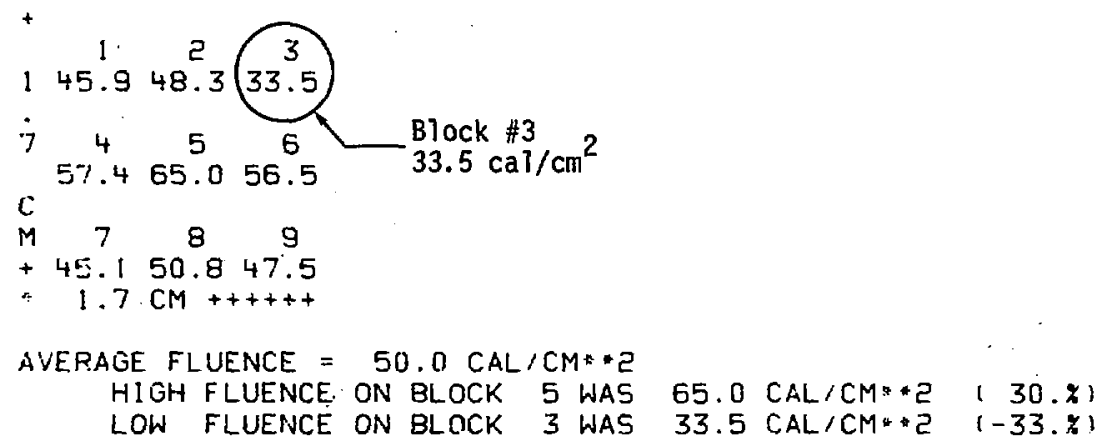

ONE. STO. DEV. $=8.5 \mathrm{CAL} / \mathrm{C}: 4 * 2$

Figure 8. - Teletype output from computer program SEGCAL. The thermocouple data in millivolts for each block is entered and the above data is returned. The nine segment array covers the $3 \mathrm{~cm}^{2}$ target aree. 
electron and photon transport across boundaries. The agreement between ETRANMS and experimental data is considered to be good ${ }^{11}$.

ETRANMS deposition profiles for $0.9,1.0$, and $1.1 \mathrm{MeV}$ mean electron energy deposition in lead are shown in Figure 9 . These are for monoenergetic beams which means that all the electrons incident on the surface of the target are at the same energy level. This is not the case with a high density pulser like the 422 machine which produces a spectrum of electron energies. For the purposes of simulating the energy deposition in the material response calculation, the monoenergetic beam energy that results in a deposition proille that most closely matches that obtained with the actual 422 pulse was used as input to ETRANMS.

The e-beam machine was operated at virtually its peak output with the Marx generator charged to $95 \mathrm{kV}$ and the main switch connecting the pulse shaping transmission line with the diode adjusted for breakdown at 2.5 to $3.0 \mathrm{MV}$. Tests revealed that the energy fraction penetrating through the tamper to the lead target increased as the mean electron energy was adjusted downward from two MeV. This adjustment was done by decreasing the impedance of the diode by closing the gap between the anode and cathode. As the diode and pulse shaper impedances approached a match, the total energy output increased and morc than made up for the loss of penetrating power at he lower mean electron energy. This trend continued until the titanium tamper was at the onset of melt. 
Normalized Energy Density in Lead

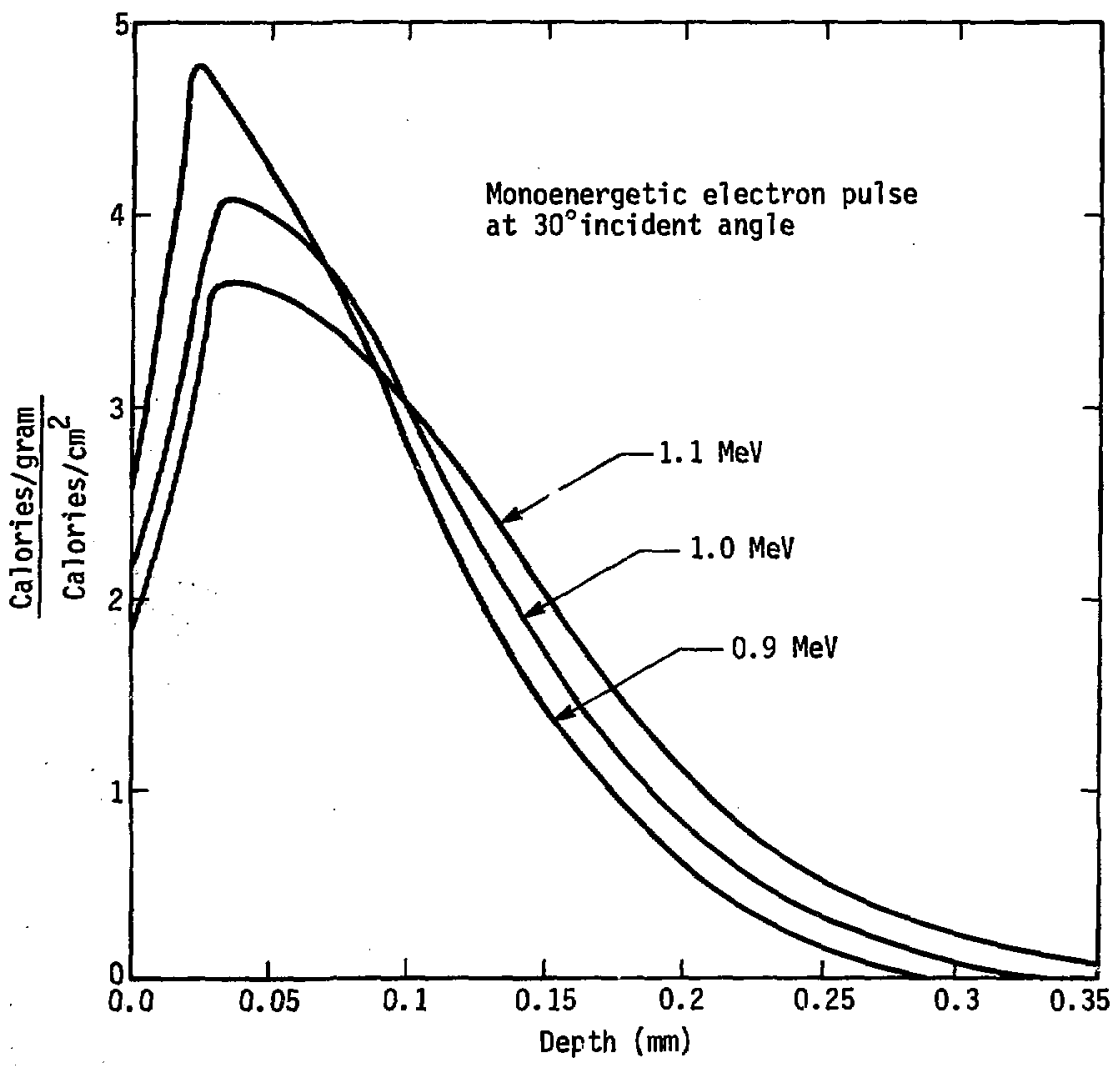

Figure 9. - The energy deposited in cal/gm per cal $/ \mathrm{cm}^{2}$ of incident tluence from several monoenergetic electron beam pulses plotted versus depthof penetration in pure lead. The incident angle is the ang!e at which the electrons strike the target surface as measured from the normal to the surface plane. 
Measurements showed this to be at about $1.0 \mathrm{MeV}$ mean electron energy and $70 \mathrm{cal} / \mathrm{cm}^{2}$ (peak) producing the $280 \mathrm{cal} / \mathrm{gm}$ required to melt titanium.

Figure 10 is a plot of the normalized energy depostion ior one of the experiment configurations. The peak deposition in the tamper was about four cal/gm per $\mathrm{cal} / \mathrm{cm}^{2}$ of ineident fluence. The average energy in the lead layer was approximately $2.75 \mathrm{cal} / \mathrm{gm}$ per $\mathrm{cal} / \mathrm{cm}^{2}$. The deposition in the lead varies plus or minus nearly $20 \%$ from this average value. At $1.0 \mathrm{MeV}$ the mxximum total energy available from the 422 machine averaged over the three square centimeter target area was determined to be $50 \mathrm{cal} / \mathrm{cm}^{2}$. At $1.0 \mathrm{MeV}$ the average deposition in the lead layer was then calculated to be $138 \mathrm{cal} / \mathrm{grn}$. Increasing the mean electron energy allowed fluences greater than $50: \mathrm{al} / \mathrm{cm}^{2}$, but the actual deposition in the lead layer decreased. Below $1.0 \mathrm{MeV}$ the required uniformity of deposition in the lead was not mainta:isd. At $1.0 \mathrm{MeV}$ the output of the electron beam is optimizer .t the co-axial mode.

The net result of ths preliminary testing phase of about 350 experimental shots was a decision that the data experiments weild be conducted with a mean electron energy near $1.0 \mathrm{MeV}$ and at the maximum fluence attainable. This was measured to be between 40 and $60 \mathrm{cal} / \mathrm{cm}^{2}$ averaged over the $3 \mathrm{~cm}^{2}$ target area. If the beam energy became too "cold" (low in mean electron energy), the tamper would show signs of incipient or localized melt. When this occured, a day or two would be devoted to re-establishing the anode-cathode gap required for correct cperation. 


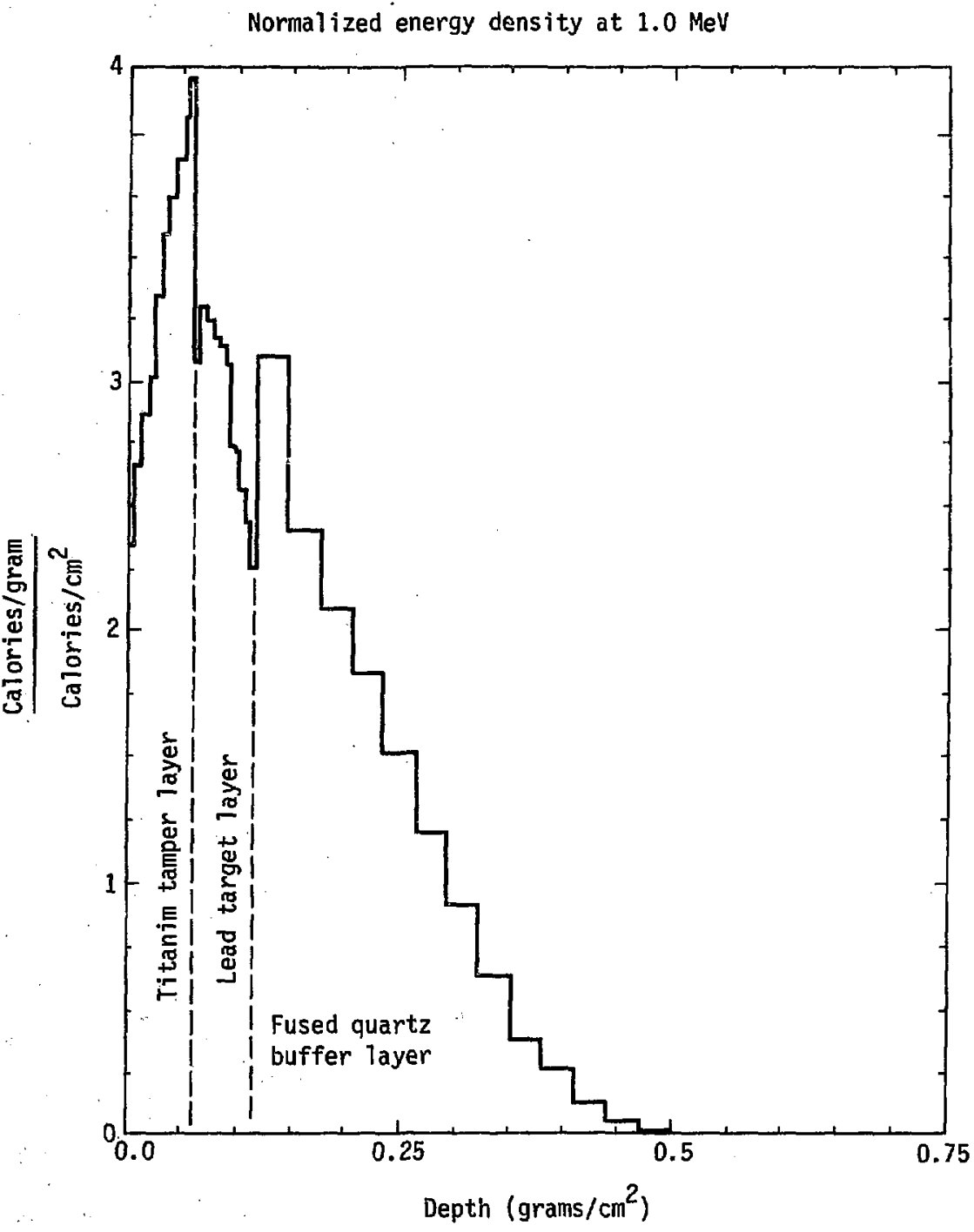

Figure 10. - Normalized energy deposition plotted versus depth for the thin $(0.025 \mathrm{~mm})$ lead target and the thin $(0.125 \mathrm{~mm})$ titanium tamper and a thick quartz buffer plate. Voids are not modeled because they are expected to have a negligable effect on energy deposition. 
The procedure for establishing the mean electron energy was an indirect oria. The direct method of using a stack of thin separated metal foils and measuring the equilibrium temperature of each could not be used at these high energy densities. The foils would melt if made Irom aluminum or tantalum and would be badly distorted by the thermal stresses if they were titanium. Other materials with high specific melting energies like carbon, boron nitride, and beryllium were either too toxic or too fragile to be useful. Even though the material may survive the total energy deposition, small levels of low melting impurities would be enough to destroy the folls. Therefore, the indirect procedure used here was to compare the measured amount of energy thai penetrated various thicknesses of titanium tamper with the amount predicted by ETRANMS for various electron energies. The tamper thickness for the electron penetration lests was varied between $0.125 \mathrm{~mm}$ and $0.375 \mathrm{~mm}$. By plotting the resultant data (Figure 11) with ETRANMS calculations, the approximate ETRANMS input parameters could be established. ETRANMS calculations were scaled with the incident total fluence and used as jnput in the analytical model of the experiment.

The series of 262 shots over a nine month period that were used to optimize the e-beam machine performance led ta the following final operating settings and configuration: 
Energy trapping in a titanium filter layer

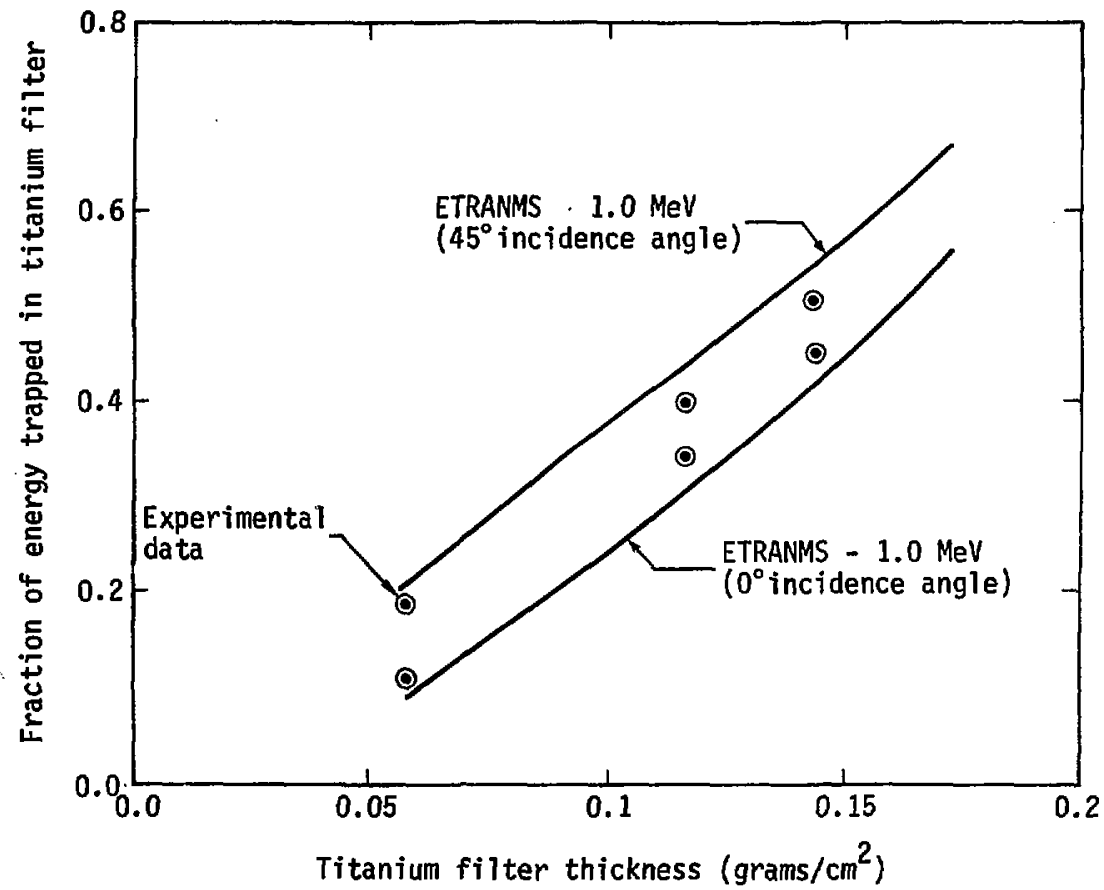

Figure 11. - The fraction of energy trapped in the titanium tamper layer is plotted as a function of tamper thickness. The six experimental points are bounded by ETRANMS predictions using a $1.0 \mathrm{MeV}$ mono-energetic beam at electron incident angles of $0^{\circ}$ and $45^{\circ}$. From this data a 1.0 $\mathrm{MeV}$ beam at $30^{\circ}$ was chosen as the energy source for the KOVSPALL analytical modeling phase. 
1) Capacitor charging voltage $-95 \mathrm{kV}$

2) Main switch gas charge - $130 \mathrm{psi}$ of $\mathrm{SF}_{6}$

3) Cathode $-6.35 \mathrm{~cm}$ diameter rolled pin array

4) Anode - .05 mm aluminum

5) Anode to cathode gap - $17.1 \mathrm{~mm}$

6) Beam guide cone $-6.35 \mathrm{~cm}$ diameter by $30.5 \mathrm{~cm}$ long by

$3.17 \mathrm{~cm}$ dia. solid copper with $3.2 \mathrm{~mm}$ thick walls.

7) Experiment chamber atmosphere - dry $\mathrm{N}_{2}$ at 2.2 tor

The electron beam performance is summarized in Table 1 for these conditions.

Table 1. - Electron beam performance based on the average of the data tests (shots $7800-7921$ ).

Condition Average $(\bar{x})$ Std. dev. $(\sigma) \sigma / \bar{x}$

Maximum charge voltage at switch closure Maximum diode voltage ${ }^{\dagger}$ Maximum diode current ${ }^{\dagger}$ Fluence at target plane

2.2 MV

$1.6 \mathrm{MV}$

$69 \mathrm{kA}$

$50 \mathrm{cal} / \mathrm{cm}^{2}$
$.093 \mathrm{MV}$ $.090 \mathrm{MV}$

1. B kA

10. $\mathrm{cal} / \mathrm{cm}^{2}$
.042

The fluence delivered to the target plane would vary as much as $100 \%$ from one shot to another without a beam guide cone (Figure 12). With a guide cone installed, typical variations were reduced to $10-$ 20\%. The guide cone improves the stability of the electron beam as it flows from the anode to the larget. The rolled pin array cathode (Figure 13) was more effective in providing uniform fluence levels than the flat and hollow carbon plates that were used early in the preliminary phase. The $0.05 \mathrm{~mm}$ luminum anode was the $f$ inal machine variable determined in the preliminary phase. A thinner $0.025 \mathrm{~mm}$ layer t - The calibration of the diode voltage and current traces could not be done dynamically and thus are suspected of having large errors. However, those measurements were very helpful in establishing the repeatability of the e-beam performance from shot to shot. 


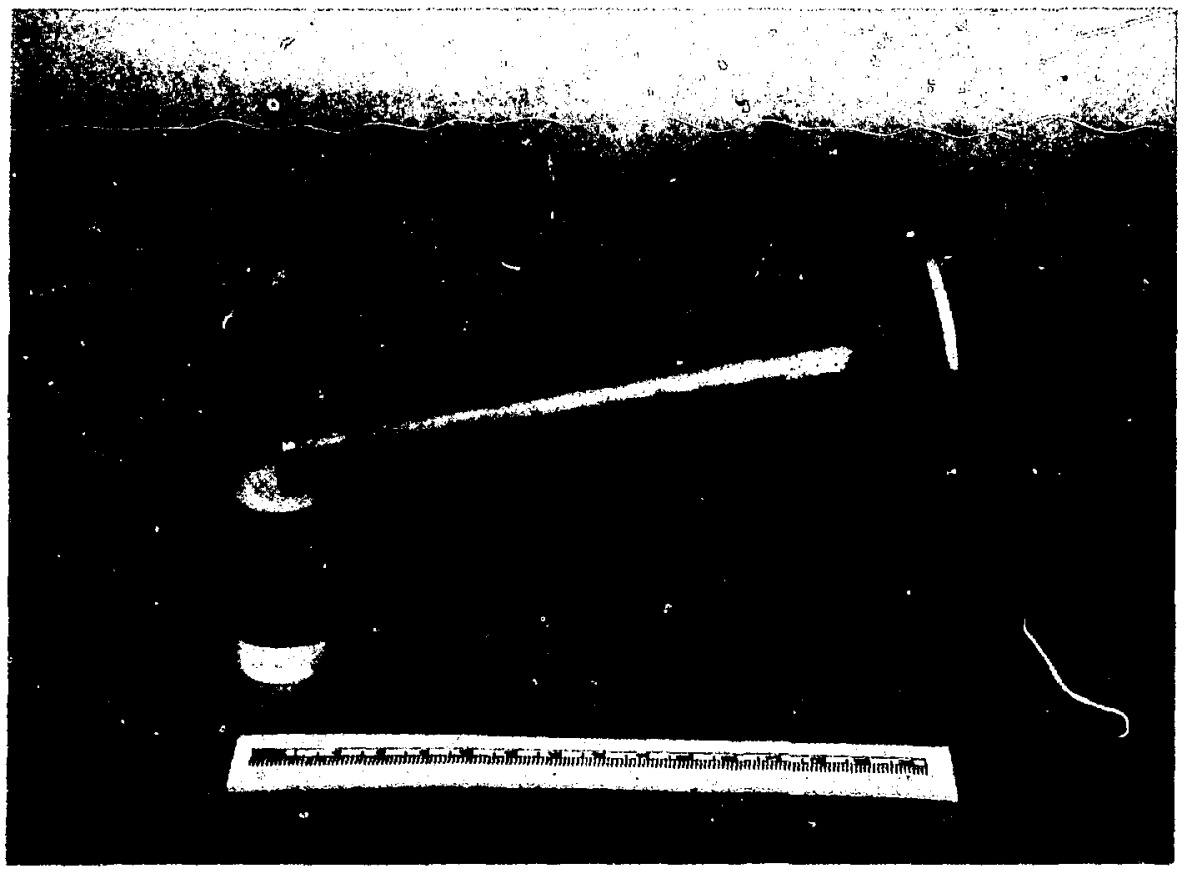

Figure 12. - This electron beam guide cone collected the electron pulse as it emerged from the anode foil and directed it into the aperture in the target holder. 


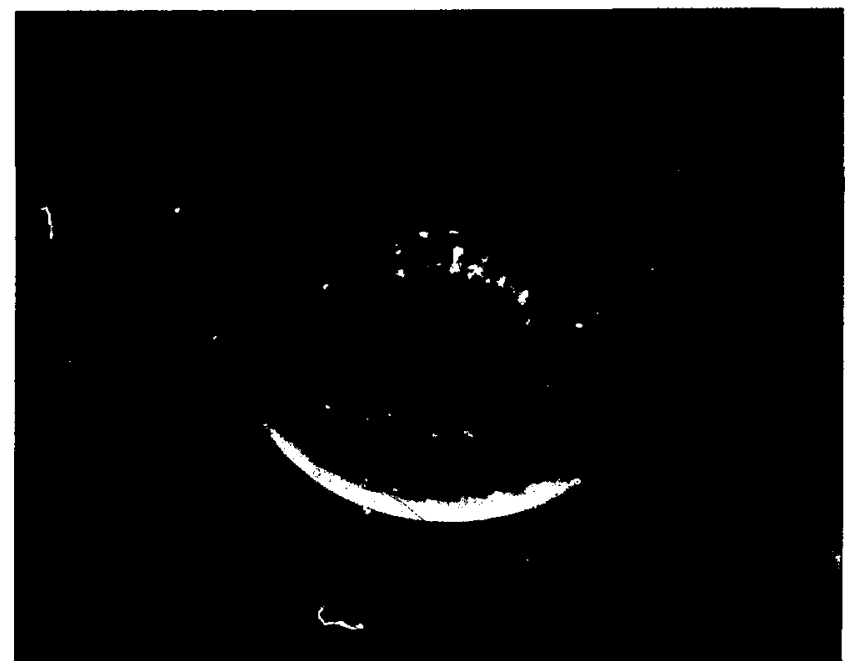

Figure 13. - The field emission cathode was a set of three concentric circles of hollow pins ( $1 / 16$ in. rolled pins) embedded in a brass block. 
of aluminum was tried, but it was found that the greater transmission resulted in melt and general disintigration of the titanium tamper. Conversly, too thick an anode reduced the overall fluence on the test fixture to unacceptable levels.

\section{INSTRUMENTATION AND FIXTURE DEVELOPMENT}

The test fixture was specifically designed to accomodate the two principal measurement systems: the carbon stress gage and the high speed framing camera. It was necessary to place the carbon gage near enough to the expanding lead to measure the stresses generatea by the lead expansion but not so near that the integrity of the gage was lost or that electrical noise was severe enough to obscure the data signal. The walls of the confinement cavity had to be transparent and flat so that an undistorted image of the edge of the tilanium tamper could be recorded photographically. A three square centimeter area was selected for the target to insure that the electron beam would be intense enough to deposit $150 \mathrm{cal} / \mathrm{gram}$ in the lead with acceptable spatial uniformity.

The evolution of an apparatus that would fulfill these requirements resulted in a simple brass fixture which would contain the experiment assembly in a square cavity $17 \mathrm{~mm}$ on a side. The side walls of the cavity were constructed from laboratory grade fused quartz to provide visual access for the framing camera. The top and bottom walls of the cavity were also quartz so that dielectric symmetry was maintained. Preliminary experiments on the need for dielectric symmetry were 
inconclusive, but as an insurance measure, quartz walls were used on all four sides. The walls were replaced whenever they were damaged by energy deposition or by the impact of the expanding lead. This fixture had a contact terminal strip for attaching the carbon gage electrical leads to a co-axial cable which transmitted the output signal to a bridge circuit and oscilloscopes. The nine-segment calorimeter was positioned by a similar fixture which did not have the carbon gage cable at tached.

The carbon stress gage was laminated between a fused quartz buffer plate and an aluminum block stress sink. The buffer plate was $6.35 \mathrm{~mm}$ thick and protected the gage from the expanding lead. When thinner buffer plates were used, the electric field near the carbon gage caused the gage power system to malfunction. Fused quartz was selected as the buffer layer material because of its very low Grüneisen parameter. Only $0.3 \mathrm{kilobar}$ of pressure would be developed in the buffer layer by the electron beam energy that passed through the lead target without being absorbed. Since the pressure produced in the quartz arrived at the gage before the signal from the lead, it was important to keep it small. If it was too large, it would destroy the gage or mask the signal generated by the lead stress pulse. The aluminum block was $76 \mathrm{~mm}$ long and provided a sink for the stress wave that passed through the carbon gage. The length was selected so that a reflection of the stress wave transmitted into the aluminum would not return from its free surface until more than 40 microseconds had elapsed. This buffer/gage/sink 
lamination was held in place in the fixture with a set screw which engaged the rear portion of the aluminum block.

The lead foil sample was rolled to the required thickness and cut so that it could be clamped in the holding fixture at the top and bottom walls. After the lead was in place the stress gage assembly was postitioned behind the lead and the tamper attached in front. Two small drops of a quick setting glue attached the tamper to the fused quartz walls and maintained the tamper alignment. The fixture maintained preset gaps between the lead and the buffer and tamper layers as large as $0.5 \mathrm{~mm}$ wide. These gaps were always equal on both sides of the lead layer providing for symmetric expansion of the lead target before impact on the buffer and tamper occurred. The gaps were maintained by aperture plates constructed from ATJ graphite. Small gaps in the range of 0.01 to $0.03 \mathrm{~mm}$ were established by placing the buffer layer and tamper layer as close to the lead as possible without distorting the lead layer.

The fixture had to be designed to provide for quick return of the deposited electrical charge to the e-beam machine ground plane. Failure to provide for the effective "grounding" of the target caused high common mode electrial signals to be generated in the instrumentation system. The instrumentation system and screen room area were electrically isolated from the ground plane and allowed to float with this common mode potential. Although this floating effect was true for low frequencies, the distributed capacitance between the screen room and the "ground" plane were large enough to maintain good coupling at the 
gigaherta frequencies generated by the electron beam pulse. When the entire system could not be decompled and "floated", the solution was to provide the shortest possible time constant for the ground return circuit. This was accomplished by minimizing the inductance in the return path. A low inductance current return path in the $f$ ixture was created by mounting the fixture inside co-axial tubes. The sample holder was placed inside the central tube and the outer tube provided the current return path. A carbon plate stopped all the electron energy that did not enter the target aperture and returned the current to the outer tube. This coaxial configuration can be noted in Figure 14. The return conductor was continued around. the beam guide tube back to the anode plane of the diode. Figure 15 shows the assembled holding fixture and the beam guide section before they are mated and at tached to the e-beam machine. The small rectangular holes in the return conductor and the inner tube are necessary for the photographic obervation of the tamper position. Figure 16 shows the completed fixture assembly ready for installation in the e-beam test chamber. The co-axial return path decreased the magnitude and duration of the electrical noise "spike" generated in the carbon gage electronics by the e-beam pulse. This design should be used whenever active instrumentation systems are used in e-beam experiments. 


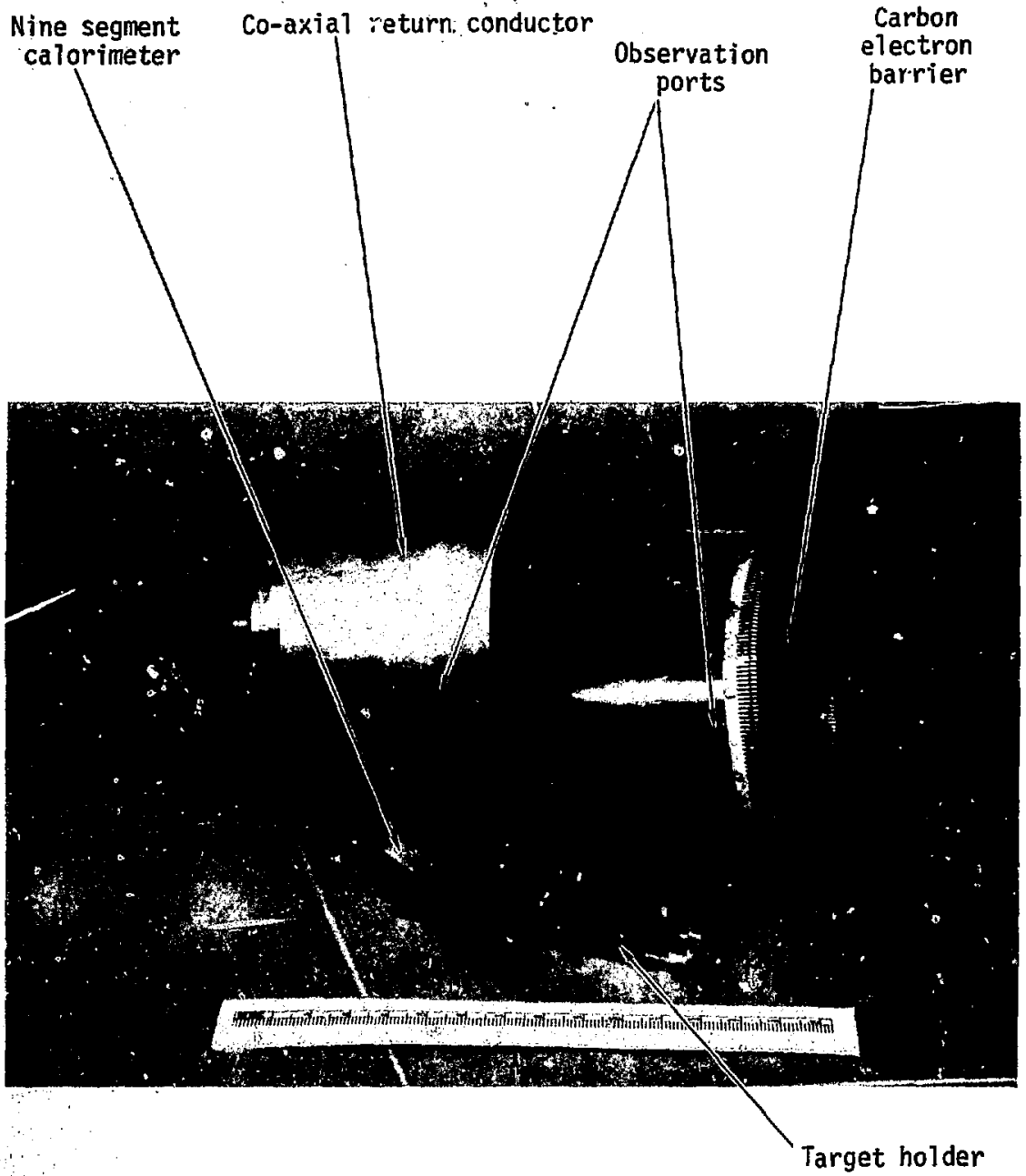

Figure 14. - Test fixture apparatus with the carbon electron barrier shown at the right. All the electrons which do not pass through the aperture and into the lead foil are captured in the barrier. The insertable sample ixture is pictured with the nine-segment calorimeter. 


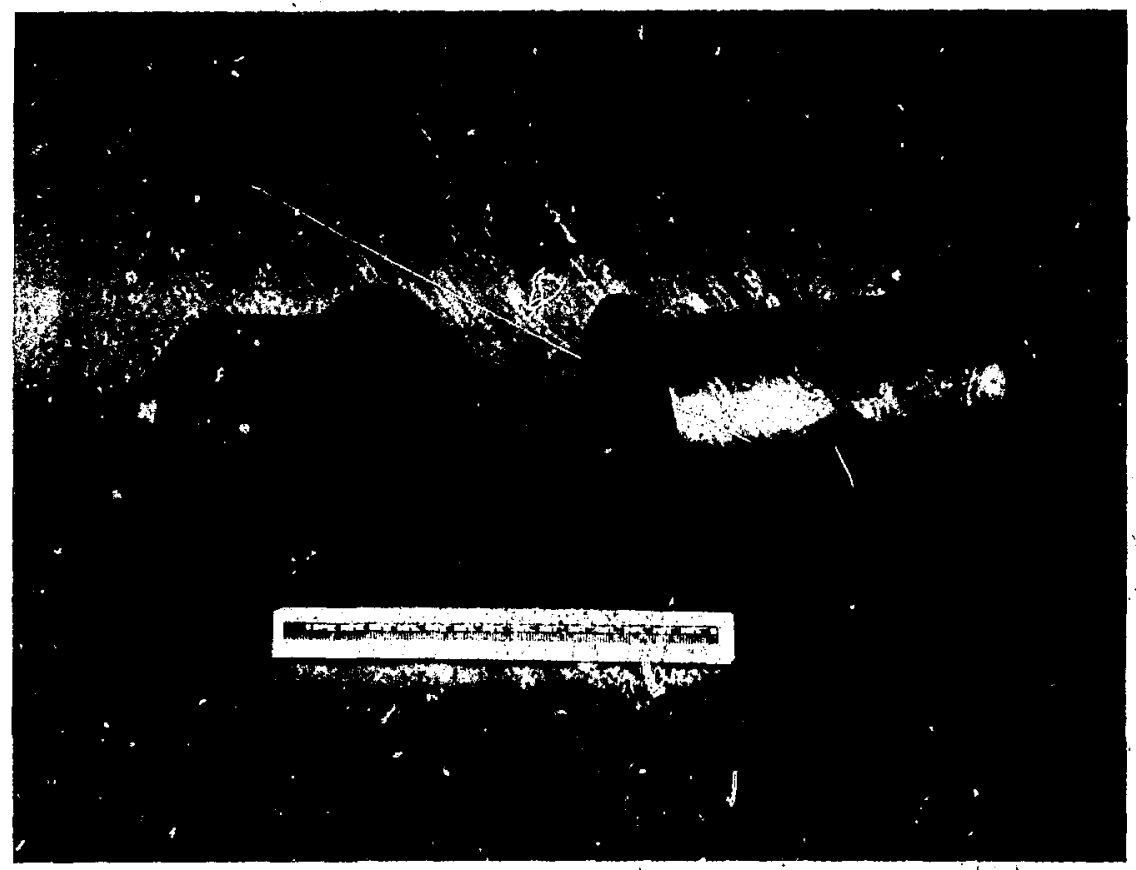

Figure 15. - Test $f$ ixture apparatus showing the beam guide cone at the right. The co-axial configuration provides a pinimum inductance return path for the electrons which reduces the magnitude and width of the noise signal coupled to the carbon gage circuits: 


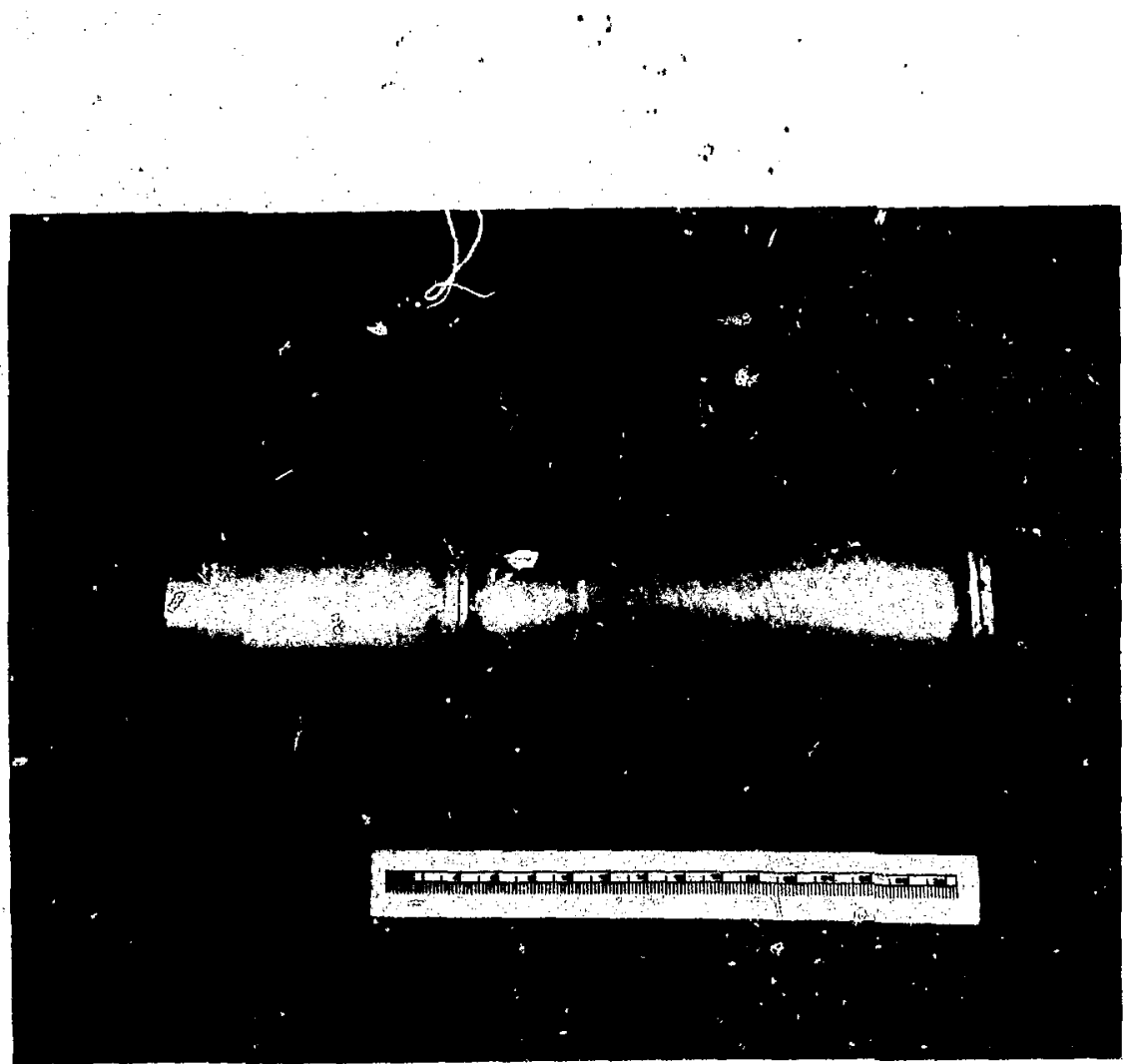

$\therefore$

$\therefore \quad \cdots$

Figure 16. - Fixtureysed to at tach the sample confinement apparatus to the electron beam machine. The electron pulse enters from the $r i g h t$. 


\section{CARBON GAGE PRESSURE TRANSDUCER}

One of the primary measurements in this experiment was the pressure (stress) transmitted to the fused quartz buffer plate. This measurement wạs made with a carbon stress gage ${ }^{12}$ bonded bet,ween the buffer plate and the oluminum block. As noted previously, the aluminum block was included to act as a stress wave absurber and still be a good impedance match to the fused quartz buffer plate. The carbon element in the gage is piezoresistive and the change in resistance caused by an applied stress can be measured with a bridge circuit and recnrded on an oscilloscope equipped with a camera.

The carbon gage (Figure 17) is a small and thin (0.0225 mm) layer of a carbon compound deposited on a suitable backing material. The dimensions of the active element of the gage are such that the nominal electrical impedance of the gage is 50 ohms. Most cables used for high frequency measurement have a characteristic impedance of 50 ohms. For maximum signal transmission and a minimum of reflections the gage must have an identical impedance. The location of the carbon gage installed in the lead target containment fixture is indicated in Figure 18.

The carbon gage backing material is a commercially available polyimide sheet 0.025 mn thick. Foil leads are formed by vapor depositing one micron of gold on the backing and on the edges of the active element. The gold can be covered with a layer of vapor deposited copper to provide a more durable soldering surface. A cover layer of $0.025 \mathrm{~mm}$ polyimide is bonded to the top of the gage providing electrical 


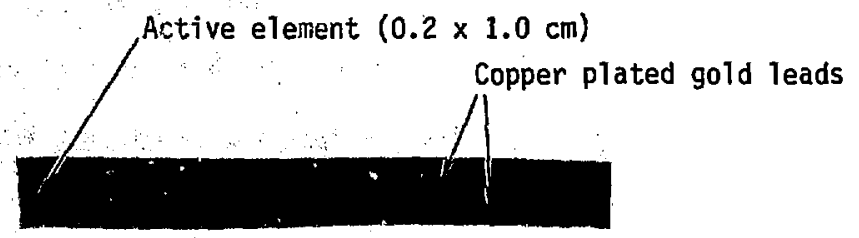

(Actual size)

Figure 17. - The carbon stress gage used for these experiments was encapsulated in layers of polyimide film. The leads are vapor deposited layers of gold with a thin layer of copper added to improve the soldering characteristics. The total thickness of the gage was about $0.075 \mathrm{~mm}$

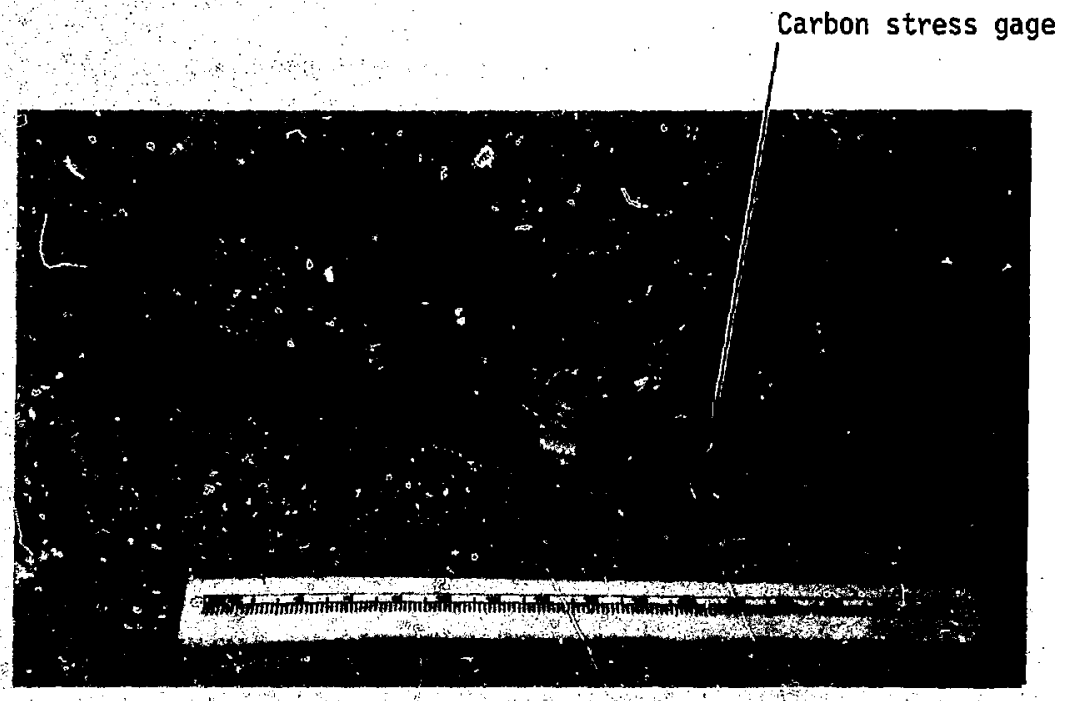

Figure 18 - A detalled view of the insertable test fixture used to hold the lead foil target and the titanium tamper A double shielded co-axial cable was at tached to the carbon gage with the terminal block on the top surface of the fixture. 
isolation of the active element and the leads. This cover was necessary even when the layers of adjacent material were non-conducting (e.g. fused quartz) because the corona from the electron beam would cause the gage exitation voltage to arc across the three millimeter gap between the two leads.

The gage response sensitivity is defined by a gage factor, $k_{a}$, where:

$$
k_{\sigma}=\frac{\left[\begin{array}{c}
\Delta \mathrm{R} \\
\mathrm{R}_{0}
\end{array}\right]}{\sigma} \quad \begin{aligned}
\Delta \mathrm{R} & =\begin{array}{l}
\text { Change in carbon gage resistance. } \\
\text { due to applied stress. }
\end{array} \\
\mathrm{R}_{0} & =\begin{array}{l}
\text { Zero stress resistance of the } \\
\text { gage. (near } 50 \text { ohms) }
\end{array} \\
\sigma & =\text { Magnitude of the applied stress. }
\end{aligned}
$$

The change in resistance of a carbon gage is nonlinear in that the magnitude of the gage factor decreases with increasing compression stress. The sensitivity of the stress gages used in this project is shown in Figure 19. This type of gage is not suitable for measuring tensile stresses because it is limited in tensile strength by the strength of its weakest component layer. The data in Figure 19 show the gage factor is negative when compression stress is considered to be positive. This follows from the fact that the resistance in the gage decreases with compressive stress.

To get a satisfactory signal to noise ratio the power supply ${ }^{13}$ provided a 62.5 volt gage exitation pulse that was 25 microseconds long. The power supply had a capability of providing up to 300 volts for a duration of 100 microseconds. The. Joulean heating in the carbon element 


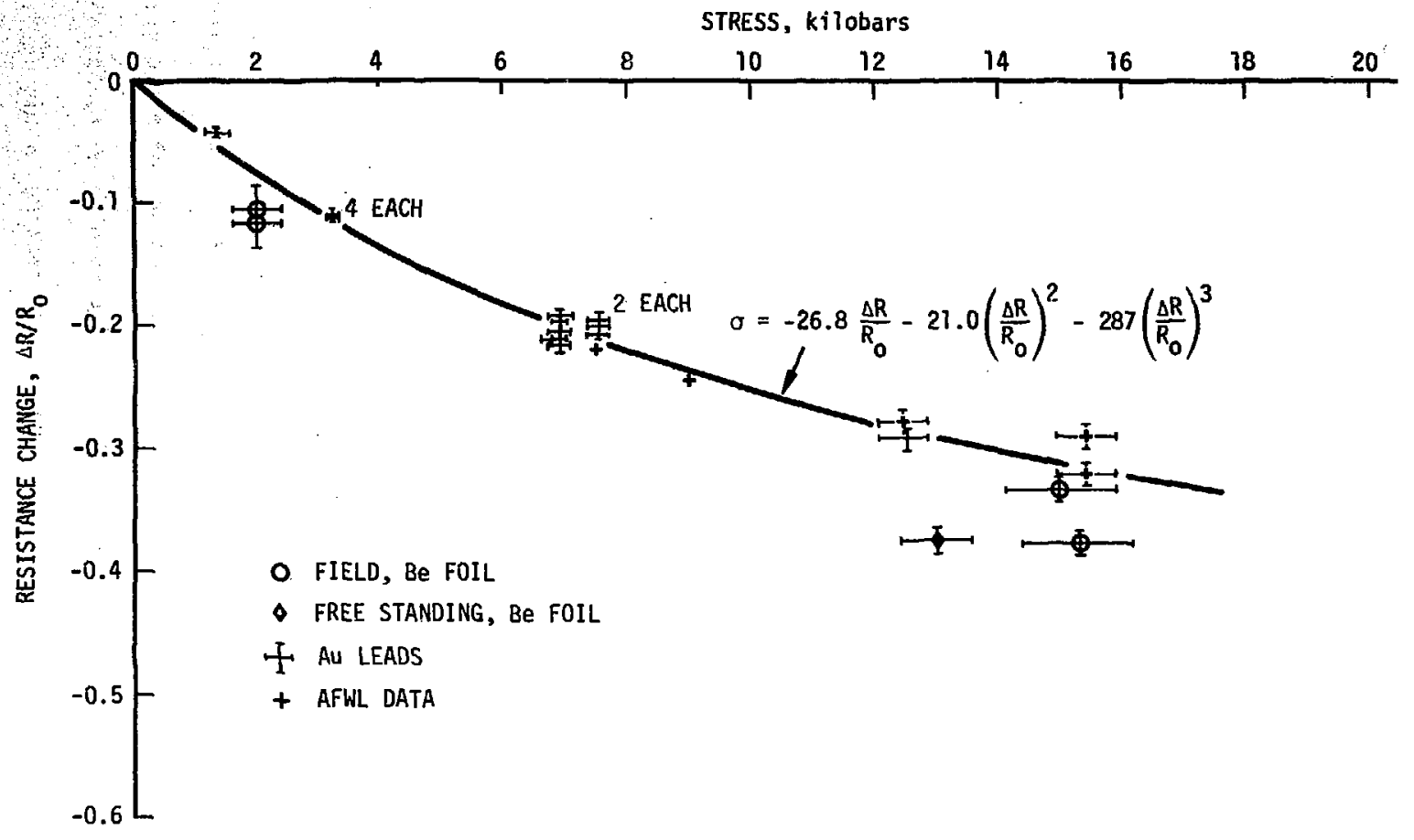

Figure 19. - The carbon stress gage sensitivity to changes in stress in terms of the fraction of resistance change. (from Reference 12) 
produced by a 62.5 volt, 1.25 amp signal produces a negligable resistance change in the gage during the five microseconds of data aquisition. When operated at 62.5 volts, the carbon gage had an output sensitivity of 0.76 volts per kilobar of stress in the gage. The basic power supply circuit as shown in Figure 20 has been designed to elimisate or minimize undesirable reflections in the cable and attenuation that arise from impedance matching prob'ems.

The energy used to supply the bridge is stored on the Iarge capacitor. This capacitor is switched into the bridge five microseconds before firing the electron beam. It takes two to four microseconds for the transient signals to settle out and a steady state condition to be established in the gage circuit. When the electron beam is fired, stress waves which change the resistance of the carbon gage are propagated to the gage by the fused quartz buffer. This resistance change results in a voltage that is propagated as a wave back to the power supply. For an accurate reconstruction of what occurs at the gage end of the cable, there should be no reflections in the bridge circuit. Stated in another way, this requirement specifies that the bridge must $R_{g}$ ohms when viewed from the carbon gage. This constraint is expressed by Equation (2):

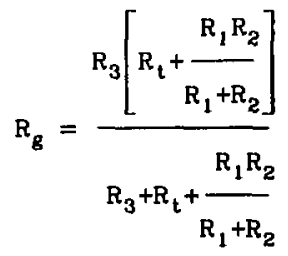




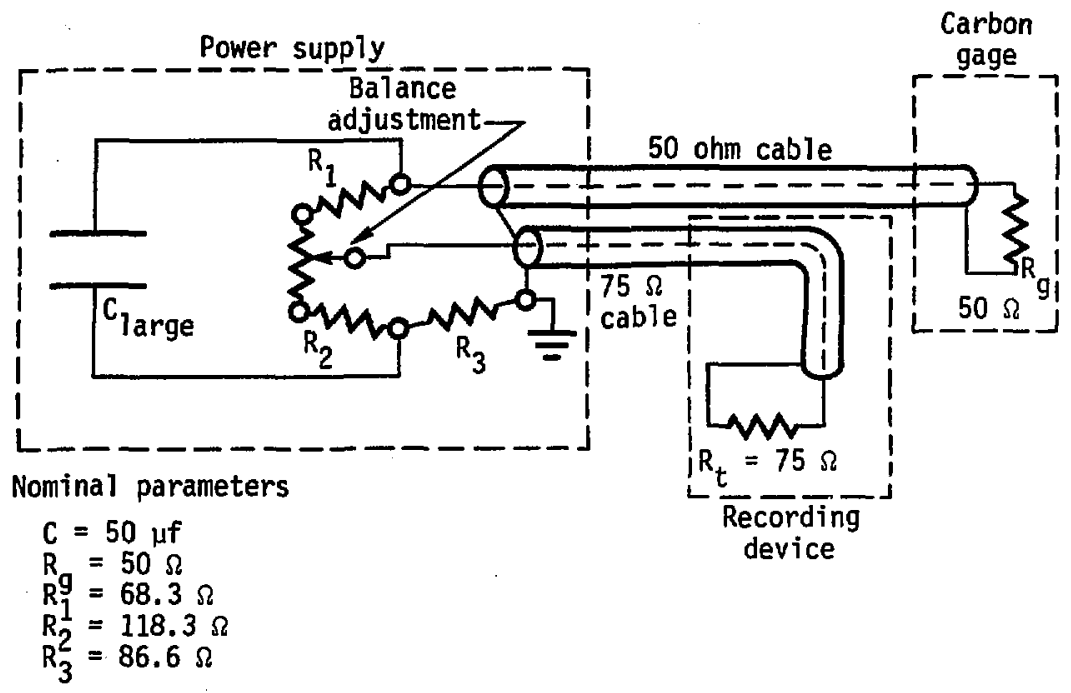

Equivalent circuit:

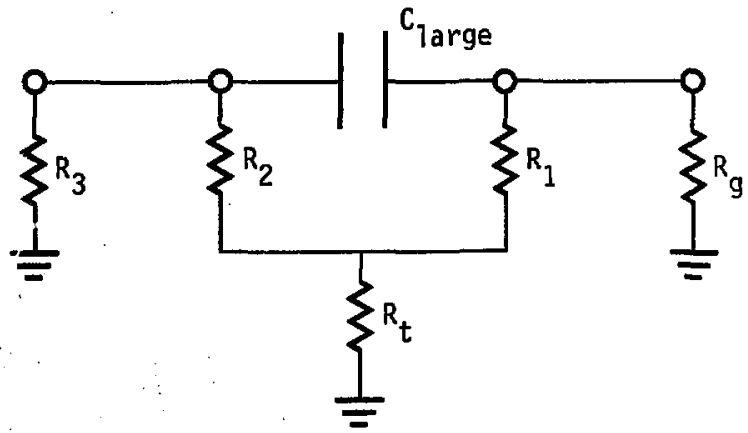

Figure 20. - The electrical circuit for the carbon stress gage system. The capacitor (C) is large enough to be considered a constant voltage source for the duration of the data signal. The resistance of the gage is set to match existing cable impedances and the other values are set so that the impedances are matched at all junctions thereby eliminating signal reflections. 
Note that capacitor C (Figure 20) is large and appears as a short circuit to signals with microsecond or shorter periods. Applying the same "no reflection" constraint to the output portion of the circuit results in Equation (3):

$$
R_{t}=\frac{R_{1} R_{2}}{R_{1}+R_{2}}+\frac{R_{3} R_{B}}{R_{3}+R_{g}}
$$

Equations (2) and (3) can be combined to eliminate $R_{1}$ and $R_{2}$ yielding the following relationship for $R_{3}$.

$$
R_{3}=R_{g} \sqrt{\frac{R_{t}}{R_{t}-R_{g}}}
$$

which places the additional criterion that $R_{t}$ be greater than or qual to $\mathrm{R}_{\mathrm{g}}$. The purpose of the bridge circuit is to make the output signal zero when the gage resistance is unchanged. For this to occur, Equation (5) must be satisfied.

$$
R_{g}=R_{3}\left[\begin{array}{c}
R_{1} \\
- \\
R_{z}
\end{array}\right]
$$

Choosing $R_{g}$ to be 50 ohms and $R_{t}$ lo be 75 ohms and using Equations (2), (3), and (5) results in the following values:

$$
\begin{aligned}
& R_{1}=68.3 \text { ohms } \\
& R_{2}=118.3 \text { ohms } \\
& R_{3}=86.6 \text { ohms }
\end{aligned}
$$

In practice, $R_{1}$ and $R_{2}$ may be adjusted slightly by means of a balance 
potentiometer to provide for zero output from gages that are not exactly 50 ohms at zero stress.

The energy supplying capacitor, $C$, must be charged to a voltage that will produce the desired current in the carbon gage. For these experiments this was 1,25 amps and 62.5 volts across the carbon gage. The necessary potential on tie capacitor is computed from Equation (6)

$$
v_{c}=v_{g}\left[\frac{R_{g}+R_{g}}{R_{g}}\right]
$$

to be 171 volts. The change in voltage at the gage caused by a stress wave is given by Equation (7).

$$
\Delta V_{g}=\Delta R \frac{V_{g}}{R_{g}}=\sigma k_{\sigma} V_{g}
$$

The voltage change $\Delta V_{g}$ is passed through the bridge to the output connector where it is $\Delta V_{t}$, given by:

$$
\Delta V_{t}=\frac{R_{t}}{\frac{R_{1} R_{2}}{R_{1}+R_{2}}+R_{t}} \Delta V_{g}=0.63 \Delta V_{g}
$$

By measuring the initial resistance of the carbon gage and any output bias voltage that exists at shot time, the actual values of $R_{1}$ and $R_{2}$ can be determined. This was accomplished in the stress gage data reduction computer program, STRESSDAT, written for these experiments. The oscilloscope data were encoded with a hand operated point by point 
digitizing device attached to a time shared computer system. STRESSDAT used the cable resistance, and the initial values of $R_{g}$ and $R_{t}$ to calculate the actual values for $R_{1}$ and $R_{2}$. The digitized oscilloscope data were then converted to resistance changes in the gage and the resistance changes to stress using the third order polynomial fit from Figure 19 .

\section{HIGH SPEED CAMERA SYSTEM}

A rotating-mirror framing camera (Figure 21) was used to photograph the postion of the titanium tamper at two microsecond intervals. Up to 30 images (frames) can be placed on stardard $35 \mathrm{~mm}$ cassette $\mathrm{film}$. As shown in Figure 22 the $f \mathrm{ilm}$ is stationary and the jmage is swept irom one frame to the next with a rotating mirror. The mirror rotor is air driven and can be operated at speeds up to 7000 rps providing a inaximum framing rate of $3.4 \times 10^{\circ}$ frames per second. At 7000 rps 30 frames will cover a span of 8.82 microseconds. The image has a primary focus at the slit mirror (see Figure 22) and is again focussed by the intermediate lens to a sharp image on the rotating mirror. Individual lenses relay the image from the rotating mirror to the film plane.

A mechanical shutter will not operate at speeds compatible with the framing rate and thus the "capping shutter" used to keep light out during film loading and set-up is held open for several seconds spanning the experiment event. A high intensity xenon flash lamp is synchronized with the rotating mirror to provide light to the object only when the 


\section{Relay mirror}

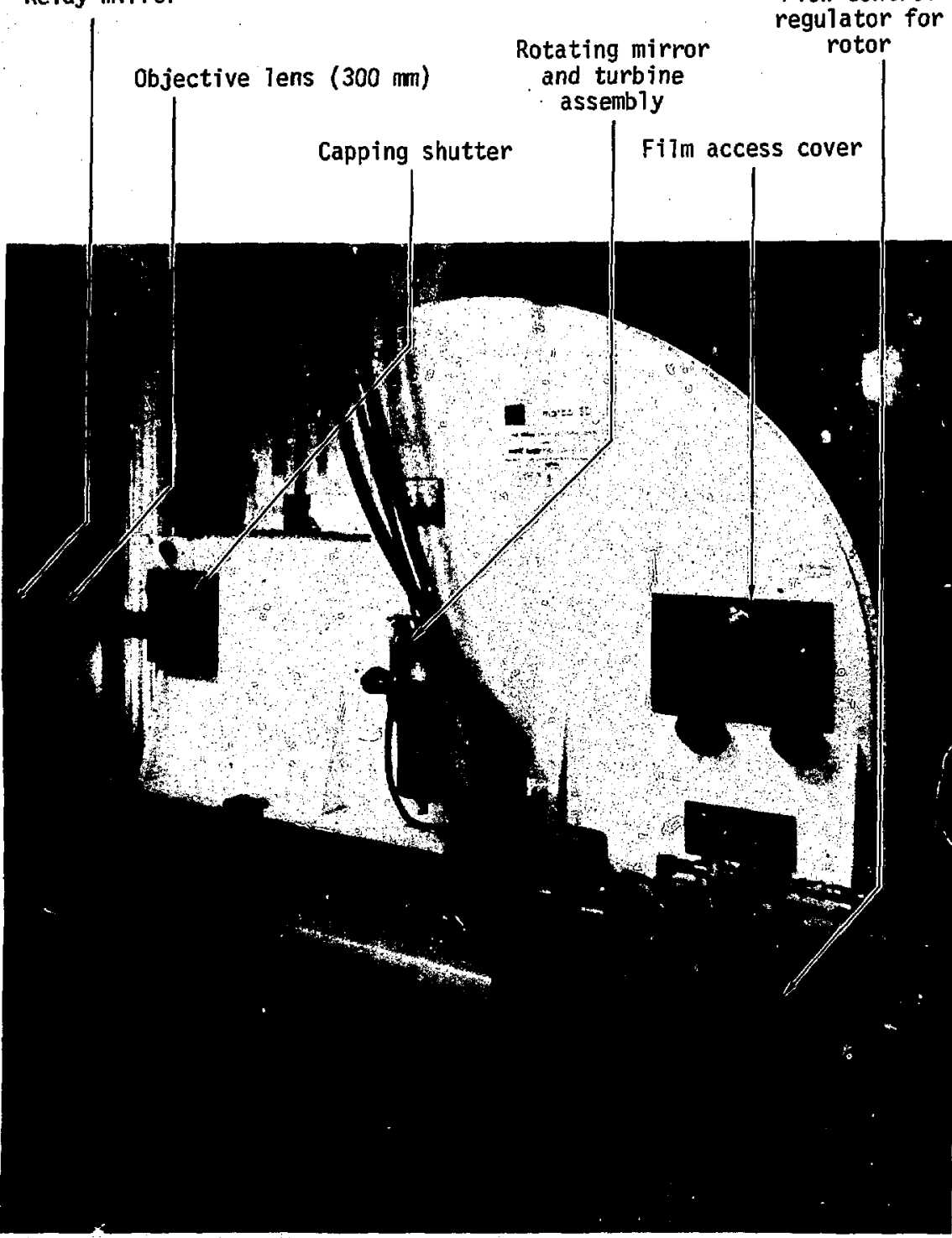

Figure 21. - The high speed rotating mirror camera used to observe the tamper postion as a function of time. The frames were taken at two microtecond intervals. 


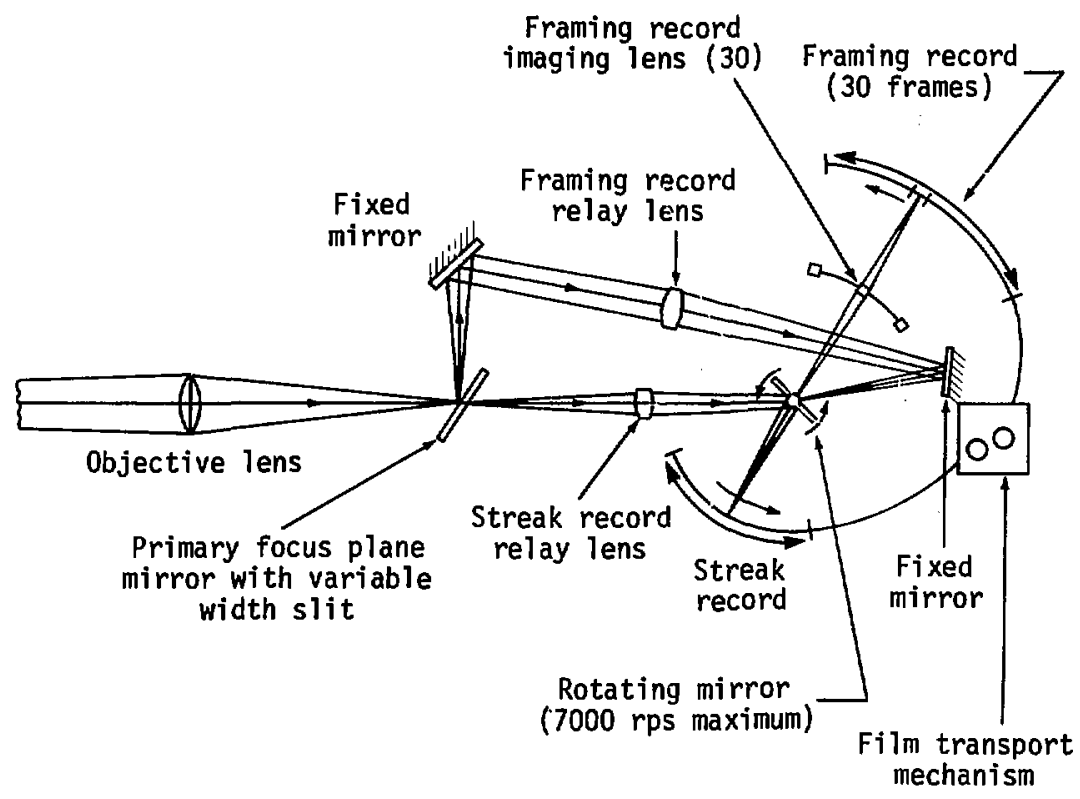

Figure 22. - Schematic diagram of the high speed camera lens system. The $300 \mathrm{~mm}$ objective lens provided a $1 \mathrm{~lm}$ image $\frac{1}{4}$ actual size. (This camera will simultaneously make a "streaking" record using the slit aperture in the primary mirror. This feature was not used in these tests.) 
Irame images are being recorded. Because the rotor cannot be synchronized, all the experiment timing signals must be initiated by the rotor. When the rotor reaches the proper speed and various interlocks are closed, the camera control circuitry issues a trigger pulse nominally 20 microseconds before the first frame is recorded. This signal is used to sequence events such as flashing the xenon lamp. initiating the e-beam pulse, and pre-triggering the recording systems to start monitor channels and energize the carbon gage bridge circuit. These functions are outlined in the line diagram of Figure 23. 


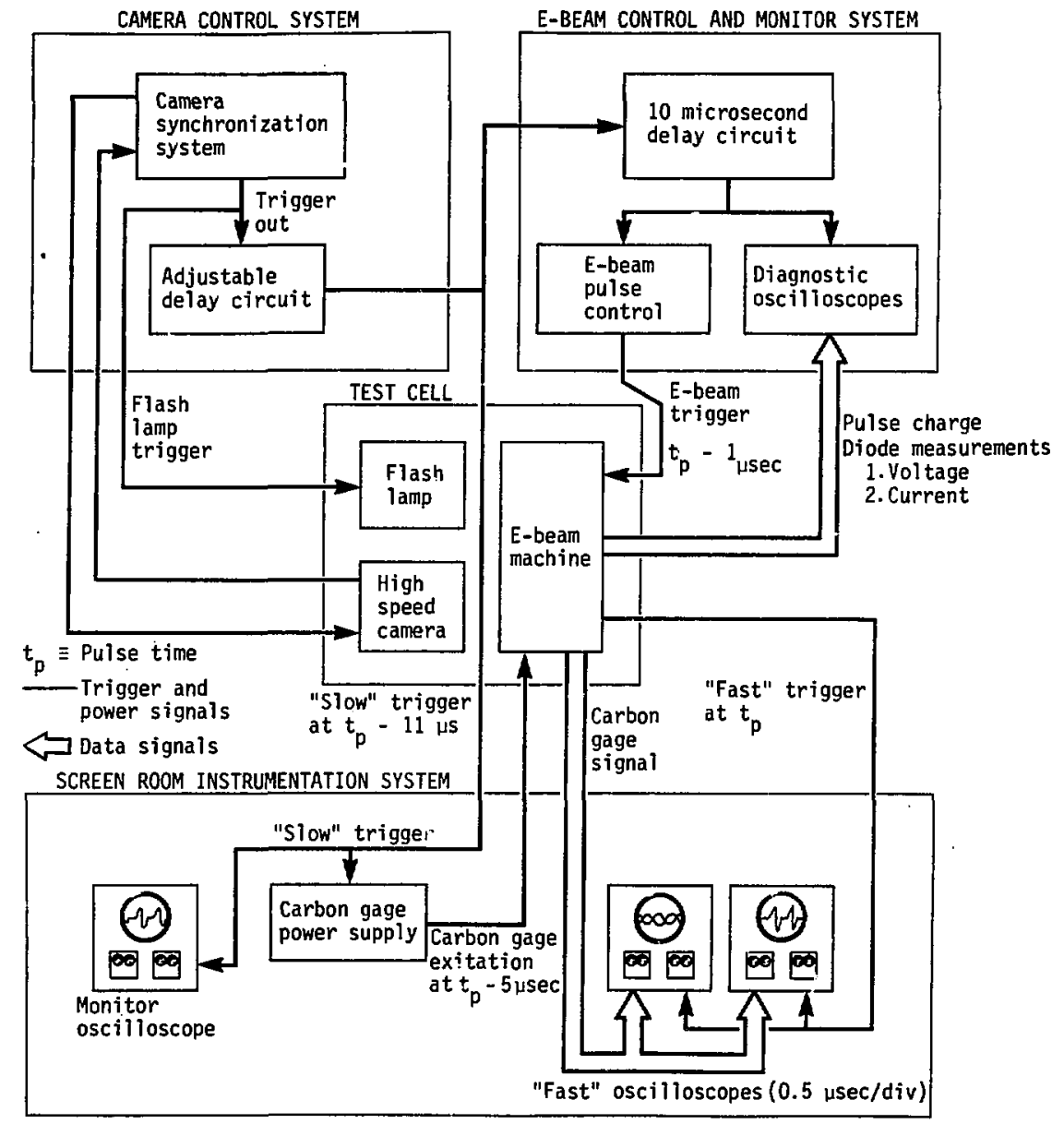

Figure 23. - The timing and firing circuitry is outlined here. The trigger from the camera control rack passes throurh the e-beam test cell to start the carbon gage power supply and the monitor oscilloscope. The fast trigger is produced by the e-beam discharge and triggers the fast oscilloscopes that record the carbon gage data. 


\section{LABORATORY PROCEDURE}

The experiments on the pulserad 422 were cr tucted continuously in blocks of time that were two to three weeks long. The machine was operated only one eight hour shift per day. A typical daily schedule is described in the following section.

Routine maintenance was done before inspecting and cleaning the diode chamber, the anode and cathode assemblies, and the target fixture apparatus. Following the cleaning and repair period, the e-beam was pulsed vith the nine-segment calorimeter installed in place of the lead target and tamper assembly. The machine was discharged in this configuration until it was evident that a stable set of pulse characteristics had been achieved. The procedure for discharging the beam follows:

1. The aluminun anode was installed over the opening in the wall of the diode chamber. The target assembly was placed over the anode foil and attached to the outside of the diode chamber (see Figure 7). The test (drift) chamber was then placed over the target assembly fixture and secured.

2. A mechanical vacuum pump then reduced the pressure in the two chambers to less than 0.1 torr in approximately 5 minutes.

3. The diode chamber was then switched to a diffusion vacuum pump and the pressure in the diode chamber was reduced to less than $1.0 \times 10^{-4}$ torr. Simultaneously a controlled amount of dry nitrogen was continuously supplied to the drift chamber until the desired pressure ( 2.0 torr for these tests) was achieved. This phase required five to 15 minutes, depending on the pumping power of the diffusion pump and the contamination level in the diode chamber.

4. After the desired pressures had been achieved, safety interlocks were set to prohibit the machine from discharging if anyone entered the test area after the firing sequence had been 
started. The firing sequence consisted of: 1) charging the capacitors in the Marx generator to $95 \mathrm{kV}$; 2) activating the diagnostic equipment; and 3$)$ triggering the Marx generator.

5. Following the discharge pulse, the various diagnostic and experiment data records were examined and entered in the shot log book. For simple experiments, (such as measuring only total fluence) the turn around time was approximately 45 minutes to one hour. Complicated data tests and the repair of occasionally damaged parts extended the required time so that only two data tests could be made each day.

The general procedure from day to day within the period of these experiments was to make several tests with the nine-segment calorimeter in place of the test fixture. These runs would establish the general operating level of the machine. The e-beam could vary as much as $30 \%$ in overall fluence output from day to day. This is a characteristic of the e-beam at maximum power output and attempts to improve it by rigorous control of all the test conditions available to the experimenter were not successful. This variation appears to be a problem of beam stability between the anode window and the target. At pulse energies less than one-half that required for these experiments, the beam is weil behaved and is repeatable to within $10 \%$.

Among the interesting but unexplained effects was a $50 \%$ reduction of the fluence when a brass guide cone replaced the copper cone. It was not thought that the electrical conductivity would make such a significant difference, and in $f a c t$ in other configurations the brass cones and pipes seem to work as well as the copper ones. 
When the machine and auxiliary test apparatus were considered to be operating stabily, test shots would be alternated with lluence measurements for the remainder of the day. This was typically two or three data tests. At selected times during the test series a day would be devoted to fluence transmission measurements. These measurements of the fraction of fluence that would penetrate various thjcknesses'of titanium provided the best estimate of the mean electron energy of the pulse and hence how much energy actually would be trapped in the lead targets. 


\section{EXPERIMENTAL RESULTS}

\section{TEST SAMPLE ASSEMBLY}

The conditions under which the final data were obtained are summarized in Table 2. As indicated, lead target thicknesses of 0.025

Table 2. Geometry of experimental test conditions.

\begin{tabular}{cccc} 
Case & $\begin{array}{c}\text { Lead thickness } \\
(\mathrm{mm})\end{array}$ & $\begin{array}{c}\text { Tamper thickness } \\
(\mathrm{mm})\end{array}$ & $\begin{array}{c}\text { Gaps } \\
(\mathrm{mm})\end{array}$ \\
\hline 1 & 0.025 & 0.125 & $0^{\mathrm{a}}$ \\
3 & 0.025 & 0.125 & 0.025 \\
4 & 0.025 & 0.125 & 0.50 \\
5 & 0.025 & 0.250 & $0^{\mathrm{a}}$ \\
6 & 0.025 & 0.250 & 0.025 \\
7 & 0.025 & 0.250 & 0.50 \\
8 & 0.050 & 0.125 & $0^{\mathrm{a}}$ \\
9 & 0.050 & 0.125 & 0.025 \\
11 & 0.050 & 0.125 & 0.50 \\
12 & 0.050 & 0.250 & $0^{\mathrm{a}}$ \\
\hline 10 & 0.050 & 0.250 & 0.05
\end{tabular}

a. - Lead bonded to tamper and buffer to insure void-free assembly.

$\mathrm{mm}$ and $0.05 \mathrm{~mm}$ and tamper thicknesses of $0.125 \mathrm{~mm}$ and $0.25 \mathrm{~mm}$ were used. The initial gaps between the lead target and the fused quartz buffer plate and the gaps between the lead and the titanium tamper were varied 
trom zero to $0.5 \mathrm{~mm}$. The smaller lead target and titanium tamper thicknesses were selected on the basis of the minimum sizes which could be handled and accurately installed in the test fixture. The larger sizes were based on permitting a significant fraction of the electron beam to penetrate and maintain a reasonably uniform energ; deposition profile through the lead.

In order to achieve a reliable zero gap condition, the tamper and fused quartz were bonded to the lead with a very thin layer of EPO-TEK 301 epoxy bonding material manufactured by Epoxy Technology, Inc. It was determined that when carried out in a vacuum environment, this low viscosity epoxy resulted in bond thicknesses less than $0.0015 \mathrm{~mm}$. The effect of this miniscule contaminent was investigated by making a zero gap test with no lead inslalled; the titanium lamper was bonded directly to the fused quartz. When the electron beam heated this configuration, negligible carbon gage stress and tamper motion were recorded.

\section{STRESS MEASUREMENTS}

The carbon element stress gages consistently yielded reliable data for the stress transmitted through the fused quartz buffer plate. The electron beam energy was low for case 4 (Table 2). This decreased the mass of lead vaporized by a factor of two and invalidated the data for that condition. Figure 24 shows a typical carbon gage data record and Table 3 lists the peak stresses indicated by the carbon gage. The recorded time of arrival of the various stress waves incident on the 


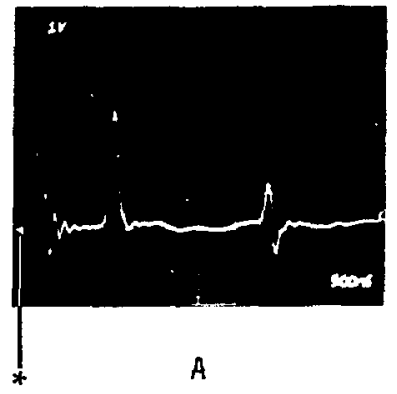

Vertical scale: 1 volt/div Horizontal scale: $0.5 \mu \mathrm{sec} / \mathrm{div}$

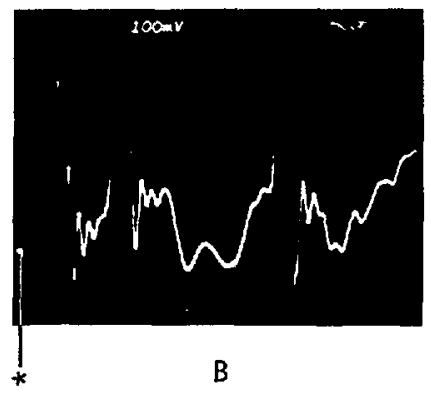

Vertical scale: $0.1 \mathrm{volt} / \mathrm{div}$ Horizontal scale: $0.5 \mu \mathrm{sec} / \mathrm{div}$

* E-beam pulse time

Figure 24. - Carbon gage data records for e-beam shot 7897. Trace B shows the detail of the stress wave between the two peaks. The second peak is the result of the wave reflected from the carbon ge ze interface returning from the lead-quartz interface. The trigger signal was generated by the diode current monitor with less than 5 nanoseconds jitter. 


\section{Table 3. Peak stress in carbon gage.}

\begin{tabular}{|c|c|c|c|c|}
\hline Case & $\begin{array}{l}\text { Lead } \\
\text { thickness } \\
\because \text { (mo) }\end{array}$ & $\begin{array}{c}\text { Tamper } \\
\text { th i ckness } \\
\text { (mn) }\end{array}$ & $\begin{array}{l}\text { Gaps } \\
\text { (mm) }\end{array}$ & $\begin{array}{c}\text { Peak stress } \\
(\mathbf{k b})\end{array}$ \\
\hline 1 & 0.025 & 0.125 & 0 & 9.1 \\
\hline 2 & 0.025 & 0.125 & 0.025 & 0.5 \\
\hline 3 & 0.025 & 0.125 & 0.50 & 0.2 \\
\hline $4^{\circ}$ & 0.025 & 0.250 & 0 & - \\
\hline 5 & 0.025 & 0.250 & 0.025 & 0.9 \\
\hline 6 & 0.025 & 0.250 & 0.50 & 0.2 \\
\hline 7 & 0.050 & 0.125 & 0 & 13.0 \\
\hline 8 & 0.050 & 0.125 & 0.025 & 4.5 \\
\hline 9 & 0.050 & 0.125 & 0.50 & 0.1 \\
\hline 10 & 0.050 & 0.250 & 0 & 13.0 \\
\hline 11 & $\quad 0.050$ & 0.250 & 0.025 & 5.0 \\
\hline 12 & 0.050 & 0.250 & 0.50 & 0.1 \\
\hline
\end{tabular}

a. No valid data obtained for this condition.

gage can be used to estimate the usable portion of the data record. The important points to note in tie signal shown in Figure 24 are:

1. - The sweep of the oscilloscope is triggered by the electron beam and the left edge is considered to be "zero" time.

2. - The initial ringing signal which decays in one to two microsecorids is the noise induced by the electron beam pulse:

3. - The tirst stress wave transtts the fused quartz buffer and produces a stress pulse at about $1.2 \mathrm{microsecands.}$ 
4. - A portion of the stress wave incident on the carbon gage is reflected back toward the lead-quartz interface where it is again reflected back toward the carbon gage.

5. - The reflected wave makes two transits of the fused quartz and arrives back at the carbon gage 2.4 microseconds after the initial wave passed.

6. - The combination of incident and reflected stress waves produce tension at the carbon gage at 3.7 microseconds.

The calculated transit time for the $6.35 \mathrm{~mm}$ buffer plate is 1.2 microseconds which is consistent with the arrival times observed on the data record. This indicates that the buffer plate remained intact and is capable of supporting the stress wave for this period of time. The carbon stress gage as noted in section 11, is not capable of taking tension loading. The calibration of the gage cannot be relied upon after it has been subjected to a tensile loading and thus the usable data portion of the record ends at 3.6 microseconds.

The data recorded from the stress gages are voltage changes at the bridge output terminal. The volts $\left(V_{t}\right)$ were converted to a fractional change in resistance $\left(\Delta R / R_{0}\right)$ using equation (9).

$$
\frac{\Delta R}{\mathrm{R}_{0}}=\frac{\mathrm{V}_{\mathrm{t}}\left[\begin{array}{c}
\mathrm{B} \\
\frac{-}{V_{\mathrm{g}}}
\end{array}\right]-\mathrm{A}}{1-\mathrm{V}_{\mathrm{t}}\left[\frac{\mathrm{C}}{\mathrm{V}_{\mathrm{g}}}\right]}
$$

Equation ( 9 ) is derived by solving the simultaneous equations that result from an analys of the circuit in Figure 20. The resistance, 
$R_{0}$. Is the sum of the carbon gage and caile resistances $\left(R_{g}+R_{c a}\right)$. For the nominal case where $R_{c a}$ is zero and $R_{g}$ is 50 ohms, the values for $A$, $B$, and $C$ are:
$A=0$.
$\mathrm{B}=3.15$
$\mathrm{C}=1.58$

Equation (9) gives the apparent $\Delta R / R$ whjch must be corrected to account for the cable resistance using Equation (10).

$$
\frac{\Delta R}{R_{g}}=\frac{\Delta R}{R_{0}}\left[1+\frac{R_{c a}}{R_{g}}\right]
$$

The cable resistance for these experiments was 1.38 ohms. The corrected value for the fractional resistance change was then converted to an apparent stress using the carbon gage calibration curve shown in Figure 19.

The noise signal coupled to the carbon gage at "zero" $t$ ime consistently caused the power supply to shut down the bridge exitation voltage within two microseconds which eliminated the passibility of getting carbon gage data. To solve this problem a passive network filter was added to the cable between the carbon gage and the bridge. The filter circuit is shown in Figure 25. The response of this filter to a voltage step is shown in Figure 26. Depending on the data signal pulse shape, this filter could cause attenuation of the data signal. A more thorough investigation of the filter response was therefore under taken. 


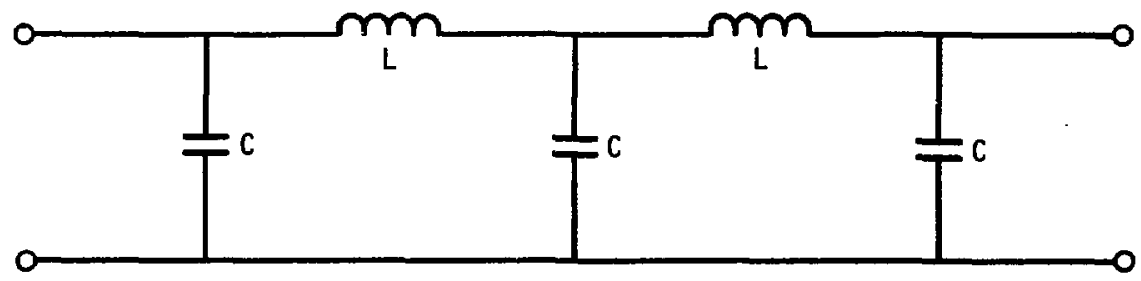

$$
\begin{aligned}
& L=1.8 \times 10^{-6} \text { henries } \\
& C=500 \times 10^{-12} \text { farads }
\end{aligned}
$$

Figure 25. - The filter used between the carbon gage power supply and the carbon gage element to reduce the possibility that the e-beam pulse would cause a malfunction in the power supply.

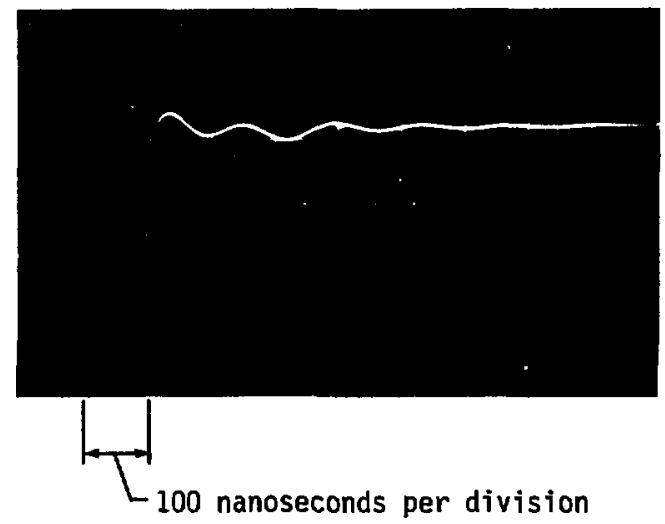

Figure 26. - The response of the carbon gage signal filter when subjected to a fast $r$ ising pulse. 
The carbon gage and tilter circuit elements shown in Figure 27a were used to construct a model for analyzing the effects of the filter on the response of the circuit to a changing gage resistance. The power flow bond graph'4 (Figure 27b) for the circuit aids in identifying the state equations necessary to describe the model response. The state 'equations in matrix form are:

$$
\underline{\dot{x}}(t)=\underline{A}(t) \underline{x}(t)
$$

which form a system of linear first order ordinary differential equations with $t$ ime varying coefficients. The expansion of $\underline{x}(t)$ is:

$$
X(t)=\left[\begin{array}{l}
q_{0}(t) \\
q_{1}(t) \\
\lambda_{1}(t) \\
q_{2}(t) \\
\lambda_{2}(t) \\
q_{3}(t)
\end{array}\right]
$$

with initial conditions,

$$
\underline{x}(0)=\left[\begin{array}{c}
q_{0}(0) \\
0 \\
0 \\
0 \\
0 \\
0
\end{array}\right]
$$

where $q$ is the charge developed on the capacitors and $\lambda$ is the $f$ lux linkage on the inductors. The expansion of the $\underline{\underline{A}(t)}$ matrix is given in Equation (14). 


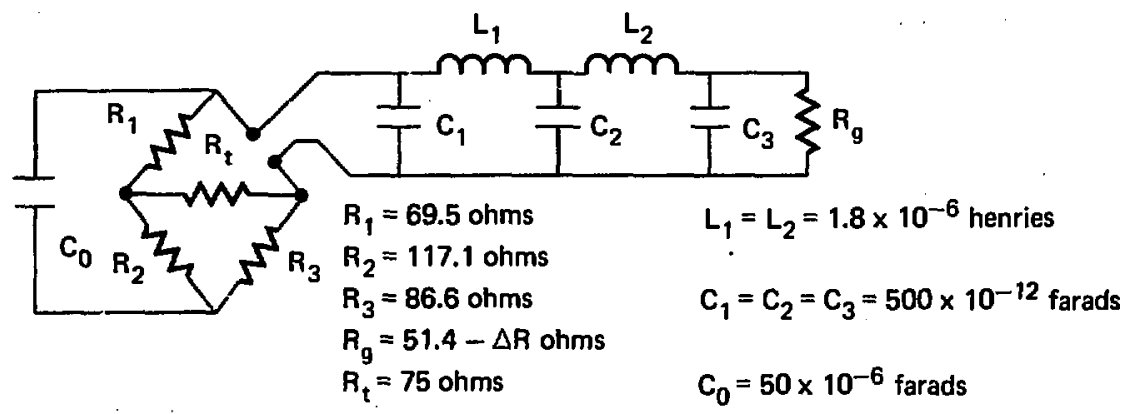

(a) Schematic diagram

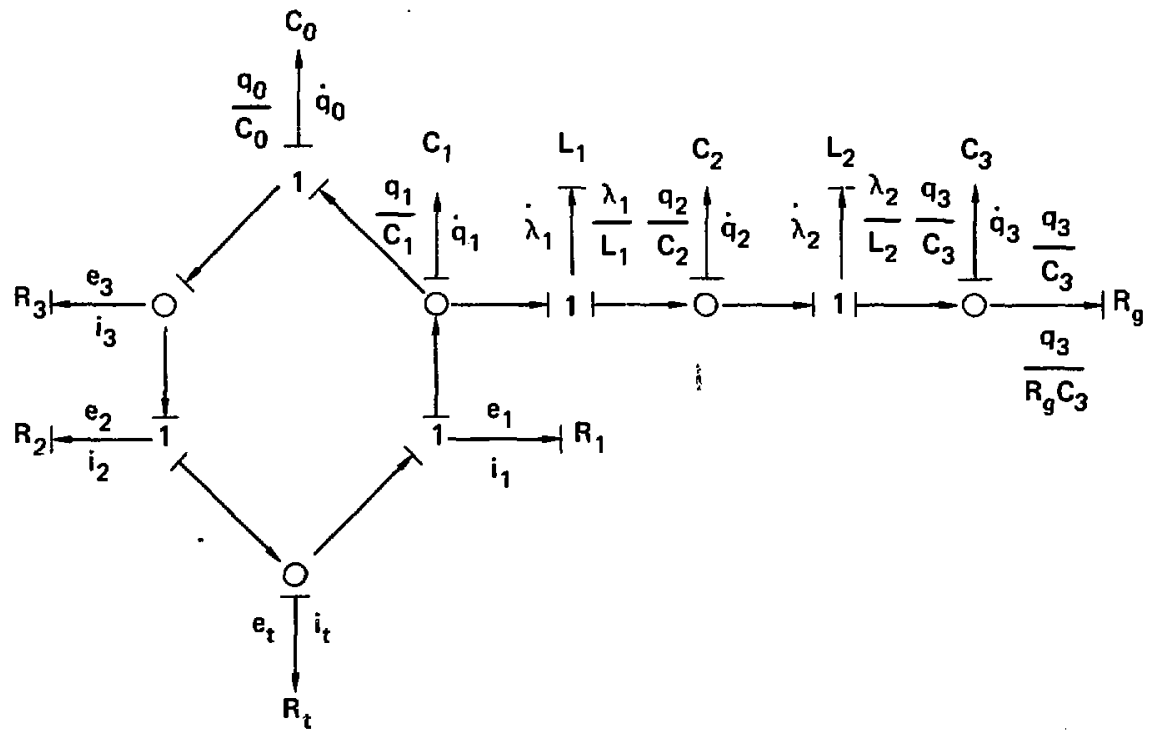

(b) Bond graph representation

Figure ?7. - Schematic diagram and bond graph representation of the carbon gage electrical circuit including the filter elements. 


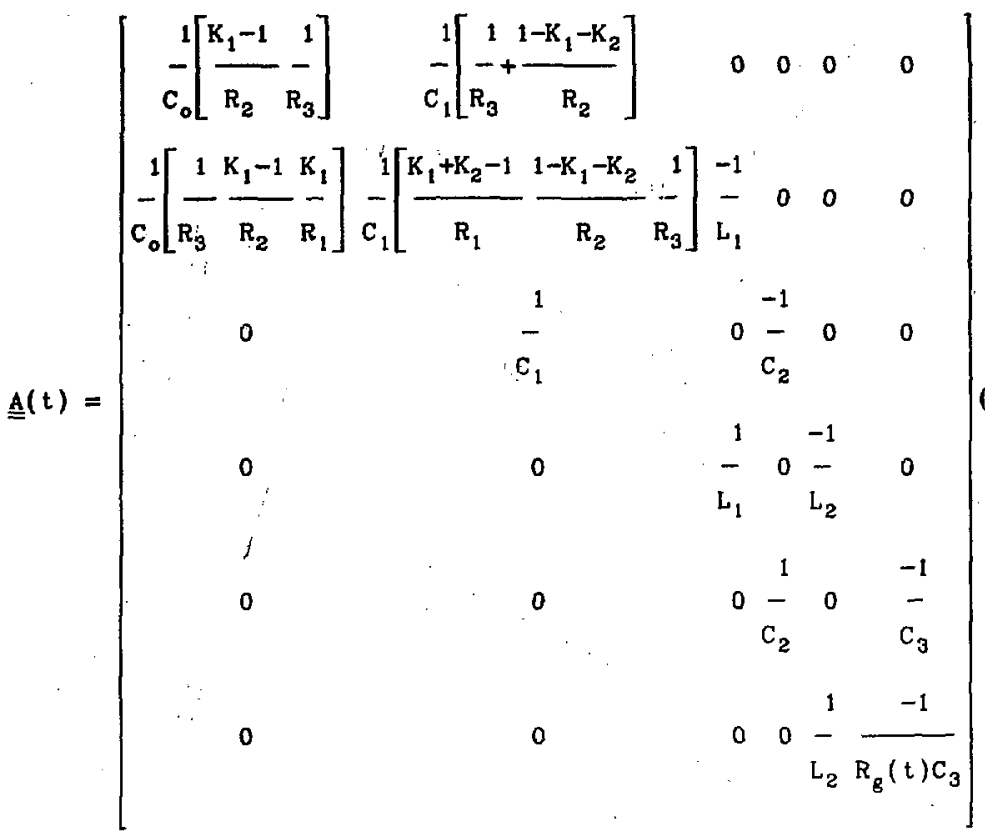

In Equation (14) $R_{g}(t)$ is $t$ ime varying and $K_{1}$ and $k_{2}$ are defined in Equations (15) and (16).

$$
\begin{gathered}
\mathrm{K}_{1}=\frac{\mathrm{R}_{\mathrm{L}} R_{1}}{\mathrm{R}_{\mathrm{t}} \mathrm{R}_{\mathrm{L}}+\mathrm{R}_{\mathrm{t}} \mathrm{R}_{2}+\mathrm{R}_{1} \mathrm{R}_{2}} \\
\mathrm{~K}_{2}=\frac{\mathrm{R}_{2}}{\mathrm{R}_{1}} \mathrm{~K}_{1}
\end{gathered}
$$

The signal, $v_{t}(t)$, measured by the oscilloscope as carbon gage output voltage is the voltage across $R_{L}$ (Figure $27 a$ ) and is given by Equation (17). 


$$
v_{t}(t)=k_{1} \frac{q_{0}}{c_{0}}+\left(k_{1}+k_{2}\right) \frac{q_{1}}{c_{1}}
$$

Time varying values of $R_{g}$ were computed in the hydrodynamic model of the experiment. The syslem of differential equations, equation (11), was integrated numerically using these variarions as input. Figure 28 shows the effect of the filter circuit on the predicted pulse. Although there is a time delay of about 50 nanoseconds in the filter, the peak response and the pulse width were changed less than six percent for all of the test conditions. The conclusion can be made that the effect of the filter can be nerlected wien comparing the peak response from the experiments with the corresponding predictions.

\section{VELOCITY MEASUREMENTS}

The high speed photographic records produced by the rotating mirror framing camera showed the titanium tamper position at two microsecnnd intervals. An example of some of the frames from one experiment is included as Figure, 29. The tamper and the lead mixture behind it are the opaque mass expanding toward the right side of the photographs. These profile views of the tamper were used to calculate the tamper velocity as a function of time. As can be noted the center of the tamper is accelerated to a greater velocity than the edges. This complicated the evaluation of the tamper velocity. 


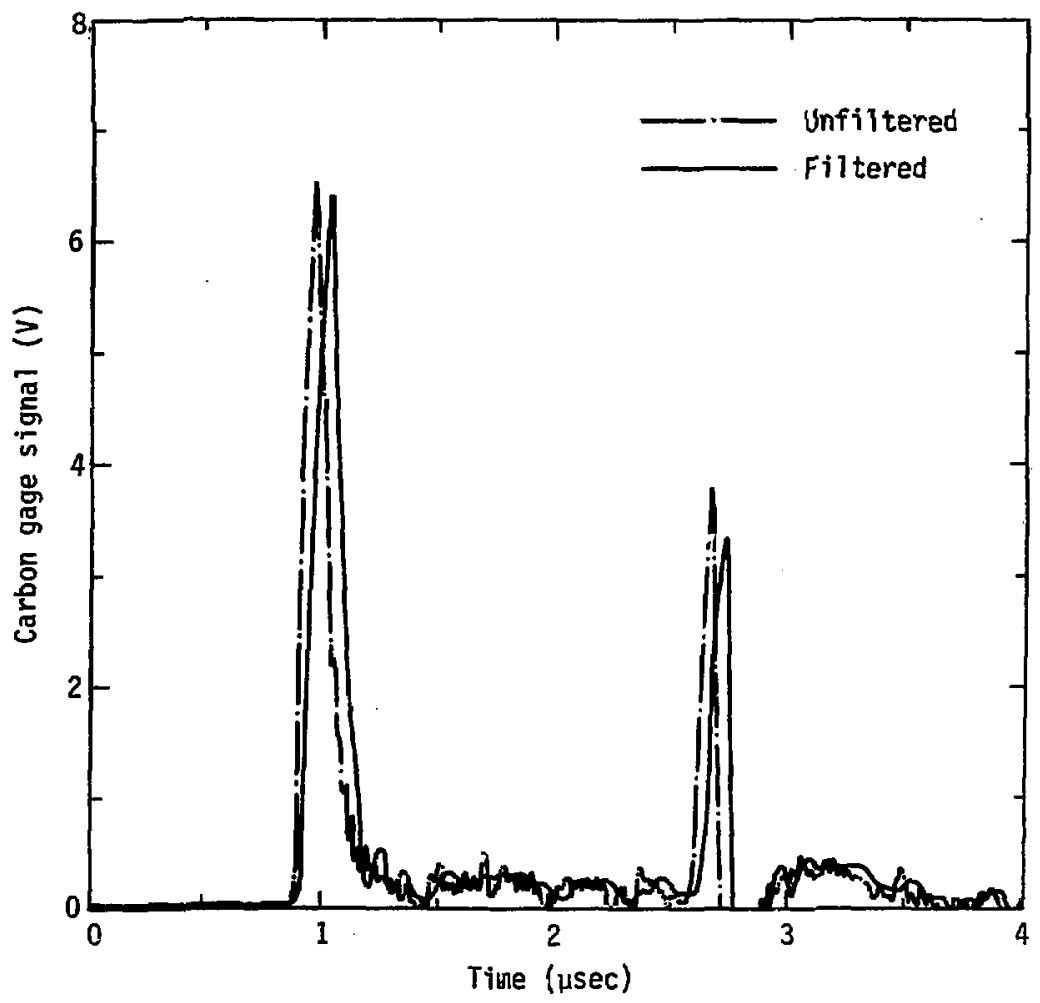

Figure 28. - Stress versus time histories illustrating the effect of the carbon gage filter circuit on the predicted stress calculation. 


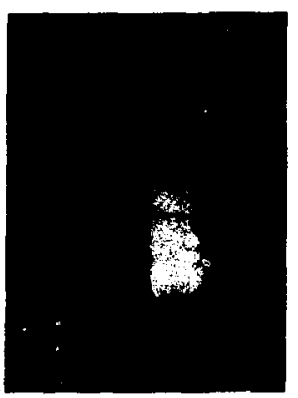

$0 \mu \mathrm{sec}$

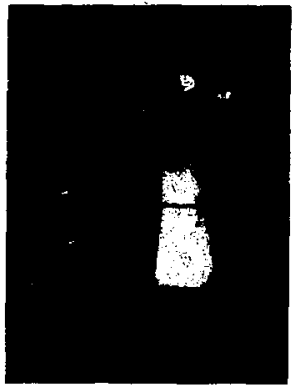

$10 \mu \mathrm{sec}$

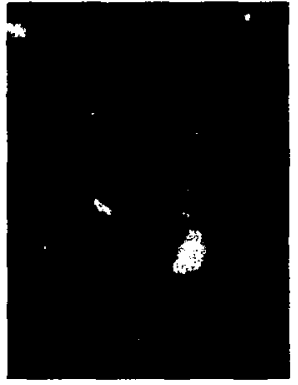

20 usec

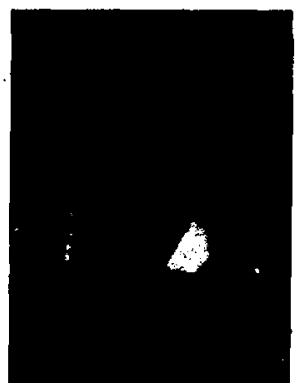

$30 \mu \mathrm{sec}$

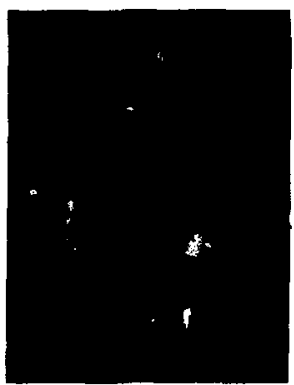

$40 \mu \mathrm{sec}$

\section{Experiment 7887}

Lead $-0.050 \mathrm{~mm}$; Tamper $-0.125 \mathrm{~mm} ;$ Gaps $-0.5 \mathrm{~mm}$

Figure 29. - A sequence of framing camera photographs of the tamper and lead motion. the horizontal line is part of the optical system in the camera. The dark area expanding to the right is the lead bounded by the tamper. The translucent area on the left is the quartz buffer ayer. 
Each frame of a record was magnified 15 times and projected on a rear surface viewscreen where the tamper contour was traced. The coordinates along this contour were recorded on a link from a time shared computer system. When each of the contours for a particular run had been entered, a program (VELOCITY) calculated the maximum and average tamper velocity as a function of position. The results of this calculation for the case in Figure 29 are shown in Figure 30 . The peaks and valleys are caused by errors in tracing the tamper profile. The edge of the tamper was not sharp which resulted in a random error of plus or minus four millimeters. This converts to a random velocity error of plus or minus $135 \mathrm{~m} / \mathrm{sec}$. These random errors were averaged out in $t$ ime to get the values in Table 4.

The column of maximum velocities in Table 4 is the velocity of the lastest moving portion of the tamper. The average velocity is the velocity of all sections of the tamper surface, averaged as if it were curved equally in the $\mathrm{Z}$ direction and $\mathrm{Y}$ direction. The maximum number of frames available was 25 to 28 depending on the camera synchronization jitter. The velocity of the tamper was high enough in some cases to cause it to move of the field of view as early as 16 microseconds. While in some cases, the tamper did not appear to reach an asymptotic velocity before the framing camera record ended, the bulk of the experimental data indicated that the measured value was within $20 \%$ of a steady velocity. 


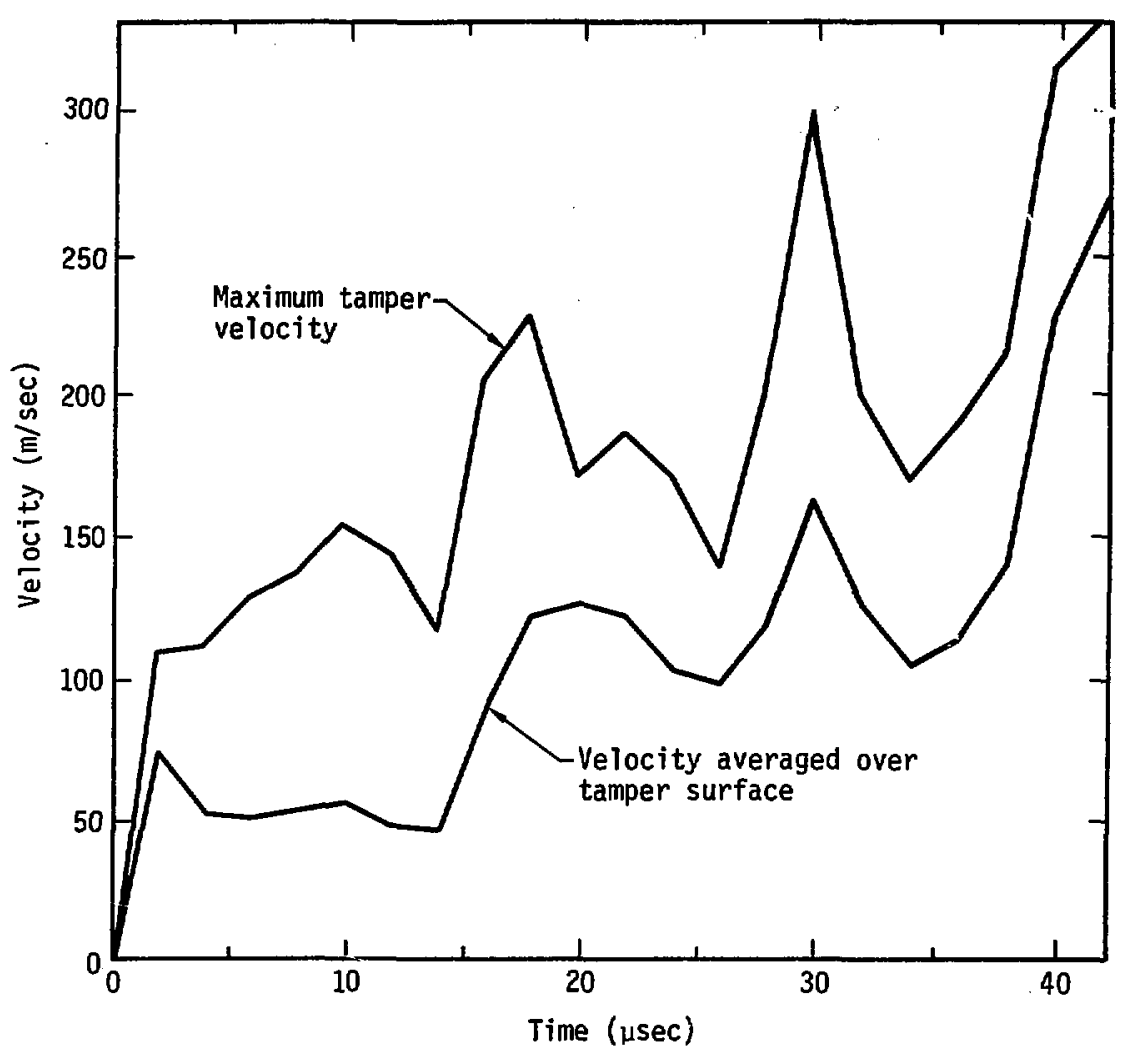

Figure 30. - The velocity of the tamper resulting from analysis of the framing camera photographs (Case 9 \& Figure 29) of tamper position at two microsecond intervals. 
Table 4. - Measured tamper velocity.

\begin{tabular}{|c|c|c|c|c|c|c|}
\hline Case & $\begin{array}{c}\text { Lead } \\
\text { thickness } \\
(\mathrm{mm})\end{array}$ & $\begin{array}{c}\text { Tamper } \\
\text { thickness } \\
\text { (mm) }\end{array}$ & $\begin{array}{l}\text { Gaps } \\
(\mathrm{mm})\end{array}$ & $\begin{array}{l}\frac{\text { Tamper }}{\text { Max. }} \\
\text { (m/sec) }\end{array}$ & 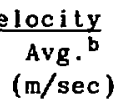 & $\begin{array}{l}\text { Time of } \\
\text { measurement } \\
\text { ( } \mu \text { sec) }\end{array}$ \\
\hline 1. & 0.025 & 0.125 & 0 & 300 & 300 & 16 \\
\hline 2 & 0.025 & 0.125 & 0.025 & 170 & 120 & 50 \\
\hline 3 & 0.025 & 0.125 & 0.50 & 100 & 80 & 28 \\
\hline $4^{c}$ & 0.025 & 0.250 & 0 & - & - & - \\
\hline 5 & 0.025 & 0.250 & 0.025 & 80 & 50 & $5 u$ \\
\hline 6 & 0.025 & 0.250 & 0.50 & 50 & 30 & 35 \\
\hline 7 & 0.050 & 0.125 & 0 & 500 & 400 & 22 \\
\hline 8 & 0.050 & 0.125 & 0.025 & 260 & 140 & 40 \\
\hline 9 & 0.050 & 0.125 & 0.50 & 250 & 200 & 40 \\
\hline 10 & 0.050 & 0.250 & 0 & 250 & 220 & 30 \\
\hline 11 & 0.050 & 0.250 & 0.025 & 160 & 120 & 35 \\
\hline 12 & 0.050 & 0.250 & 0.50 & 80 & 50 & 35 \\
\hline
\end{tabular}

An attempt to reduce the random errors associated with the $15 x$ projection and tracing of the tamper profiles was made. Each frame on the films from several experiment runs was scanned with a digitizing microdensitometer. In this process the optical density of the film was recorded through a 20 micrometer aperture at 20 micrometer intervals. These data provided the input to a computer program which plotted constant density contours for each frame. These contours were then used 
to determine the location of the tamper edge in each frame. This procedure did not reduce the frame to frame random variations significantly; but did yield results in agreement with (and thus increased the confidence in) the previously calculated average values for tamper velocity.

The bending of the tamper is caused by non-uniform distribution of f Juence on the target. A dynamic analysis of the lamper plate using an initial velocity distribution showed the same order of curvature as the experimental data. These results are shown in Figure 31 . The calculations were made using a finite element mesh computer program DTVIS2 $2^{15}$ with the initial velocities corresponding to velocities predicted for the typical fluence pattern measured with the nine-segment calorimeter inside the test fixture. The initial velocity of the interior tamper zones was taken relative to the outer edge which was arbitrarily assigned a zero initial velocity. This kept the iamper from moving too far from the reference grid.

\section{LOW PRESSURE MEASUREMENTS}

An attempt was made to use a fast response diaphragm type pressure gage to measure the lead vapor pressure in the expanding cavity. The type of transducer that appeared to be applicable was a miniature (two mm diameter) gage with a silicon diaphragm. A semiconductor strain gage that'measured diaphragm deflection was an integral part of the silicon 


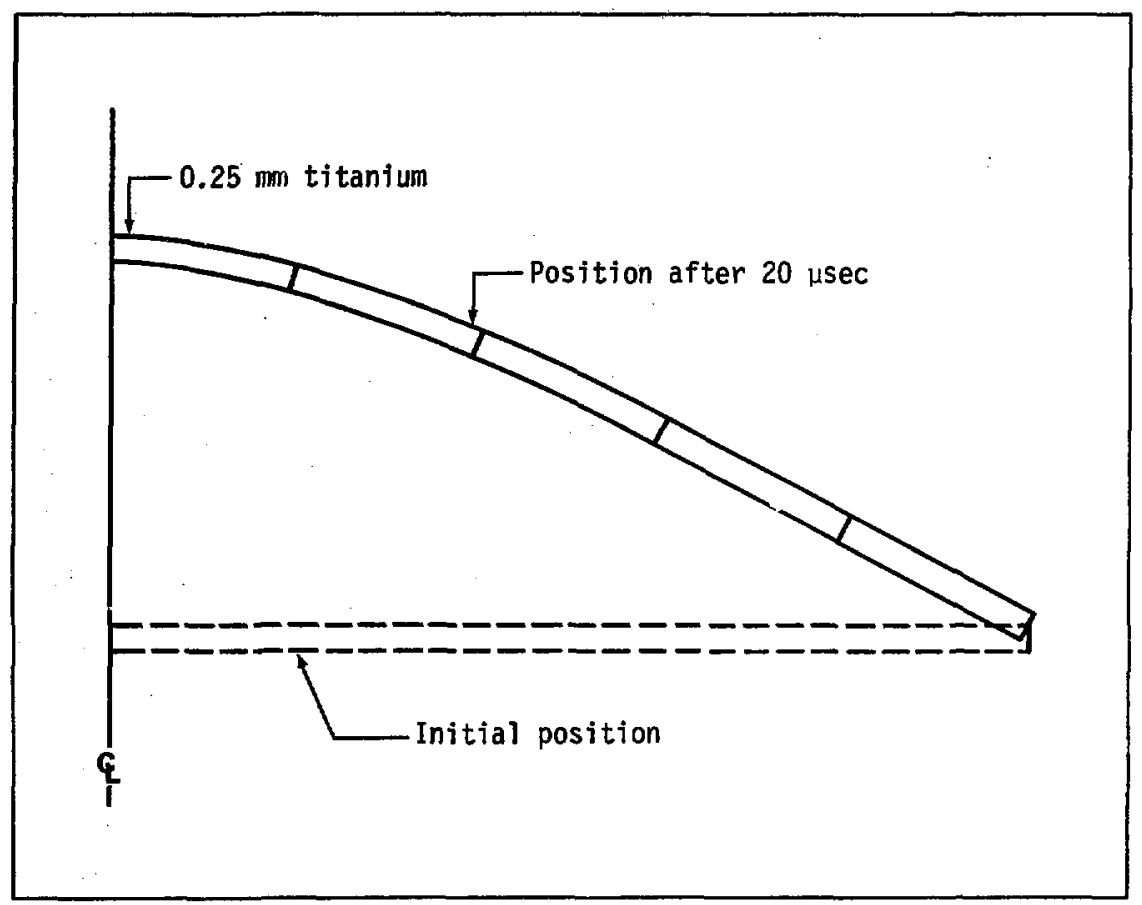

Figure 31. - The tamper shape predicted for a non-uniform initial velocity distribution. A dynamic finite element structural analysis was used. 
element. This transducer was protected from the initial expansion of the lead mixture by a silicon rubber pad.

This attempt was unsuccessful. The impact of the liquid-vapor lead apparently caused the gage to fail before low pressure data could be recorded. The transducer was mounted in the top of the $f$ ixture and survived repeated pulsing of the electron beam when no lead target was installed. This is an indication that the failure was mechanical or thermally induced rather than due to excessive electrical currents. Small silicon rubber pads were used to cushion the impact, but even though the pads were not penetrated or damaged, the pressure gage was inoperative following the test. 


\section{PREDICTED RESULTS}

\section{HYDRODYNAMIC MODEL}

Predicting the behavior of a dynamic process requires a model that will compute the distributions of pressure, density, velocity, and energy in the material as tunctions of time. The equations for calculating each of these can be derived from consideration of the laws of conservalion of momentum, mass, and evergy plus a constitutive equation of state (EOS) relating pressure to energy and density. The computer program that was used to perform these calculations was the Lawrence Livermore Laboratory's one-dimensional ${ }^{\dagger}$ hydrodynamics program, KOVSPALL, a version of $\mathrm{KO}^{18}$ that includes the GRAY ${ }^{17}$ three phase analytical EOS. KO and KOVSPALL utilize a Lagrangian coordinate system wherein the coordinates move with the mass elements of the material being studied. Each mass element has the following properties associated with it:

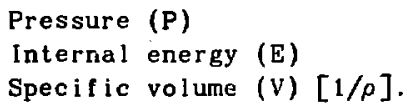

Most solid materials have properties that resist change in shape. If

t - There are two and three dimensional hydrodynamic computer programs available. The multi-dimensional analysis methods use the same techniques to model system behavior, but the equations are modified to calculate ross-axis stresses and motions. The KO-HEMP series uses GRAY and other EOS models developed at LLL, and the CHART-CSQ tamily developed by. Thompson at Sandia Laboratories in Albuquerque have the ir own EOS program ${ }^{18}$. Utilization of a 2-D or 3-D program was not practical because of the greatly increased computing time requirements for comparable zoning detail. 
the restoring forces vary linearly with distortion, the material behaves elastically. The combination of the pressure due to pure volume change, commonly called the hydrostatic pressure, and the distortion component, commonly called the stress deviator, create a net force or stress on the zone boundary ${ }^{18}$.

Each zone has two boundaries which are moving. The net stress on the boundary of adjacent zones and the zone masses are used to calculate the acceleration of the boundary. The acceleration of both boundaries of the zone can be integrated to obtain the change in volume. The new relative volume, plus any addition or subraction of energy to the zone can be used to determine the new pressure. The pressure is calculated from the EOS of the material. The equations of motion are integrated numerically in a consistent and energy conserving manner in the hydrodynamics program.

The self consistent units used in KOVSPALL are length in centimeters, time in microseconds, pressure in megabars (one megabar = $10^{12}$ dynes $\left(\mathrm{cm}^{2}\right)$, and energy in megabar- $\mathrm{cm}^{3}$. Figure 32 is a schematic diagram of the way the materials in the experiment were divided up for the KovsPall calculation. Table 5 lists the details of the geometric parame ters.

For the $f$ irst 50 nanoseconds energy was added to the lead and fused quartz regions. Energy addition to the titanium layer did not change the tamper response as long as the tamper remained solid. The rate of energy addition was constant at each zone, but varied with depth 
Titanium tamper:

20 evenly spaced zones

Fused quartz:

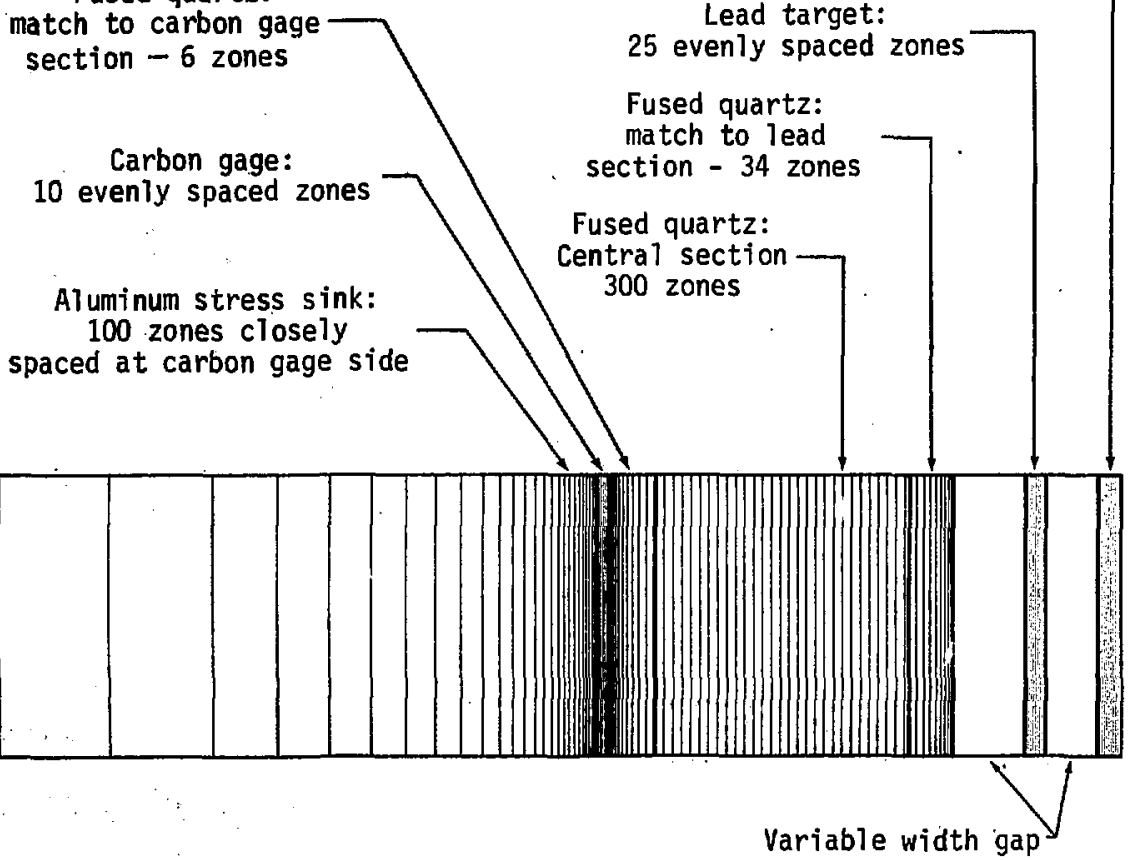

Variable width gap

Figure 32. - Zoning arrangement used for the hydrodynamic computer program simulation of the e-beam experiment. Zoning in the lead, tamper, and carbon gage areas was made quite detailed. The zones in the fused quartz were made thin enough to eliminate artificial attenuation of the stress wave. The total number of zones used was 495 out of a maximumavallable 500 . 
Table 5. - Geometric properties of the analytical model.

\begin{tabular}{|c|c|c|c|c|c|c|}
\hline \multirow[b]{2}{*}{ Region } & \multirow[b]{2}{*}{ Material } & \multicolumn{3}{|c|}{ Zone thickness } & Number & \multirow{2}{*}{$\begin{array}{l}\text { Region } \\
\text { width } \\
(\mathrm{cm})\end{array}$} \\
\hline & & $\begin{array}{l}\text { Lower bound } \\
\qquad(\mathrm{cm})\end{array}$ & $\begin{array}{l}\text { Upper bound } \\
(\mathrm{cm})\end{array}$ & $\operatorname{Rat}$ io ${ }^{a}$ & of zones & \\
\hline 1 & aluminumb & 0.045300 & 0.005657 & 1.0365 & 51 & 1.1250 \\
\hline 2 & aluminum & 0.005457 & 0.001300 & 1.0365 & 49 & 0.1250 \\
\hline 3 & carbon gage & 0.006500 & 0.006500 & 1.0 & 10 & 0.0065 \\
\hline 4 & fused quartzc & 0.001500 & 0.001992 & 1.0583 & 6 & 0.0110 \\
\hline 5 & fused quartz & 0.001947 & 0.001947 & 1.0 & 300 & 0.5840 \\
\hline 6 & fused quartz & 0.001948 & .0 .003000 & 1.0583 & 34 & 0.0300 \\
\hline 7 & gap & $\therefore-$ & - & - & 0 & variable \\
\hline 8 & lead & 0.000100 & 0.000100 & 1.0 & 25 & 0.0025 \\
\hline 8. & lead & 0.000200 & 0.000200 & 1.0 & 25 & 0.0050 \\
\hline 9 & gap & - & $\cdot-$ & - & 0 & variable \\
\hline 10 & $t i \tan i u m$ & 0.000625 & 0.000625 & 1.0 & 20 & 0.0125 \\
\hline $10^{\circ}$ & titanium & 0.001250 & 0.001250 & 1.0 & 20 & 0.0250 \\
\hline
\end{tabular}

Notes:

a. Ratio of thicknesses of adjacent zones.

b. Aluminum is divided into two parts for edit purposes only. It could have been one 100 zone region.

c. Fused quartz is divided into three regions so that the thickness to stress wave velocity ratio could be preserved reasonably well across the interfaces with the lead and carbon gage without exceeding the 500 zones available for the problem.

according to the profile shown in Figure 10. The total amount of energy deposited in the 50 nanosecond period was 50 calories $/ \mathrm{cm}^{2} \therefore$ The energy added to the quartz caused only slight (less than 0.1 kilobar) pressure precursor waves. 


\section{EOUATION OF STATE}

The finite difference methods used by KOVSPALL to calculate the behavior of the materials require that the pressure associated with any mass element be obtainable from a knowledge of the density and energy of the element. This required functional relationship is very complex especially when phase changes are involved. The usual approach is to use equations applicable to specific phases only and to develop procedures for matching predictions at the boundaries between the phases. Considering the solid state first it is noted tinat this will be the only phase involved for the titanium, fused quartz, carbon gage, and aluminum. The Grünelsen 20 EOS, used here to describe the solid phase ,response, calculates the pressure in the compressed solid from the following equation:

$$
P(V, E)=P_{H}(V)\left[1-\frac{\mu \gamma(V)}{2}+\frac{\gamma(V)\left[E-E_{0}\right]}{V}\right]
$$

where:

$$
\begin{gathered}
P_{H}(V)=\frac{p_{0} C^{2}}{(1-S x)^{2}} \\
\mu=\frac{V_{0}-V}{V}
\end{gathered}
$$




$$
\mathrm{x}=\frac{\mu}{\mu+1}
$$

The term $\mathrm{P}_{h}(V)$ is the cold compression or "Hugoniot" pressure associated with a sudden adiabatic decrease in volume. The coefficients $C$ and $S$ are defined as

$$
u_{B}=c+s \cdot u_{p}
$$

where $U_{s}$ is the shock velocity and $U_{p}$ is the particle velocity. Equation (22). has been found to be an acrep'able representation of the $U_{s} \cdot U_{p}$ data measured in plane shock wave experiments for the sub-megabar pressures of interest here. The Grüeisen coefficient, $\gamma(V)$, is usually considered to be constant for the small changes in volume occurring during the compression processes. Table 6 lists the properties used for the materials which are found only as solids during the analytical modeling studies.

Modified versions of the Grüneisen EOS are usually used for the liquid phase and a form of gas law equation is used for the vapor state. GRAY is the three phase equation of state used in KOVSPALL to characterize material in all t'.ree phases. GRAY is based on the joining of an analytical scaling leir EOS developed by Grover's with a modified van der Waals EOS developed by. Young and Alder22. The joining of the two models takes place at a volume, $v_{j}$, equal to 1.24 times $v_{0}$. In this region the isotherms and isentropes for the two models have sufficiently simjlar ordinates and slopes to achieve a satistactory join. 
Table 6. EOS Properties for solid phase materials.

\begin{tabular}{|c|c|c|c|c|c|c|c|}
\hline Material & $\begin{array}{c}\text { Reference } \\
\text { density } \\
\left(\mathrm{gm} / \mathrm{cm}^{3}\right)\end{array}$ & $\begin{array}{l}\text { Yield } \\
\text { stress } \\
\text { (Mbar) }\end{array}$ & $\begin{array}{l}\text { Shear } \\
\text { stress } \\
\text { (Mbar) }\end{array}$ & $\frac{\mathrm{C}}{(\mathrm{cm} / \mu \mathrm{sec})}$ & $\mathbf{S}$ & G & Source \\
\hline
\end{tabular}

\begin{tabular}{lcllllll}
\hline Titaniuma & 4.51 & 0. & 0. & $0.47 \mathrm{i}$ & 1.015 & 1.11 & Steinberg $^{23}$ \\
Fused quartz & 2.20 & 0.0115 & 0.338 & 0.576 & -2.14 & 0.035 & van Tiel1 \\
Carbon gageb & 1.44 & 0. & 0. & 0.243 & 1.73 & 1.13 & Steinberg \\
Aluminum & 2.70 & 0.003 & 0.292 & 0.537 & 1.34 & 2.0 & Bakken $^{25}$
\end{tabular}

Notes:

a. Titanium is treated hydrodynamicly because of its high temperature (near melt).

b. Carbon gage is simulated by a polyimide/epoxy lamination and empirical data are used.

Temperature instead of energy as an independent variable can be used for the EOS surface. The two properties are related by the specific heat capacity of the material. The lemperature axis is more appropriate for the two phase region because the two phase pressure depends only on the temperature. The P,V,T surface will be much more complicated when it includes more than the compressed solid state. The surface for lead is shown in Figure 33.

Grover's scaling law EOS utilizes the Grüneisen EOS for the compressed solid region. In the solid to liquid transition and in the liquid phase, he makes use of the Lindemann Law which relates the melt temperature to specific volume and Richard's Law concerning the constancy of the entropy of melting. Grover points out that for most metals the temperature. dependence of the specif ic heat in the liquid is a universal. curve scaled with the melting temperature. These 


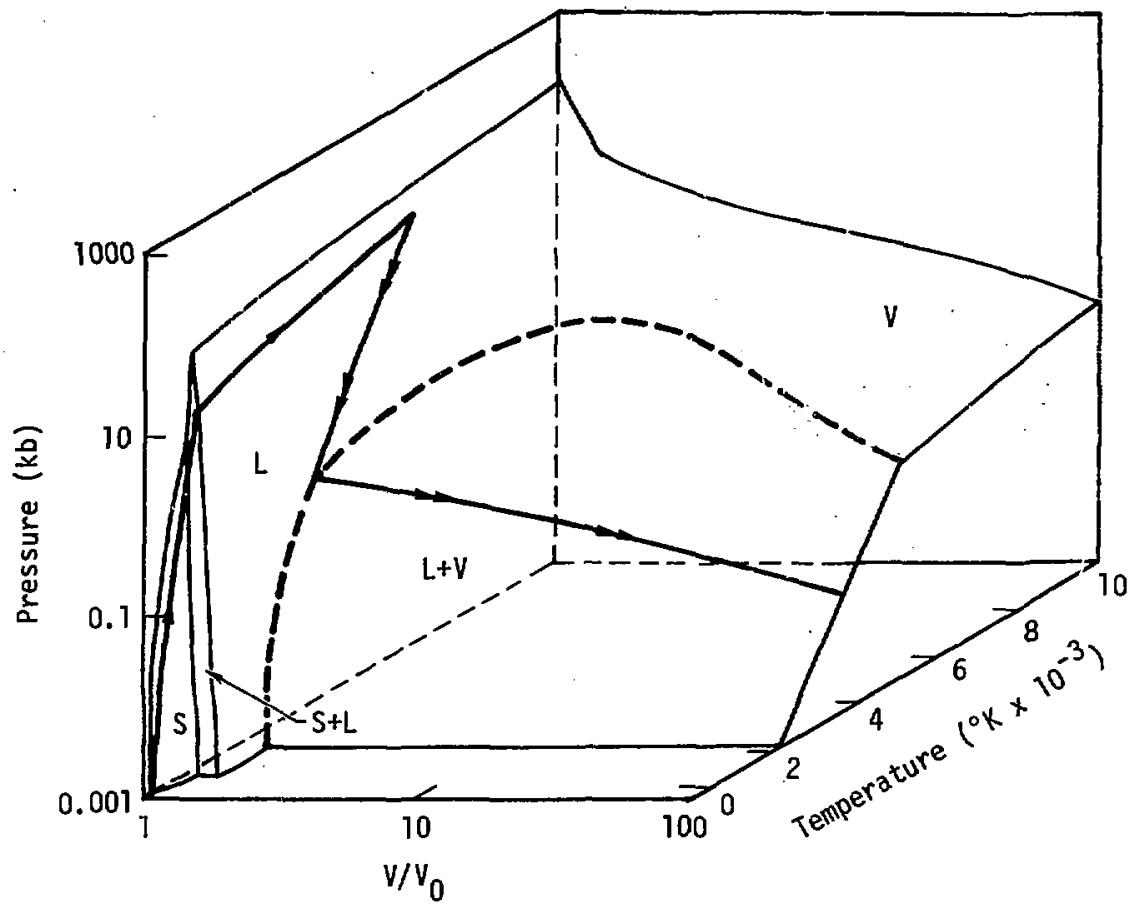

$\longrightarrow$ - Nearly constant volume to $5500^{\circ} \mathrm{K}$ Isentropic expansion -- - - Saturated liquid/vapor line

Figure 33a. - The GRAY equation of state surface for lead. 

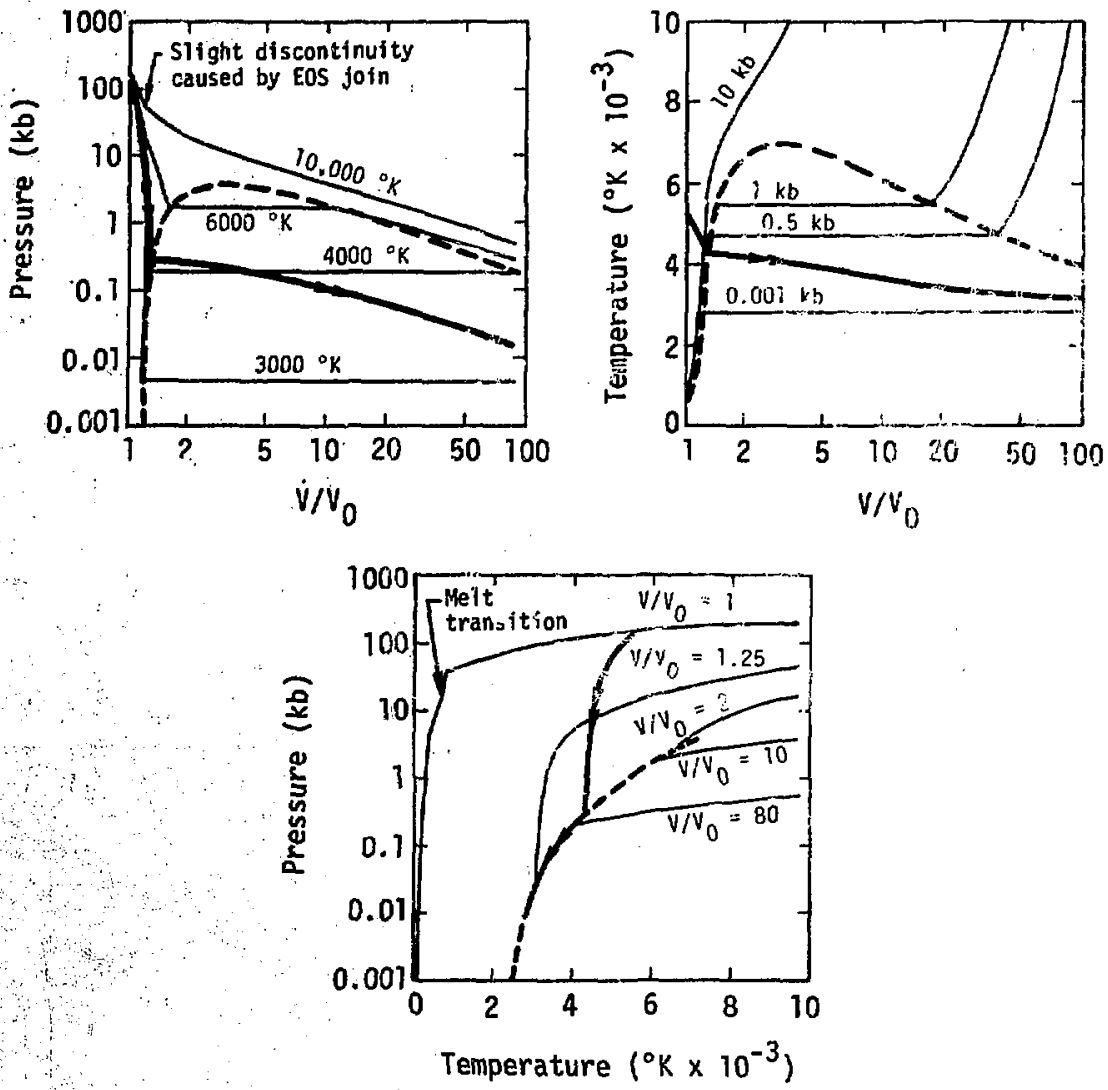

Isentropic expansion

$--\ldots-$ - Saturated liquid/vapor line

Figure 33b. - Projections of the GRAY EOS surface in the $P, V ; T, V$; and P T planes. 
relationshipg are used in arriving at an anatical description of the PVT surface at the transition plane and in the liquid adjacent to it.

The liquid to vapor transition and the extended vapor region is treated with the classic van der Waals EOS as modified by Young and Alder. This model improves the hard sphere repulsion term in the van der Waals equation and provides a means for estimating the coefficients associated with both the repulsion term and the van der Waals attraction term without resorting to critical point data.

Van der Waals equation gives rise to non-physical maxima and minima lor pressures along an isotherm in the liquid-vapor region. A method (originally suggested by van der Waals) for removing these unwanted "loops" establishes constant pressure tie lines which connect the high density liquid with the low density vapor. Using the Maxwell construction, a tie line pressure is delermined for a particular temperature such that the $\mathrm{p}-\mathrm{V}$ area above the line is quai to that below 26 . These corrections are part of the EOS as it is implemented in KOVSPALL. Table 7 ists the GRAY input properties supplied to KOVSPALL

\section{PREDICTED VARIATION OF PEAK QUARTZ STRESS AND TAMPER VELOCITY}

Figure 34 shows a representative predicted stress pulse as it is being propagated across the quartz buffer plate. The oscillations behind the peak pressure pulse are caused by reflecting stress waves in the liquid-vapor lead coupling into the fused quartz. It is not clear that this is a physical phenomenon or a numerical one, but regardless of 


\section{Atomic weight}

Reference specifle volume

Hugoniot reference energy

$U$, versus $U_{p}$ Hugoniot coefticient

$U_{\text {, versus }} U_{p}$ Hugoniot coefticient

Grüneisen coefl, of solid at $v_{0}$

Coelficient in volume dep. of $\gamma$

Melling Grüneisen coeff at $v_{0}$

Coefficient in volume dep. of $\gamma_{m}$

Electronic gamma

Electronic energy coefficient

Melt temperature at $v_{0}$

Constant entropy of melting

Excluded volume ratio

Attraction coefficient for vapor

Join volume ratio

Join parameter
Aw $\quad 207.2$

v $\quad 0.0882 \quad \mathrm{~cm}^{3} / \mathrm{gram}$

$\mathrm{E}_{\text {oH }} \quad 0$.

C $\quad 0.201 \mathrm{~cm} / \mathrm{sec}$

$\mathrm{S} \quad 1.54$

$\gamma_{0} \quad 2.84$

a $\quad 2.3$

$\gamma_{\text {om }} \quad 2.84$

$a_{m} \quad 2.3$

$\gamma_{e} \quad 2 / 3$

$\mathrm{g}_{\mathrm{e}} \quad 14.7 \times 10^{-9} \quad \frac{\mathrm{Mbar} / \mathrm{cm}^{3}}{\mathrm{~mole}-{ }^{\mathrm{a}} \mathrm{K}^{2}}$

$\mathrm{T}_{\mathrm{mo}} \quad 760 \quad{ }^{\circ} \mathrm{K}$

$\Delta S \quad 9.637 \times 10^{-5} \quad \frac{\mathrm{Mbar}-\mathrm{cm}^{3}}{\mathrm{moi}=-{ }^{\circ} \mathrm{K}}$

$\mathrm{v}_{\mathrm{b}} / v_{\mathrm{o}} \quad 0.48$

$a_{Y} \quad 49 . \quad \frac{\text { Mbar }-\mathrm{cm}^{8}}{\text { mole }^{2}}$

$v_{j} / v_{0} \quad 1.24$

$\theta \quad 1.0$ 


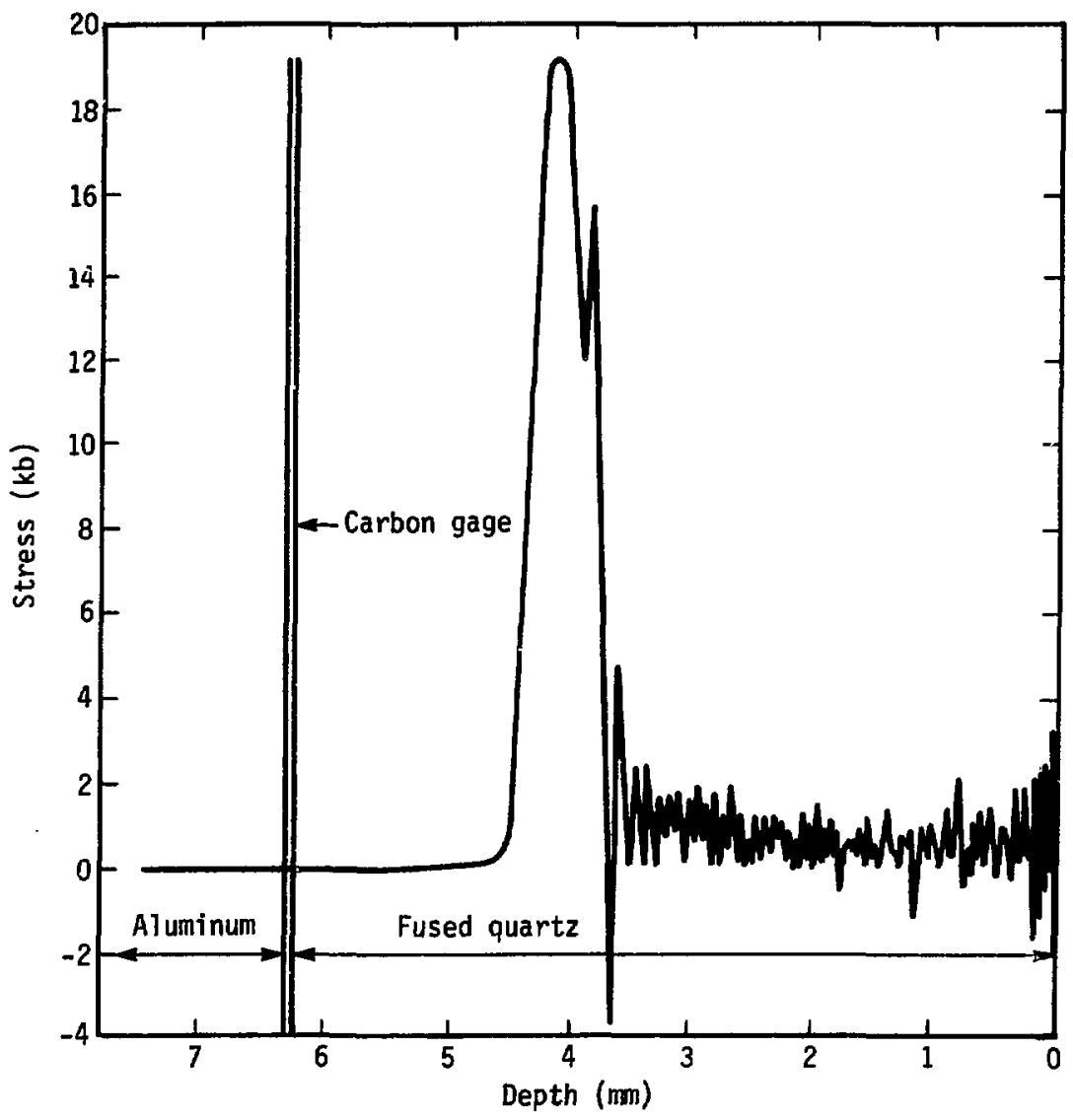

Figure 34. - The stress pattern predicted by KOVSPALL for the thick $(0.05 \mathrm{~mm})$ lead and thick $(0.25 \mathrm{~mm})$ tamper initially bonded in place (i.e. no gaps). The time at which this "snapshot" was plotted was 0.6 microseconds after energy deposition. 
origin, it is attenuated significantly by the relatively slow rise time of the carbon gage installation. This effect can be noted by comparing Figure 35. the time history of the stresy from the carbon gage for the same case, with Figure 34.

The value of the peak stress occurring at the carbon gage location was selected for comparison with the experimental measurements. Predicted variations ${ }^{\prime}$ with initial gap size are plotted in Figures 38 and 37. The experimentally determined results for three gap sizes are also plotted.

Figure 38 is the predicted tamper velocity versus time for the $0.025 \mathrm{~mm}$ lead and $0.125 \mathrm{~mm}$ tamper with no gaps. It has a very fast initial rise to more than $50 \%$ of its asymptotic value in the first microsecond. Figures 39 and 40 show the predicted tamper velocity at 50 microseconds as a function of the initial gap size. There is not nearly as much attenuation of the tamper velocity with gap size as the experimental data would suggest. This will be a topic for discussion in the next section.

1 - Computer runs werc typically made for process times of 6 to 50 microseconds. This required machine (CDC 7600) times of up to 60 minutes por run. These times reflect the fact that the program used has a very general hydrodynamic model. 


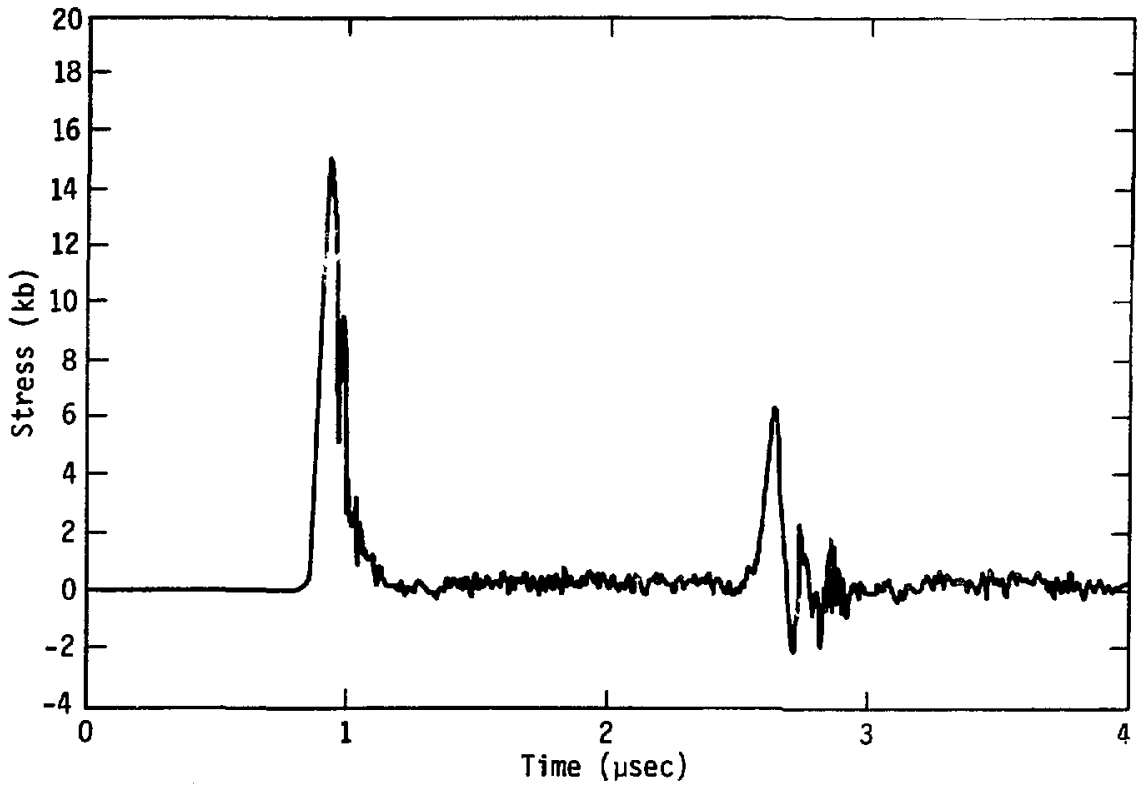

Figure 35. - Predictions of the stress developed in the carbon gage. The stress plotted is calculated at the center of the carbon gage installation. The carbon gage filter circuit delays the signal but has oniy a small effect on the peak amplitude. 


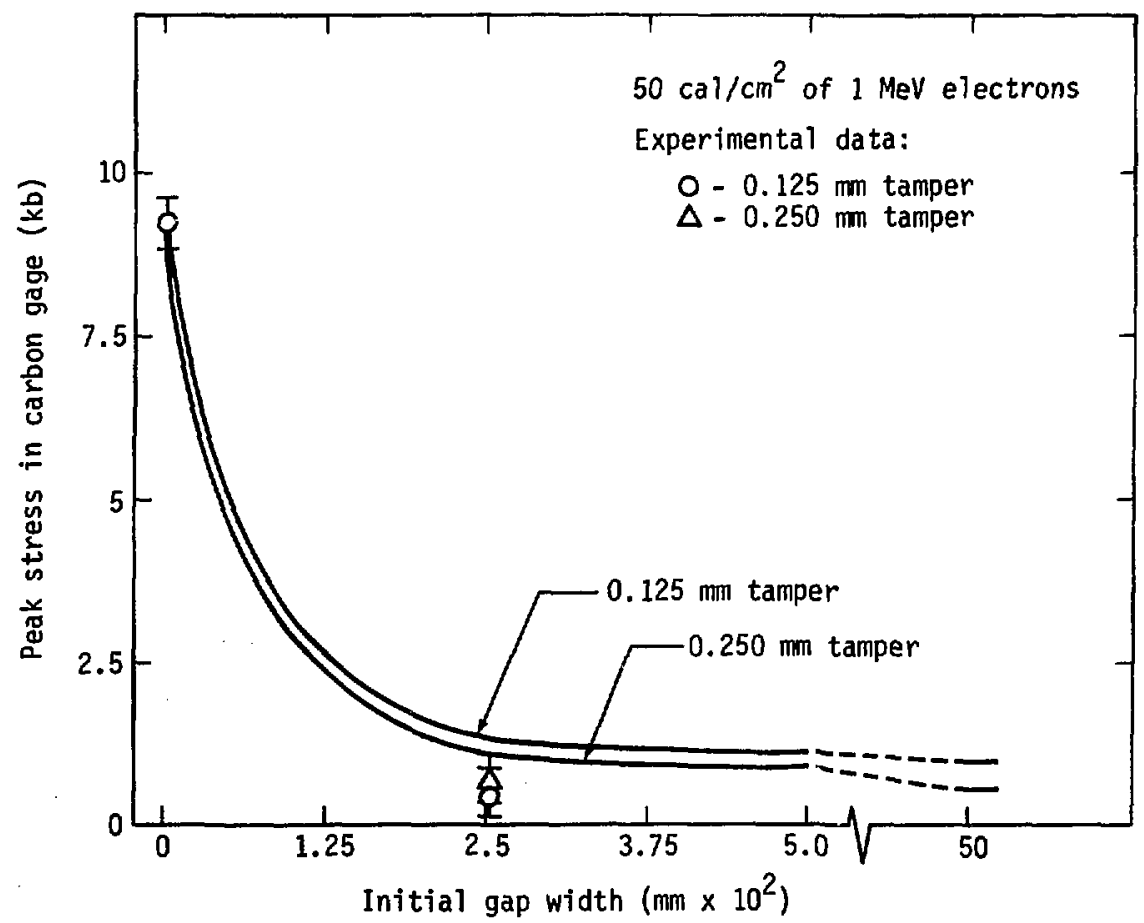

Figure 36. - Predicted levels of maximum carbon gage stress plotted versus initial gap size for the thin (0.025 $\mathrm{mm})$ lead targets. The measured peak stresses from the carbon gage are plotted as individual points. 


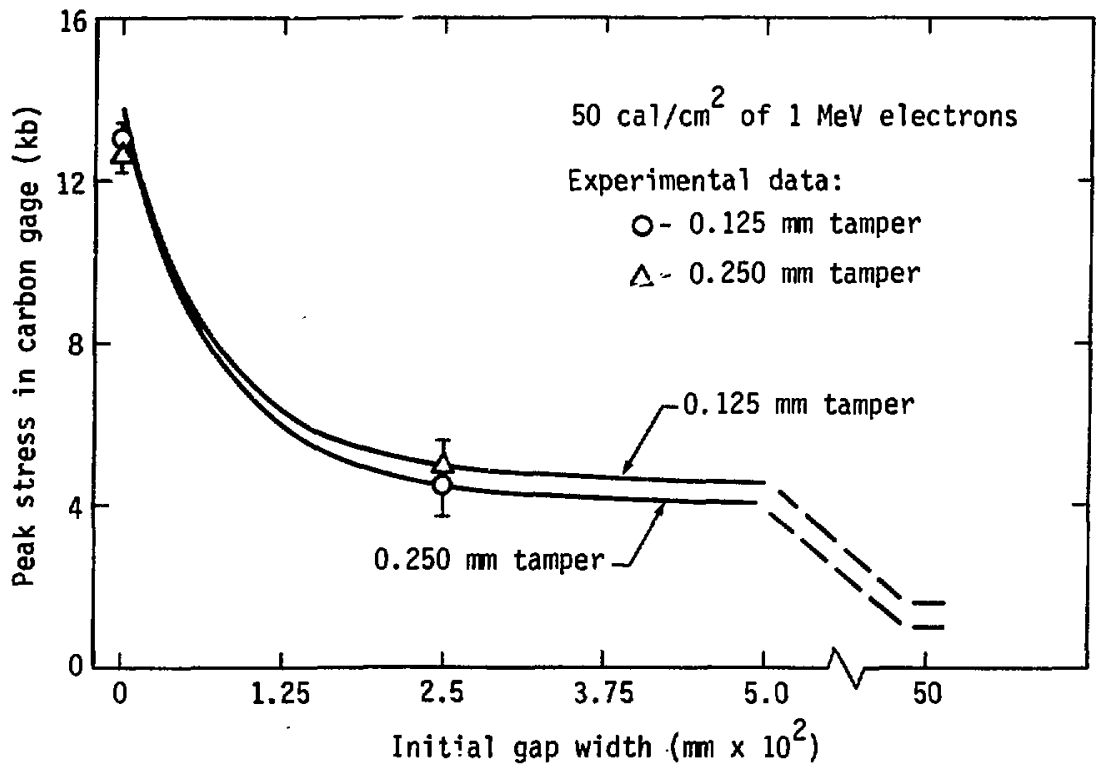

Figure 37. - Predicted levels of maximum carbon gage stress plotted versus initial gap size for the thick $(0.05 \mathrm{~mm})$ lead targets. The measured peak stresses from the carbon gage are plotted as individual points. 


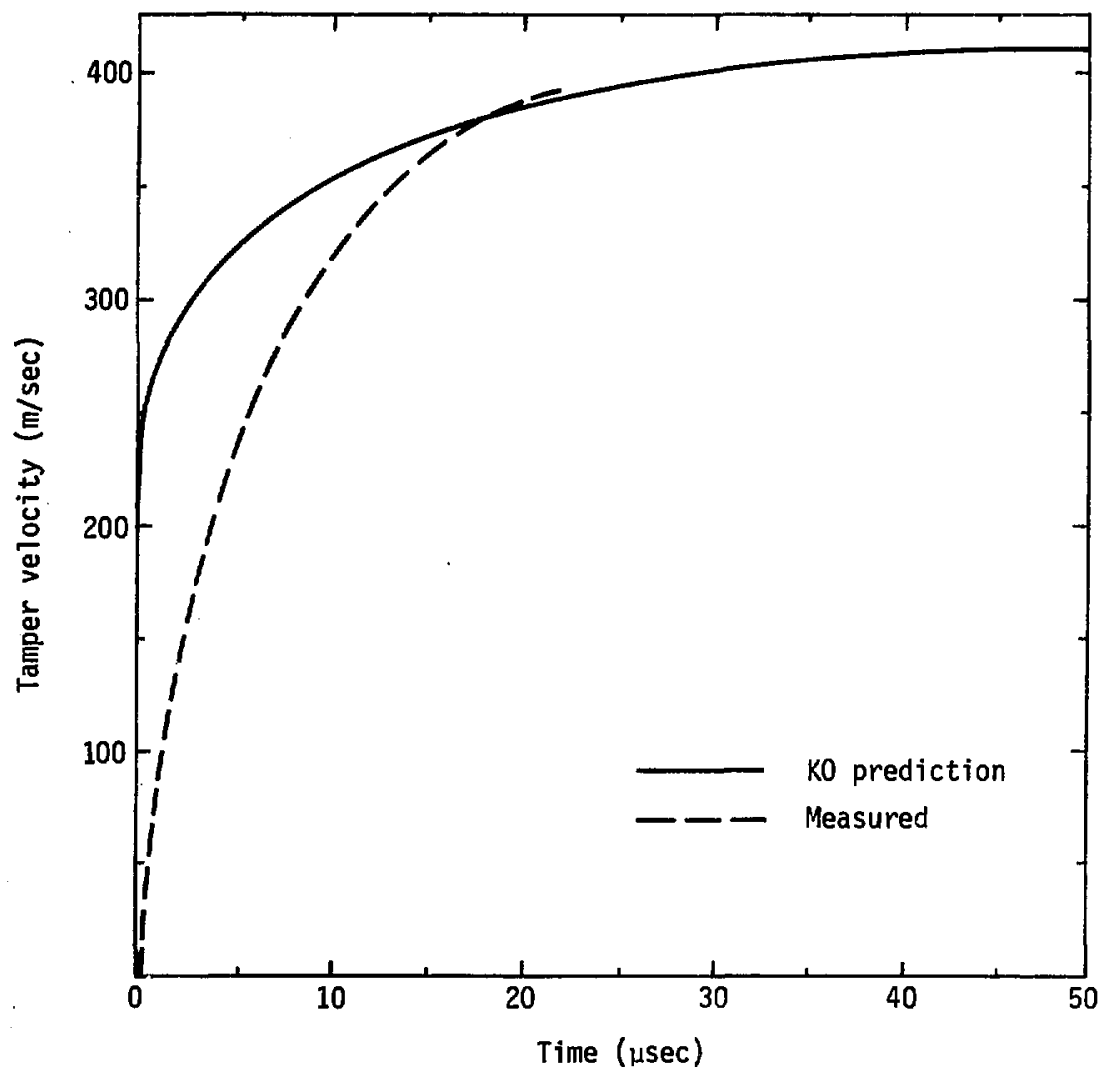

Figure 38. - Predicted tamper velocily plotted versus time. The initial Jump in velocity occurs as tamper velocity plotted versus time for the thick $(0.05 \mathrm{~mm})$ lead targets with a thick $(0.25 \mathrm{~mm})$ tamper. 


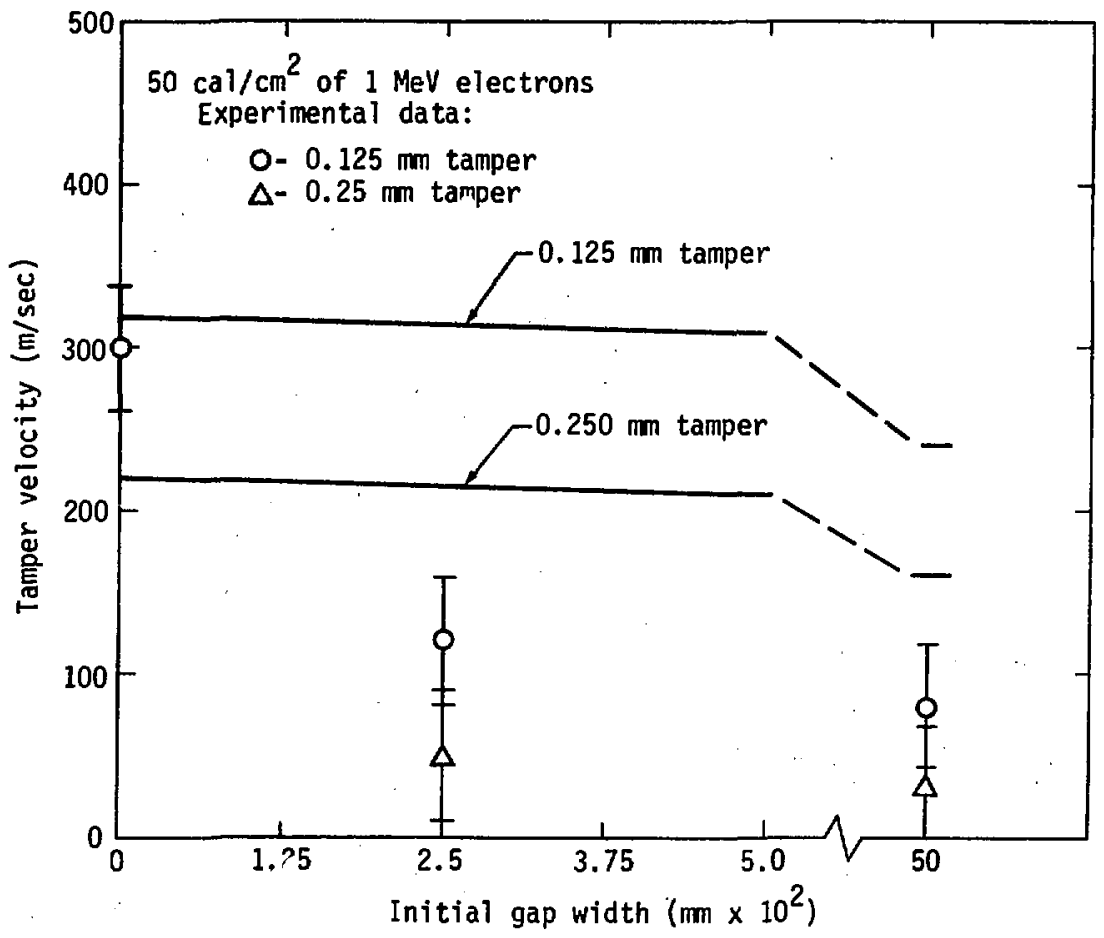

Figure 39. - Predicted tamper velocity plotted versus initial gap size for the thin $(0.025 \mathrm{~mm})$ lead targets. The measured velocities (averaged over the entire tamper) are plotted as individual points. 


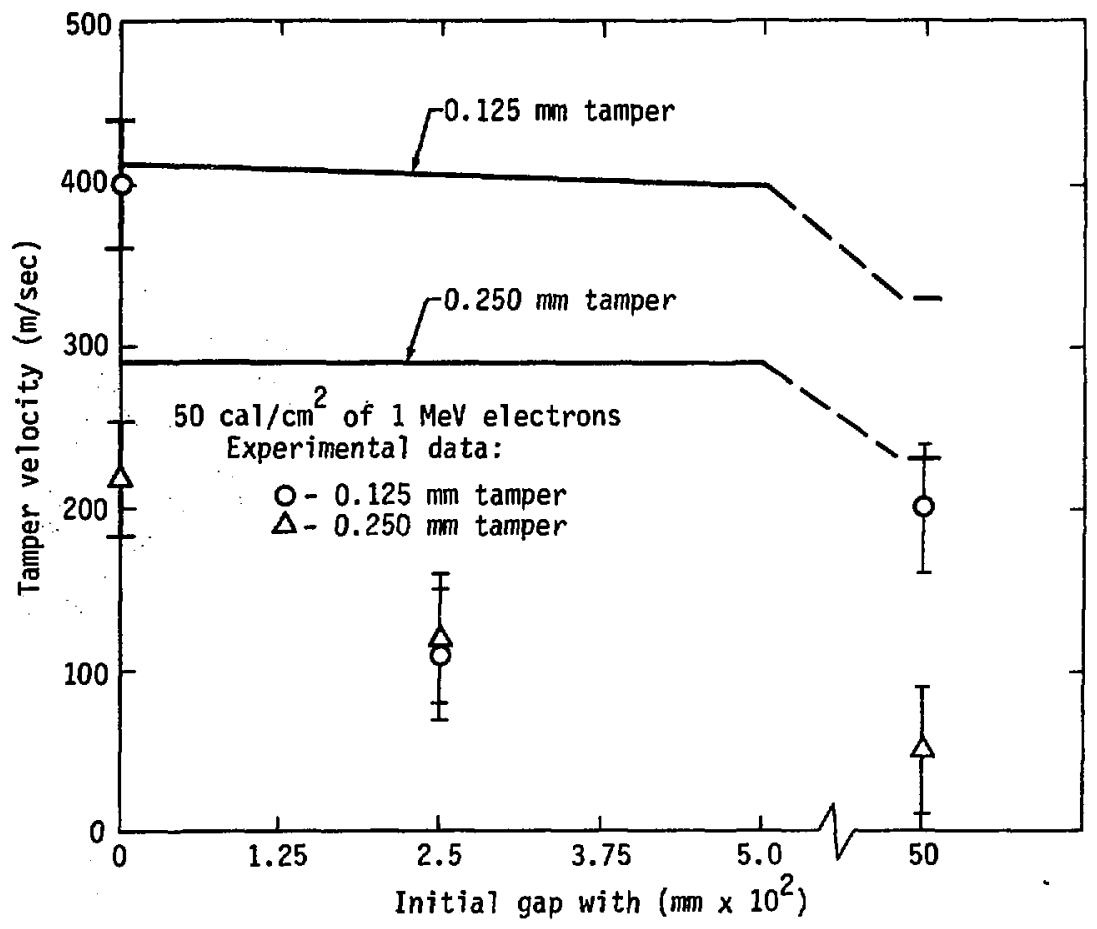

Figure 40. - Predicted tamper velocity plotled versus initial gap size lor the thick $(0.05 \mathrm{~mm})$ lead targets. The measured velocities (averaged over the entire tamper) are plotted as individual points. 


\section{DISCUSSION OF EXPERIMENTAL AND PREDICTED RESULTS}

Sections IV AND $V$ of this report detail the techniques and results of the experimental and analytical phases of this investigation. In this section experimental results are compared with analytical predictions and the areas of agreement and difference discussed. The stresses produced in the fused quartz buffer layer as measured by the carbon stress gage were presented in Figures 36 and 37 and will be treated first. A discussion of the titanium tamper velocity predictions and data as shown in Figures 39 and 40 will follow. Concluding this section will be consideration of the limitations of the analytical model with discussion of heat transfer to the tamper, the effects of liquid and vapor separation, and the GRAY EOS.

\section{COMPARISON OF PREDICTED AND MEASURED STRESS iN THE CARBON GAGE}

The stress measurements for the experiments with no gaps agree with the predicted values within about $10 \%$ (Figures 36 and 37 ). With small gaps the agreement was within a kilobar, but the level of stress was on the order of a kilubar so the percentage accuracy was not as good (about $50 \%)$. The stress gage data does. agree quite well with the predicted trend which shows the generated stress dropping very quickly as small gaps are introduced into the experiment assembly. This is true for botr the thin and thick lead targets and the light and heavy tampers. 
The experimerital data for the large gap case $(0.5 \mathrm{~mm})$ are not valid. The reason for this is that the electrons that pass through the lead target deposit energy in a thin surface layer of the quartz buffer plate. This deposition causes a small (about 0.3 kilobar) stress wave to propogate in the quartz buffer layer. For the zero and narrow gap cases, this small stress wave is overtaken by the much larger stress pulse resulting from the lead expansion. In the large gap tests the impact of the lead on the buffer is delayed nearly a full microsecond after the deposition is complete. This delay gives the smali precursor stress time to reflect and produce tensile stresses in the carbon gage laminations. This small tension destroys the integrity of the carbon gage and makes later readings, even compressions, unreliable.

EFFECT of INITIAL GAP - It can readily be scen in Figures 36 and 37 that the initial gap is much more impcrtant than the thickness (mass) of the tamping layer. Some expansion of the lead target occurred during the 50 nanosecond heating period. The amount of expansion (relaxation of the stresses) that could take place during the heating phase was affected by the initiel gaps in the assembly. If the lead were confined between infinite impedance tamper and buffer layers, constart volume rating would occur and the pressure would increase to $130 \mathrm{ki}$ lobars. A stress wave will transit $0.025 \mathrm{~mm}$ of lead in 12.5 nanoseconds; so if there were no barriers, considerable expansion would occur during the 50 nanosecond heatlng phase. The KovSPALL program predictions indicate that without tamping the expansion during the first 50 nanoseconds results in a relative volume $\left(\mathrm{V} / \mathrm{r}_{0}\right)$ of 2.0 . Instead of $130 \mathrm{kilobars}$ the average 
pressure is then 0.25 kilobars . about $1 \%$ of the internal (pressure) energy of the material has been converted to kinelic en irgy.

The bonded (zero gap) experiments provide a tamping effect throughout the 50 nanosecond heating phase. The effect is less than total tamping due to some titanium motion and quartz motion. The kOVSPALL program predictions for the thick lead targets indicate that, at the end of heating, expansion had decreased the pressure in the le:t to 18 kilobars et a relative volume of 1.25 . The 18 kilobars of pressure when transmitted into and propagated through the fused quartz to the carbon gage is reduced to 1.4 kilobars hy the impedance mismatch.. This is in good agreement with the $13 \mathrm{kjlobar}$ measured value.

Higher pressures are measured with the thick lead targets even though the thicker lead layer reduces the average energy depositon through the lead. The thin lead bonded larget cxperiments give stress mesurements of $9.5 \mathrm{ki}$ lobars as compared to the $1: 3 \mathrm{kilobars}$ observed in a thick target test. This occurs because a thin lead specimen can expand more quickly due to its thinner dimension. The lead layer itself provides some effective tampirg by preventing the stress relief waves from reaching the central portion of the lead instantaneously.

The small gap case fapproximately $0.025 \mathrm{~mm}$ on each side of the lead) provides enough room for unconfined volume expansion to a relative volume of 2.0 for the thick lead targets and 3.0 for the thin targets. This means that the lead will appear to be completely untamped during the heating phase, and the internal pressure will drop to an average of 
about 0.25 kilobar before tamping effects occur. Both the measured and predicted stresses are much higher than the average 0.25 kilobar which indicates that some of the kinetic energy in the lead is converted to laternal energy as it impacts the fused quartz. Figure 41 shows the predicted pressure versus time for the zone of lead ad;acent to the buffer plate. As the lead expands across the gap, the pressure drops and velocity increases. When the lead impacts the buffer, the pressure rises but does not reach the peak that is produced when the gap is removed.

The measurements validate the predictions for the small gap case. For both thicknesses of tamper and both sizes of lead target, the measured stress is much lower than the measured stress for zero gap runs. The agreement between measurement and prediction is excellent for the thick lead case at about 5 kilobars. The prediction for the thin lead case is about 1.25 kilobars as compared to the measured values of approximately $0.75 \mathrm{kilobar}$. When the gap size is the same for the thin and thick target cases, the thin lead undergoes a greater relative volume increase prior to impact. This additional expansion requires more time and, as will be discussed in detail later in this section, deviation of the two-phase fluid from the hydrodynamic model limits the model's ability to predict the material behavior for larger expansions. Even so, the experiment validates the trend of the prediction for a thin lead target. 


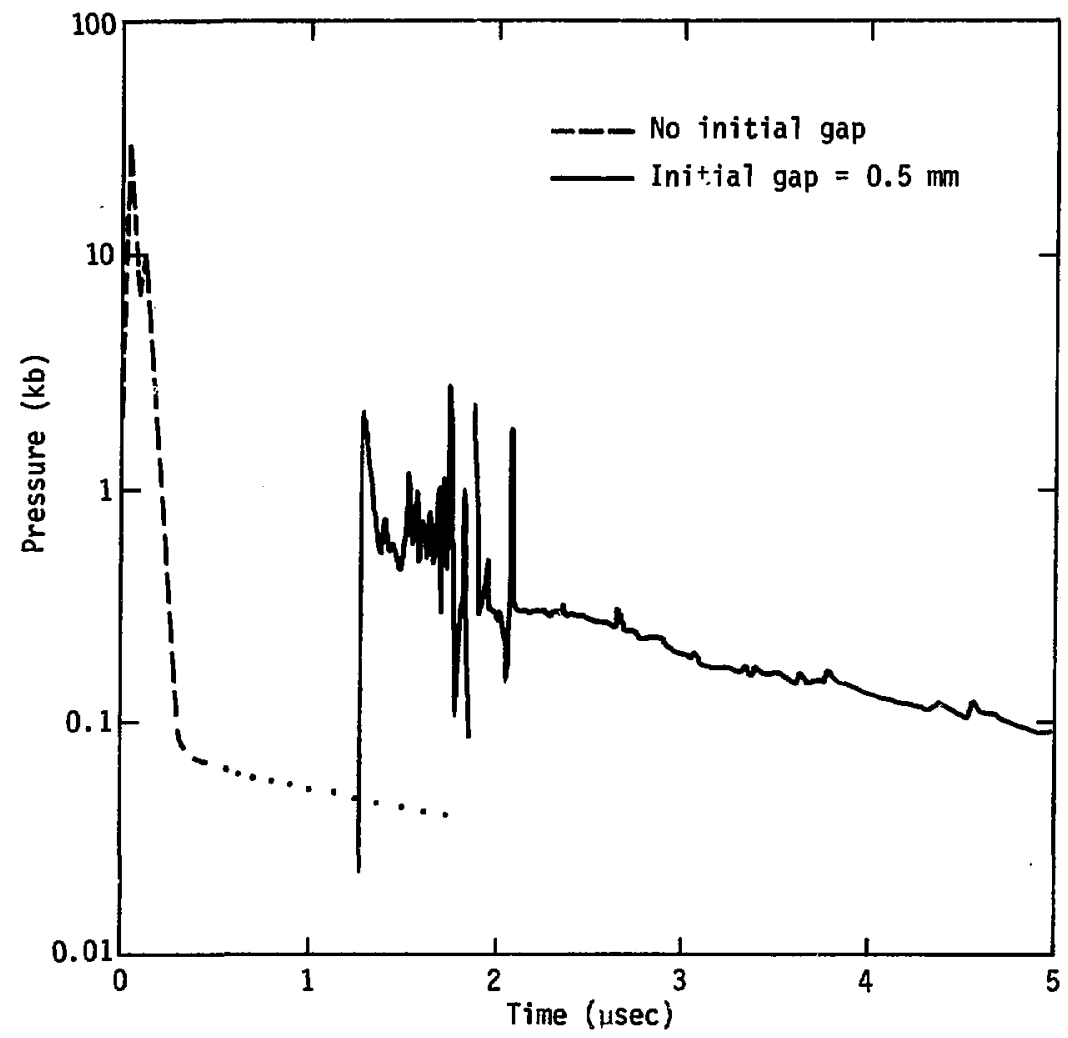

Figure 41. - The predicted pressure variations in the lead target layer adjacent to the quartz buffer plate plotted versus time. 
EFFECT of VARIATIONS IN TOTAL FLUENCE - The error bars on the experimental data points reflect a fluence variation of plus or minus 10\%. The peak stress is relatively insensitive to changes in total fluence. A higher fluence causes the lead to expand more rapidly and with more kinetic energy. This expansion decreases the effective tamping which has the effect of mitigating the increase in stress. A less than normal fluence allows the tamper to have a greater effect, thereby increasing the effective confinement and hence keeping the stress from decreasing as much as might be anticipated.

EFFECT OF THE SPATIAL DISTRIBUTION OF FLUENCE - Spatial non-uniformity of the fluence is potentially a serious problem as it introduces two dimensional effects. As was mentioned in the discussion of the electron beam in Section II, for a typical run the fluence on the center-line could be as much as $30 \%$ higher than the average while that at the corners was $30 \%$ less than the average. This will result in temperature and stress gradients in the lateral direction which will cause lateral stress waves.

Figure $42 a$ is a much simplified diagram of what an initial non-uniformity in axial stress at the quartz surface would approach in the time it takes the axial stress to be transmitted through the quartz buffer layer. The initial distribution was taken to be composed of three regions in which the axial stress levels corresponded to $1.3,1.0$, and 0.7 times the one dimensional average value produced by the expanding lead. Figure $42 b$ is a "characteristic" plot of the time 


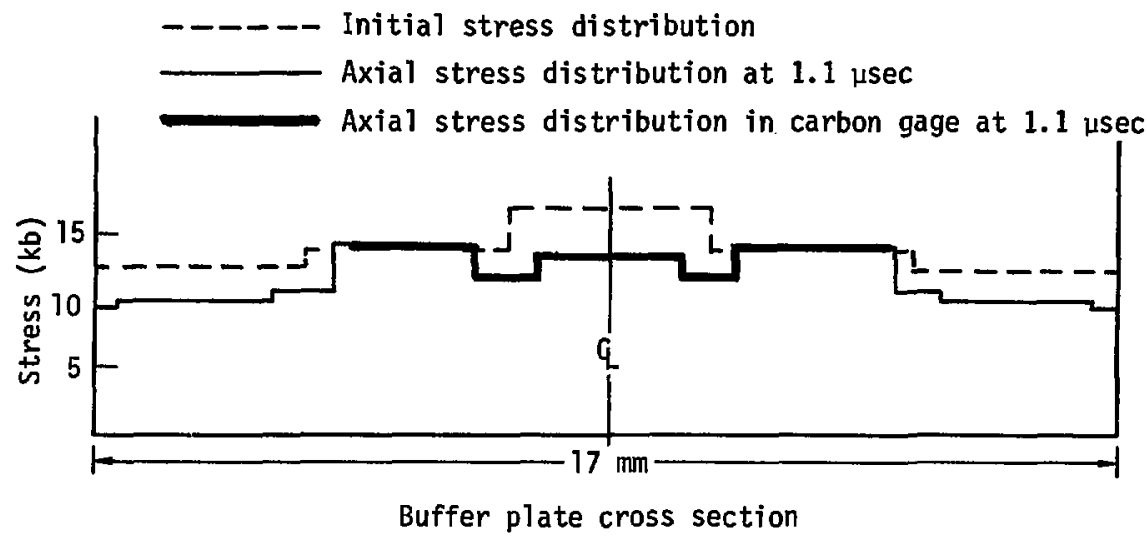

Figure 4Ra. - Initial stress distribution and resulting distribution of the compressive wave as it reaches the carbon gage location.

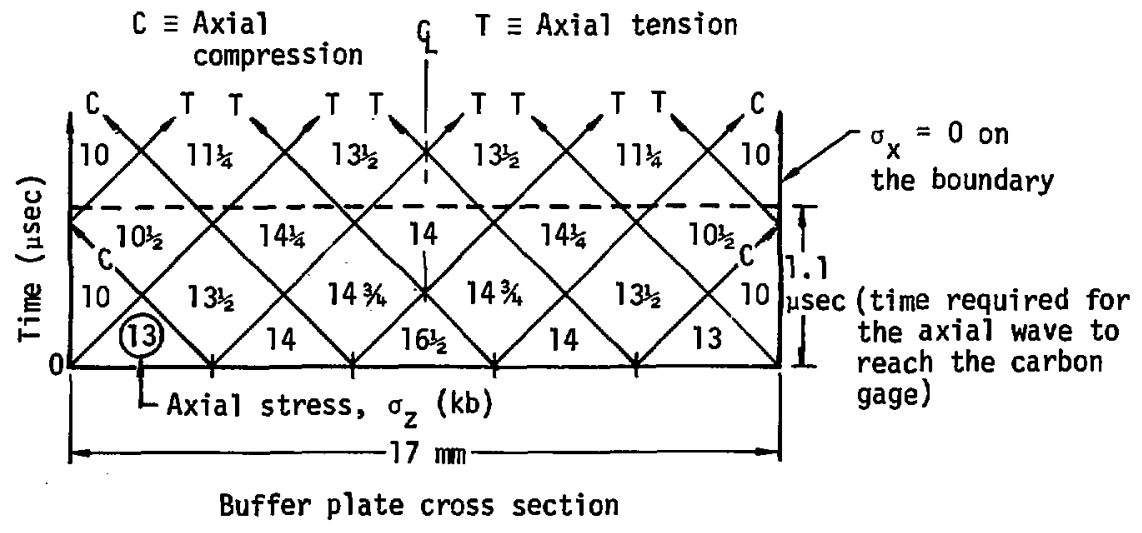

Figure 42b. - Graphical solution for estimating the lateral relief of the stress wave in the lused quartz buffer plate. 
versus position for the lateral stress waves developed from the initial distribution. A shear velocity of $3.75 \mathrm{~km} / \mathrm{sec}$ was taken for the fused quartz. The effect of the lateral relaxation on the axial stress distribution was evaluated from the changes indicated in Figure $42 b$ at an elapsed time of 1.1 microseconds (transit time for the axial stress wave). The result is shown by the solid line in Figure 42a. Notice that the average value for the stress intersecting the carbon gage is 14 kilobars, which is very close to the $14.2 \mathrm{kilobars}$ corresponding to the "average" fluence. Although this is a very simplistic description of the actual process, it can be seen that the lateral relaxation of the stress wave makes the use of the spatial average of the incident fluence an adequate approximation.

\section{COMPARISON OF MEASURED AND PREDICTED TAMPER VELOCITIES}

The agreement between observed and calculaled tamper velocities is within $10 \%$ for the zero gap-thin target condition (Fjgures 39 and 40 ). This agreement occurs at $t$ imes beyond 30 microseconds when the tamper velocity is approaching its asymptotic value. The measured velocities for the two experiments with $0.125 \mathrm{~mm}$ tampers and no initial gaps were within $10 \%$ of the calculated values. Only one data point for a bonded $0.25 \mathrm{~mm}$ tamper was obtained and it is $25 \%$ below the predicted level of $290 \mathrm{~m} / \mathrm{sec}$. However, for $f$ inite gaps, the analytical simulation definitely and consistently overpredicts the experimental results by a factor of 2 or more. 
It is interesting to consider the partition of momentum and energy in this process. The energy initially deposited in the lead is subsequently redistributed in the layers (zones) of material in the experiment. An investigation of where KOVSPALL predicts the energy to be stored can give clues to explaining the differences between experiment and prediction. Figures 43 and 44 shiow predictions of the energy transfer from the lead to its surroundings as a function of $t$ ime. Figure 43 is for a lightly tamped zero-gap run while Figure 44 is for a large gap and heavy tamping. For the heavily tamped no-gap example. approximately $10 \%$ of the energy deposited in the lead is transferred to the tamper as kinetic energy in six microseconds. Half of this or $5 \%$ of the deposited energy is transferred during the 50 nanosecond heating $t$ ime. The acceleration of the tamper is very rapid; four-fifths of the energy transferred is added to the tamper in less than one microsecond. In the lightly tamped and large gap case the predicted energy transfer from the lead to the tamper amounts to only about $3 \%$ of the energy added to the lead by the electron beam. This is only one third of the $10 \%$ transferred in the experiments with no gaps. At 50 microseconds for the heavily tamped thick lead experiment KOVSPALL predicts velocities of 290 and $230 \mathrm{~m} / \mathrm{sec}$ for the zero and large gap cases which means that in the zero-gap case, the tamper recieves 1.6 times the energy given to the tamper in the large-gap case.

EFFECT OF INITIAL GAP - The KOVSPALL program predictions are very insensitive to gap size (Figures 39 and 40). The calculated tamper velocity with 0.5 mm gaps is only $20-25 \%$ below the velocity calculated 


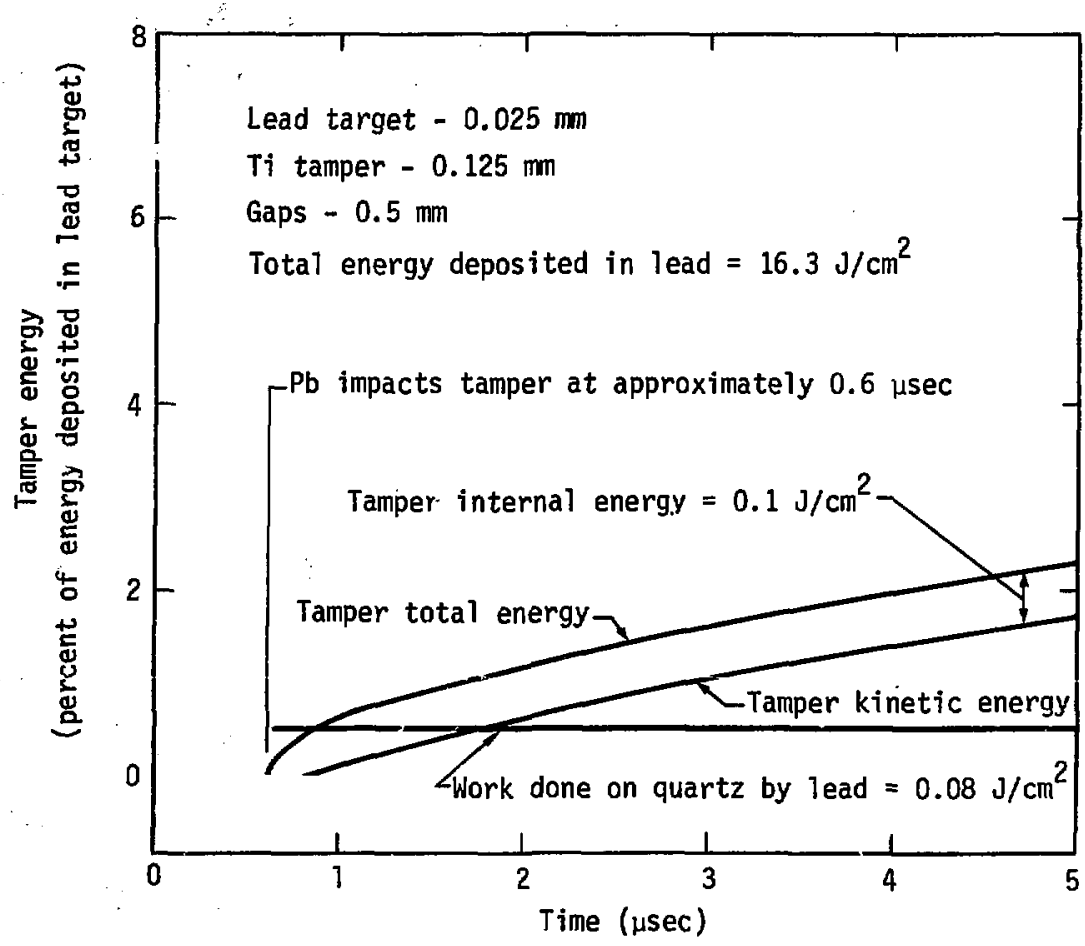

Figure 43. - Predictions of the energy transferred from the lead target to the tamper and buffer layers for a lightly tamped case. 


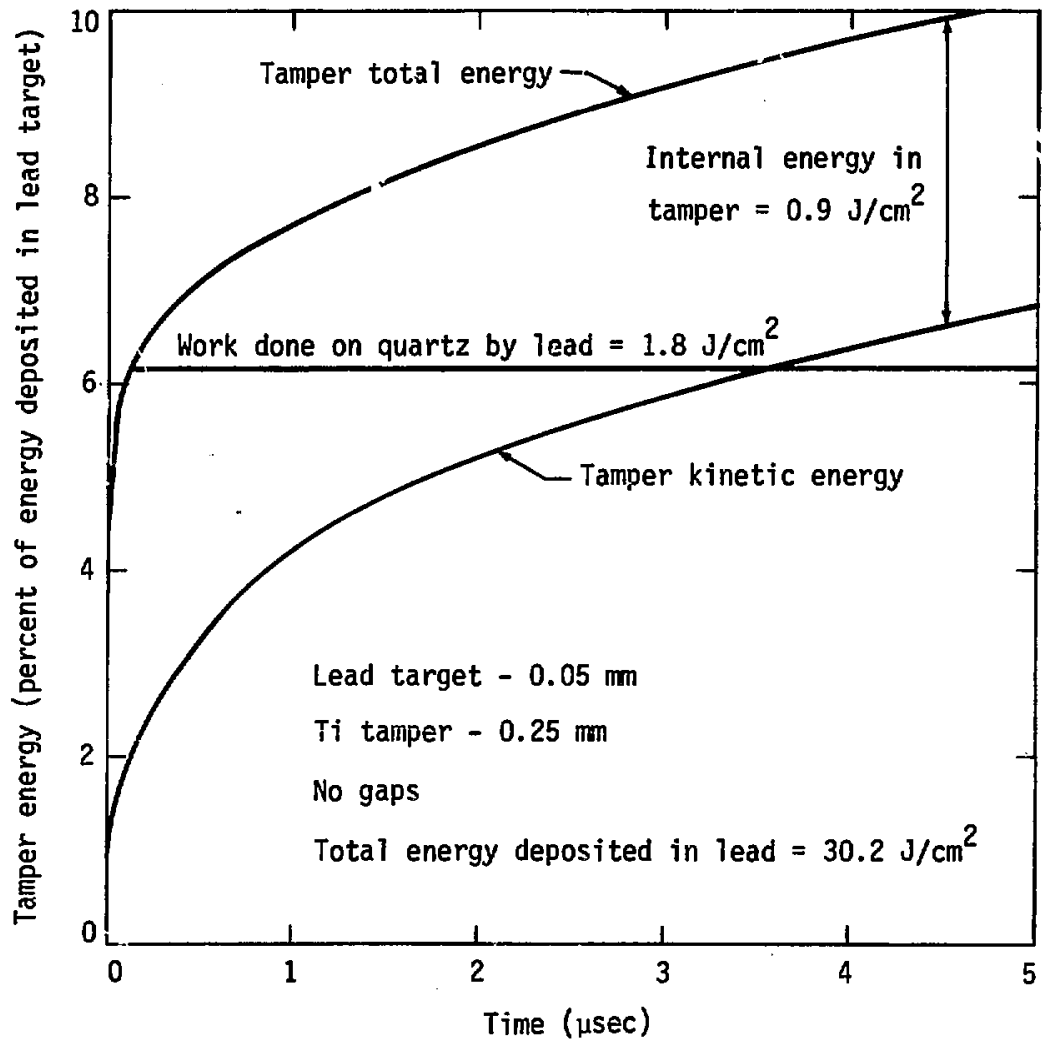

Figure 44. - Prediction of the energy transferred from the lead target to the tamper and buffer for a heavily iamped case. 
for the zero gap case. This is true for all four combinations of lead and titanium thicknesses. The measured velocities, as presented in Figures 39 and 40 , are close to predictions for the zero gap case, but drop significantly for even a small initial gap. Because of difficulty in controlling experimental variables, the error bars on the velocity méasurements are relatively large; however, the trend of significantly decreased velocily with the introduction of even a very small gap is clear.

It can be assumed that the initial tamper acceleration in the zero gap experiments is very high for the same reasons that are used to explain the high stresses produced in the fused quartz buffer. Specifically, the lead does work on the tamper from the start of the heating process when the very high pressures are produced in the lead. The experiments verify that the conversion of lead internal energy to tamper kinelic energy occurs as predicted. With gaps, the pressurc in the zone of lead that will impact and push the tamper decreases to a very low value before it contacts the tamper. This can be seen in Figure 45. The time integral. of this pressure (see Figure 46) after impact is the tamper momentum, which is proportional to the tamper velocity. The KOVSPALL program indicates that the peak pressure for the case with gaps is much lower and that the momentum increases much more slowly. However, at tens of microseconds, the asymptotic momentum is essentially the same for both cases due to the longer pulse that occurs with gaps. The stress measurements tend to confirm that the stress level at impect is approximately as predicted, and so it follows that 


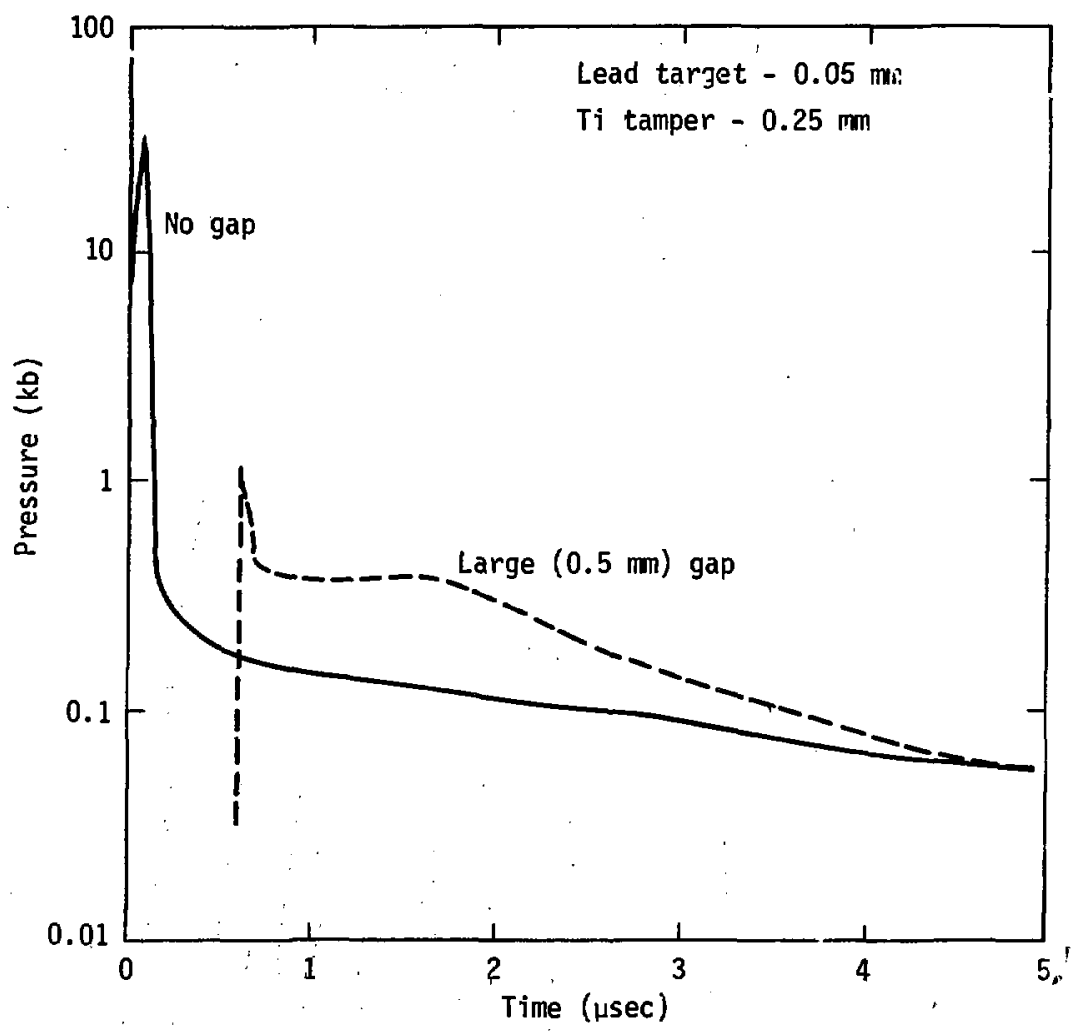

Figuie 45. - Predicted pressure in the lead target layer adjacent to the litanium tamper as a function of $t$ ime. 


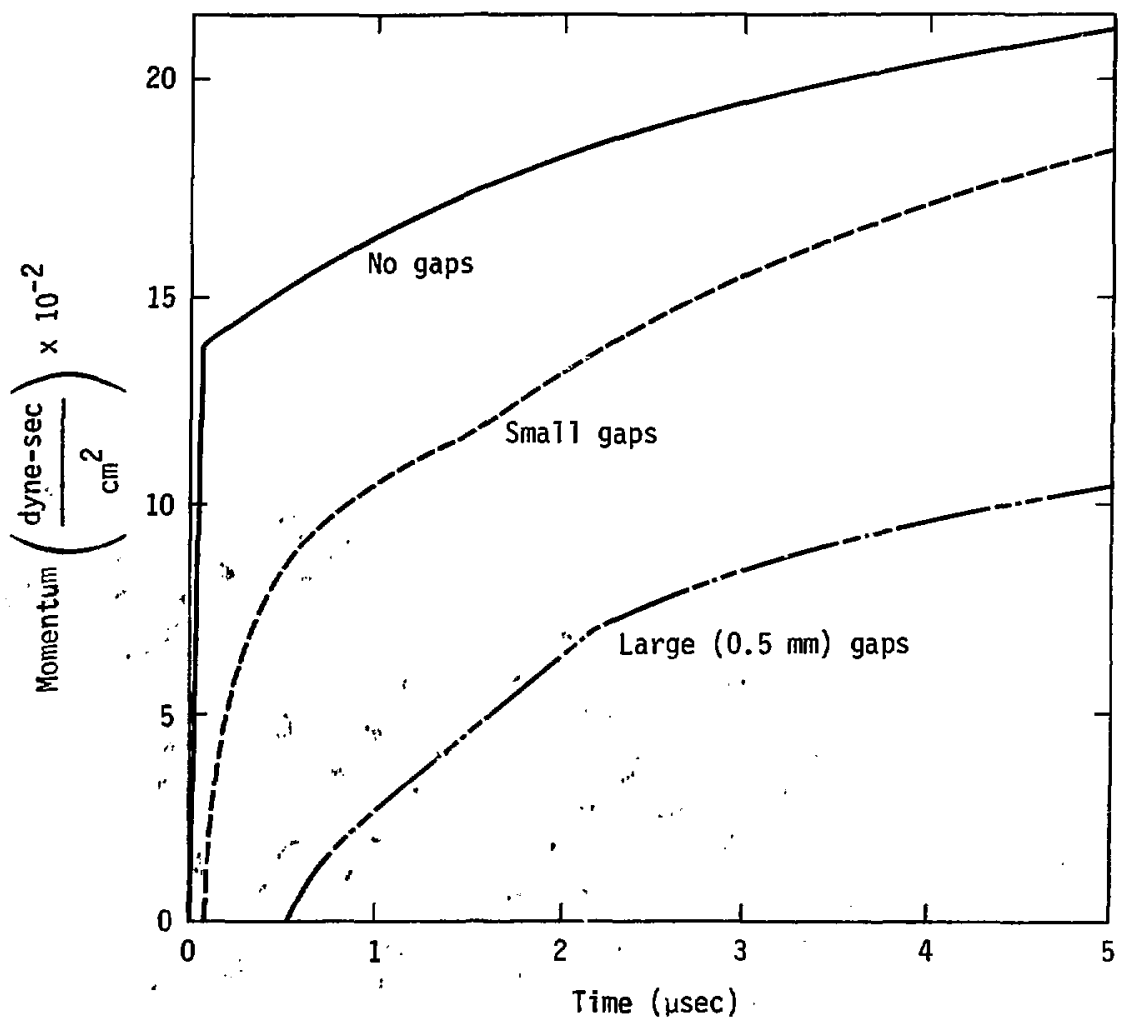

Figuré 46. - Momentum delivered to the tamper by the lead. Calculated by integrating the pressure time history in the lead layer adjacent to the tamper while the lead is in contact with the tamper. 
since the observed tamper velocity is low, the pressure pulse duration must be short. Some explanations for this will be proposed in the section on the applicability of the analytical model.

EFFECT OF VARIATIONS IN THE TOTAL FLUENCE - Ten percent fluctuations in the total fluence were found to change the predicted tamper velocities by only $15 \%$. These $15 \%$ variations are smaller than the accuracy to which the tamper velocities could be measured. Hence it is concluded that the difference between the predicted and measured tamper velocities could not be attributable to total fiuence varialion.

EFFECT OF THE SPATIAL DISTRIBUTION CF FLUENCE - The energy deposited at the axial center-line of the target is $30 \%$ higher than the fiuente averaged over the three $\mathrm{cm}^{2}$ area. This is shown in the results section to give a curved shape to the tampar as it is accelerated by the expanding lead. A KOVSPALL program calculation shows that the iamper velocity will increase $35 \%$ with a $30 \%$ increase in fluence. It also is shown in the results section that the strength of the tamper is insignificant at these high pressures and short $t$ imes. The peak tamper velocity observed in the experiments was $25 \%-50 \%$ higher than the average which is in agreement with these calculations.

\section{APPLICABILITY OF ANALYTICAL PREDICTIONS}

It has beer pointed out that there is good agreement between predicted and measured stresses. However, agreement between the experimentally determined and predicted tamper velocities is good only 
for the zero gap case. With finite gaps the measured velocities are significantly below predicted values. The question that therefore needs to be addressed is, "Why does the prediction technique significantly overestimate the momentum transferred to the tamping layer when other phases of the analysis seem to be valid?"

Possible answers to this question will be considered in this section and will include: 1) two-dimensional effects not treated by the analytical model, 2) the effect of heat transfer from the lead to the titanium tamper, 3) the problems associated with the use of a Lagrangian reference (rame for the liquid-vapor phase, and 4) the validity of the GRAY EOS (particularly in the liquid-vapor region).

LIMITATIONS OF A ONE- DIMENSIONAL MODEL - Although a more accurate value for the average stress in the carbon gage might he obtained from a two-dimensional analysis, this would require a more precise knowledge of the energy deposition than it was possible to measure. The finite size of the sarget means that the response will involve components of force and moticn in the lateral as well as axial directions. The edge reflections of stress waves, and the bowing of the tamping layer are examples of two dimensional effects in this experiment. It has already been pointed out that the carbon gage response is proportional to the everage stress over the area of the gage element. The same conclusion can be made regarding the tamper velocity calculation. The agreement between the bowed tamper shape and the fluence distribution is an indication that the effects are strongly one dimensional. 
Turning to a consideration of the two dimensional effects in the lead target, it is noted that the expanded lead has a very low density and hence a signjficantly decreased sound speed which tends to inhibit the propagation of disturbances. This decrease in the sound speed has the effect of suppressing lateral expansion of the lead layer. This was demonstrated in tests preliminary to this project where a lead-tamper assembly without a confining fixture was heated witr an electron beam (see Figure 47). As can be noted very little lateral expansion takes place, even in the absence of confining walls. The only significant lateral motion is the splash from the tamper and buffer plate. Hence one can conclude thiat the propagation or existence of $2-D$ phenemena in the liquid-vapor region is minimal. Further evidence of this slow vapor response is the fact that it takes 2.5 micreseconds for the pressure pulse caused by the lead impact on the tamper to reach the mass center of the lead layer in the experiment with large gaps. It is thus concluded that a multi-dimensional analysis would not yield sufficiently improved agreement between measurement and prediction of the results to warrant its inclusion here.

HEAT TRANSFER FROM THE LEAD TO THE TAMPER - Heat transfer in microsecond time scales is commonly ignored. In this experiment, however, there are two reasons for considering heat transfer: 1) the temperature differential is very high (on the order of $1200^{\circ} \mathrm{K}$ ) and 2) the kinetic energy actually transferred to the tamper is only a few joules per $\mathrm{cm}^{2}$. Therefore an estimate of the amount of heat transferred and a discussion of its effect on the experiment will be made in this section. 


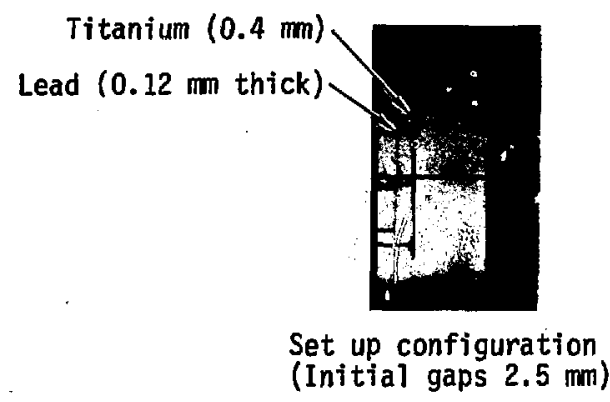

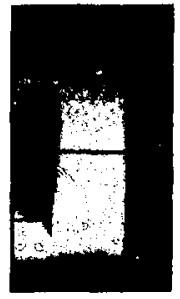

0-1 usec

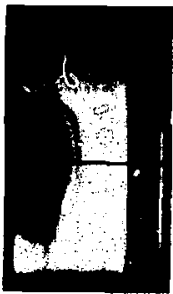

$20 \mu \mathrm{sec}$

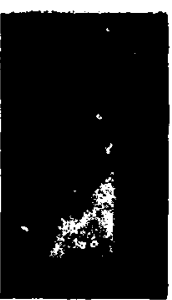

40 usec

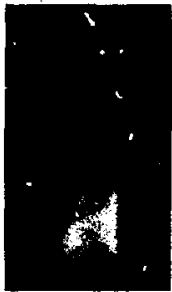

$60 \mu s e c$

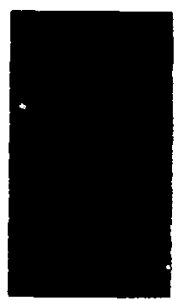

80 usec

Figure 47. - Framing camera sequence of liquid-vapor lead expanding against. a titanium tamper, but without a containment cavity. 
The heat transfer from the lead to the titanium can be approximated by assuming that two semi-infinite bodies at different temperatures are suddenly brought into contact with no interface resistance 27 . If the internal temperatures are uniform and of magnitudes $T_{1}$ and $T_{2}$, the interface temperature is found to change instantaneously to a constant value $T_{s}$; and the heat rate from one body to the other is given by:

$$
w=w_{1}=w_{2}=\frac{k_{1}\left(T_{1}-T_{s}\right)}{\sqrt{\pi \alpha_{1} t}}=\frac{k_{2}\left(T_{s}-T_{2}\right)}{\sqrt{\pi \alpha_{2} t}}[\mathrm{~J} / \mathrm{sec}]
$$

where

$$
\begin{aligned}
& \mathrm{k}=\text { thermal conductivity }\left[\mathrm{J} /\left(\mathrm{sec} \mathrm{cm}{ }^{\circ} \mathrm{K}\right)\right] \\
& \alpha=\text { thermal diffusivity }\left[\mathrm{cm}^{2} / \mathrm{sec}\right]
\end{aligned}
$$

Solving for the interface temperature yields:

$$
T_{s}=\frac{T_{1} \frac{k_{1}}{\sqrt{\alpha_{1}}}+T_{2} \frac{k_{2}}{\sqrt{a_{2}}}}{\frac{k_{1}}{\sqrt{\alpha_{1}}}+\frac{k_{2}}{\sqrt{\alpha_{2}}}}
$$

The total amount of heat transferred per unit area in $t$ ime $t$ is given by

$$
W(t)=\int_{0}^{t} w(t) d t=\frac{2}{\sqrt{\pi}}\left(T_{3}-T_{2}\right) \frac{k_{2}}{\sqrt{a_{2}}} t^{0.5}
$$

The GRAY EOS indictes that the equilibrium temperature for isentropically expanded lead is about $3,500^{\circ} \mathrm{K}$ at relative volumes near twenty. The temperature of the titanium is estimated to be $1,400^{\circ} \mathrm{K}$ 
based on an energy deposition of $150 \mathrm{cal} / \mathrm{gm}$ (see Figure 10) and a specific heat capacity of $0.586 \mathrm{~J} /\left(\mathrm{gm}^{\circ} \mathrm{K}\right)$. Using the following values for the conductivity and diffusivity for lead and titanium

$\begin{array}{llll} & \underline{\mathrm{Pb}} & \underline{\mathrm{Ti}} & \\ \mathrm{k} & 0.37 & 0.071 & \mathrm{~J} /\left(\mathrm{cm}-\mathrm{sec}-{ }^{\circ} \mathrm{K}\right) \\ \alpha & 0.25 & 0.027 & \mathrm{~cm}^{2} / \mathrm{sec}\end{array}$

the surface temperature, $T_{S}$, is calculated to be approximately $2600^{\circ} \mathrm{K}$. Inserting this into Equation (25) yields the following magnitudes of energy transferred to the tamper from the lead.

$\begin{array}{cc}\text { TIME }(\mu \text { sec }) & W(t)(\mathrm{J} / \\ 0.1 & 0.19 \\ 1.0 & 0.61 \\ 3.0 & 1.1 \\ 6.0 & 1.4 \\ 30 . & 3.4\end{array}$

These are very rough estimates, possibly overestimating the magnitude of the heat transfer, because of the difficulty in establishing accurate thermal properties for the lead. The amount of heat transferred to the quartz buffer and walls of the cavity is neglected because the heat transfer rate to quartz is only $35 \%$ of the rate to the titanium. The accumulated heat transfer to the tamper at 30 microseçonds is about $10 \%$ of the energy deposited in the thick lead targets by the electron beam, and about $20 \%$ of the energy deposited in the thin targets: To add confidence in this calculation it was noted that the processes. involved in this experiment were similar to the acceleration of a light weight projectile in a gun barrel. In this case energy is supplied by the chemical energy in a powder propellent rather 
than the electron beam. Measurements have shown ${ }^{28}$ that the heat transfer to a gun barrel during firing is on the order of $10 \%$ of the energy released. This supports the conclusion that the heat transfer does not deplete the total energy in the lead to a significant extent.

SEPARATION OF THE LIQUID AND VAPOR PHASES - For the level of energy deposited during expansion, the lead sample will undergo a flashing process, i.e. the "instantaneous" formation of vapor. There is very limited information about how this actually occurs. lllustrative results of experiments by Miyatake et. al. ${ }^{2 \theta}$ on flash evaporation of water by rapid decrease in ambient pressure are shown in Figure 48 . Il is interesting to note in those photographs that the first formation of vapor is bubbles randomly distributed in the liquid and fully formed in 0.17 seconds. This is at the fairly modest superheat of $3.88^{\circ} \mathrm{C}$.

In the electron beam experiments with a fraction of $50 \%$ by weight of vapor ultimately being formed, it is thought that the vapor bubbles will tend to coalesce into a continuous phase and the liquid to form into droplets. There can be little doubt that this conlescing takes place: the time that is required for bubble formation to become noticable is not well known.

It can be argued that the presence of an initial gap in the experiment could affect the amount and more importantly the rate of phase separation. Even with $0.025 \mathrm{~mm}$ gaps there is space for the thick lead target to double its volume before it encounters resistance. With the large gaps the thin lead samples can undergo a 40-fold expansion 


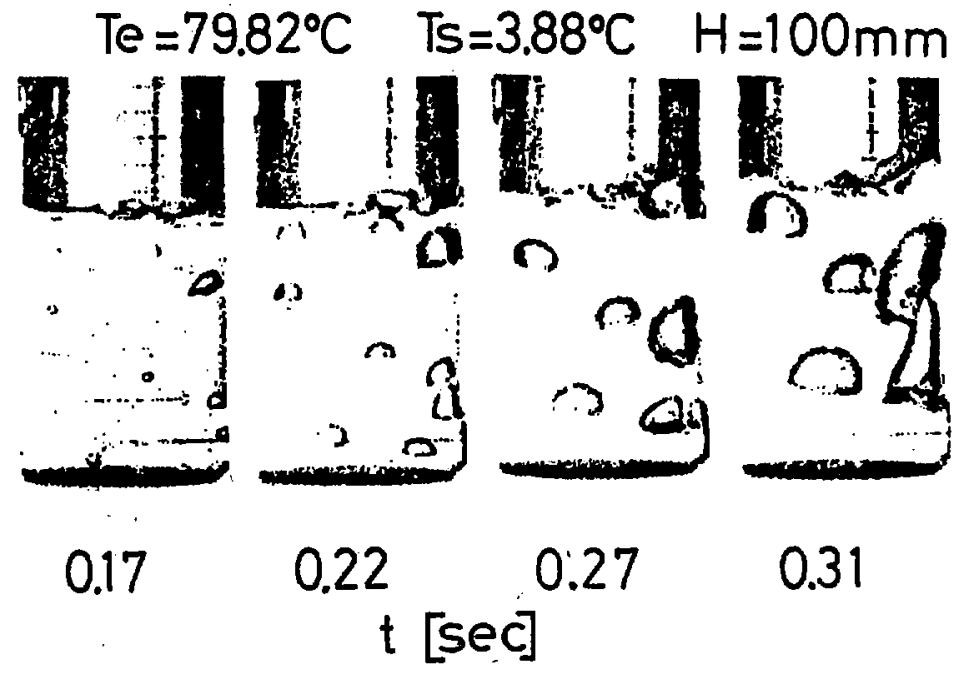

Figure 48. - Photographs of water undergoing a flash evaporation process: The pressure above the water surface was decreased to $100 \mathrm{~mm}$ $\mathrm{Hg}$ at which pressure the superheat amounted to $3.88^{\circ} \mathrm{C}$. (from Reference 29) 
before impacting the tamper and buffer layers. Motion of the tamper can add another factor of 5-10 during the velocity measurement period. It would be unrealistic to assume that the liquid and vapor stay uniformly mixed during a 400-fold increase in relative volume. The predictions and observations establish that the experiments with no initial gap result in a prompt acceleration of the tamper. This means that this configuration is less affected by the time dependent phase separation phenomenon which can only begin to have an effect after there is a several fold expansion of the two-phase mixture.

The hydrocode analysis technique assumes that each of the 25 zones that make up the lead layer consists of a homogeneous mixture of liquid and vapor. The EOS used to compute material properties will return a unique pressure given a specific volume and energy. It is inherent in the formulation of the hydrocode equations that the zone is considered homogeneous. To consider it otherwise would really be considering multiple zones. As currently written the code does not make any attempt to rezone the two-phase material with respect to phase separation. If there is no implementation of mechenical separation within a zone, then there can be no transfer of a particular phase to a neighbor zone.

An estimate of the viscous drag on the liquid by the vapor indicates that the droplets would not be accelerated to velocities required for the two phases to remain uniformily mixed. The drag coefficient on a sphere at velocities corresponding to a Mach number near 0.5 is on the order of $0.5^{30}$. At $3000^{\circ} \mathrm{K}$ the ratio of saturated 
vapor to saturated liquid density for lead is $0.005^{31}$. Inserting these values into Equation (26) , the viscous acceleration of spherical droplets :

$$
\begin{aligned}
a_{d}=\frac{F_{D}}{M_{1}} & =\frac{C_{D} A_{d} \frac{\rho_{v} u_{v}^{2}}{2}}{\left(4 \pi r^{3} \rho_{1}\right) / 3} \\
& =\frac{(0.5) \pi r^{2} \rho_{v} u_{v}^{2}}{\left(4 \pi r^{3} \rho_{1}\right) / 3} \\
& =0.4 \cdot \frac{\rho_{v} \cdot u_{v}}{\rho_{1} r}
\end{aligned}
$$

Solving for the droplet radius, $r$, shows that $r$ mst be less than five micrometers for the liquid to reach $40 \%$ of the vapor velocity in five microseconds. Because the lead is a superheated liquid at the end of energy deposition and the vapor is formed because due to expansion into the two phase region, the existence of such small liquid drops is very unlikely. Hence the conclusion that the liquid will not be accelerated by the vapor is valid.

Separation of the two phases of heated material will lead to substantial differences in the predicted behavior, particularly at the later times. It has already been indicated that the pressure distribution in the region containing the lead is highly non-uniform. The lead stagnates at high pressures against the buffer and expands to lower pressures against the accelerating tamper. The large expansions near the tamper result in lower acoustic velocities and hence longer 
time constants associated with achieving equilibrium. If the material near the tamper is predominantly vapor with its relatively low density, the acoustic velocity of the layers near the tamper will will be even lower than that calculated in the hydrocode for a "uniform mix". Also in this case the energy lost by heat iransfer to the tamper is more appropriately compared to the energy content in the lead layers near the. Lamper, hot to the total energy in the. lead.

The total lead energy was 15 to $30 \mathrm{~J} / \mathrm{cm}^{2}$ which means, because there are 25 equally spaced zones in the lead layer, that 1.2 to $2.4 \mathrm{~J} / \mathrm{cm}^{2}$ are contained in the two lead layers adjacent to the tamper. The heat loss: calculations show that it is reasonable to consider losses on the order of a joule per $\mathrm{cm}^{2}$ which would cut the energy in those layers by nearly $50 \%$. The expanded nature of the lead and a preponderance of vapor would inhibit the transfer of heat energy from the interior layers of lead. The pressure of the lead adjacent to the tamper will then fall below the levels predicted by the hydrocode analysis and the tamper will not reach the calculated velocities.

An additional effect is that the vapor molecules which are accelerated toward the tamper come in contact with the relatively cold surface where they lose their kinetic energy, stick to the titanium and condense. Without a liquid portion near the iamper to continue producing vapor by boiling, the local pressure will drop. The drop in local pressure will prevent the tamper from achieving the velocities calculated assuming a uniform mixture. 
The ocurrence of separation was also used by Benson ${ }^{32}$ to explain the results of similar experiments. His Experiments consisted of a thin layer of gold restrained between two graphite tamping layers. Both tamping layers were free to move as the heated gold expanded. The tampers had difierent thicknesses and hence different masses. The velocities of the the tamping layers when scaled by dividing by the effective mass of the tamper should be consistent. It was pointed out by Benson that the scaling masses that gave the best results were the tamper masses alone without any gold assumed to be moving with the tamper. Those results suggested that most of the liquid-phase material 'remained unaccelerated during the vapor expansion.

The tamper velocities calculated in the hydrocode analysis represent maximum credible velocities for homogeneous material without heat transfer. The very real effects of nucleation and separation and growth of vapor pockets, and the removal of energy by lieat transfer while considered only approximately in this report can be seen to account for the differences between experimental observations and analytical predictions.

GRAY EOS - Betause the expanded lead is either liquid or vapor, the van der "Waals portion of the GRAY EOS accounts for virtually all the predicted lead response for the experiments with initial gaps. The join from the scaling law portion to the van der Waals portion is accomplished at a specific volume of 1.24 . With even a $0.025 \mathrm{~mm}$ gap the relative volume is three before impact on the tamper occurs. The entire 
expanded region in the EOS is specified by two parameters; the volume exclusion coefficient, $V_{b}$, and the attractive potential coefficient, $a_{Y}$. The value of $V_{b}$ is determined by the "hard sphere" diameter and $a_{Y}$ is a function of the cohesive energy of the molecule. These coefficients are used in equation 27 tc return the pressure, $p$, as a function of volume and temperature in the expanded region of the GRAY EOS surface.

$$
\begin{aligned}
& P=P_{0}-a_{Y} / V \\
& a_{Y}=-E_{0} / V_{0} \\
& \mathrm{P}_{0}=\frac{R T}{\mathrm{~V}} \cdot \frac{1+\nu+\nu^{2}-\nu^{3}}{(1-\nu)^{3}} \\
& \nu=\frac{\pi N \sigma^{3}}{6 \mathrm{~V}}=\frac{\mathrm{V}_{\mathrm{b}}}{\mathrm{V}} \\
& \mathrm{E}_{0}=\text { cohes } \mathrm{ive} \text { energy } \\
& \sigma=\text { hard spheie diameter }
\end{aligned}
$$

The fact that the entire liquid-vapor region is modeled with an equation using oniy two parameters is an indication that there will be areas on the EOS surface where the fit is not particularly good. The GRAY model predicts $2.08 \mathrm{~kb}$ for the critical pressure of lead but experimental data indicates that it is $0.86 \mathrm{~kb}^{31}$. As can be seen in Figure 33 the slope of the saturated liquid line is very steep in the region below $1 \mathrm{~kb}$. A shift of this boundary by only enough to change the pressure of the lead by 0.5 could make the predictions agree much more closely with experiment. In general it is entirely possible for the GRAY EOS to be in error by as much as a kilobar in the low pressure regime is. $^{33}$ 
An attempt was made to adjust $a_{Y}$ to get better agreement with the experimental data. Royce ${ }^{17}$. suggests varying $a_{Y}$ by as much as $30 \%$ if necessary to achieve closer agreement with experimental data. There was no physical reason for the adjustment as the initial value of aY equal to 49 was derived from the best available static data. Increasing the value of $a_{Y}$ by $30 \%$ decreased the two-phase equilibrium pressure for isentropically expanded lead. When the same starting point at $V_{0}$ and $5500^{\circ} \mathrm{K}$ was used, the expanding lead entered the two-phase region at 0.08 $\mathrm{kb}$ instead of $0.3 \mathrm{~kb}$ for a four-fold reduction. However, in modeling the experiment the resulting effect on tamper velocity was ro nearly as dramatic; the tamper velocity was reduced by only about $10 \%$. The expansion of the lead zones that impact the tamper is essentially "free" and when the lead stagnates against the tamper, pressures are near the same $0.5 \mathrm{~kb}$ to $1.0 \mathrm{~kb}$ seen in the nominal case. Increasing the value of $a_{Y}$ even more only resulted in numerical instability in the Eos calculation. Changes in the other coefficient, $v_{b}$, also showed very little effect on the tamper motion results.

Even if these coefficient adjustments had been succesful, an explanation of the reasons for the improvenent before using the new values for any other experimental process would be required. There would be no confidence that the modeling itself was any more accurate with the new values wi thout contributing evidence from experiments using other areas of the expanded EOS surface. Thus these experiments must be considered inconclusive in regard to determining the validity of the 
GRAY EOS to correstly calculate the proper equ-librium pressure in the low pressure two-phase region. 


\section{CONCLUSIONS AND RECOMMENDATIONS}

\section{CONCLUSIONS}

A pulsed electron beam can be applied for the investigation of the dynamic response of materials suddenly heated into the supercritical regime. Careful consideration, however, must be given to the selection of experimental conditions so as to provide a repeatable and uniform electron pulse. Repeatability is achieved through appropriate choice of the pulsed electron beam machine components, Of these the selection of the anode-cathode configliration including the anode-cathode spacing is most important. Uniformity of deposition in the target specimen is a function of both the e-beam machine output and the experiment fixture assembly. A successful solution to these challenges was demonstrated in this study.

On the basis of results obtained, it is concluded that the peak stresses produced by a supercritical liquid expanding into the liquid-vapor region depend very strongly on the degree of confinement. The maximum stress produced in adjoining layers by the expanding liquid reached nine kilobars for the $0.025 \mathrm{~mm}$ lead specimens and 13 kilobars for the $0.05 \mathrm{~mm}$ specimens when the layers were in close contact. The peak stress drops rapidly with the presence of even small gaps to a level less than one kilobar for the $0.025 \mathrm{~mm}$ targets and about five kilobars for the $0.05 \mathrm{~mm}$ targets. Currently available one-dimensional hydrodynamic computer models are able to predict the peak stress to 
within a kilobar for the tegts with closely confined targets and with initial gaps.

It is: also concluded that the momentum transferred to the tamping layer is substantially dependent on the existence and size of initial gaps between the expanding liquid and the adjoining layers. When there were no gaps in the initial experiment configuration, the tamper velocity (momentum) measurements agreed with the predicted values within the experinental error. However, for even very small gaps, experimental data for tamper velocity are lower than predicted values by a actor of at least two to ihree. This trend was attributed primarily to phase separation effects which also mean that one-dimensional computer programs based on a fixed mass per element are not directly applicable to predicting momentum transfer when there are initial gaps.

Finally, these tests established that the tamper mass has little effect on the peak stress generated in the fused quartz. Increasing the tamper thickness by a factor of two changed the stress measured $w$ ith the carbon gage by only about $10 \%$. The momentum transfer to the tamper depends on the mass per unit area of the tamping material. The double thickness tamper was accelerated more slowly, but the velocity was not decreased by one half as might be expected. The slower expansion rate kept the cavity pressure somewhat higher in the heavy tamper case thereby of $f$ setting some of the effect of increased mass. 


\section{RECONOENDATIONS}

This study suggested the importance of phase separation and heat transfer during tlee expansion of a liquid into the liquid-vapor region. Effortg should be made to understand the phenomena of bubble nucleation and growth on the time scales and at the energy levels typical of these experiments. The possibilities of using a pulsed energy source such as an e-beam or laser to produce in-depth heating of a translucent target layer (e.g. Water) in which the bubble formation could be studied optically should be investigated. If individual bubbles cannot be resolved in time and space, an estimate of the liquid density might be obtained by projecting a low power laser through the liquid-vapor region. By not including the carbon stress gage, the area of the target could be reduced, making it easier to get the laser beam through the mixture. All array of photodiode detectors could return a time resolved signal of the spatial distribution of the two-phase mixture.

Analytical modeling of the phase separation process will be required for hydrocode calculations of momentum transfer. In addition to adding heat transfer to the calculation, a first step would be to specify the physical location of the different phases based on experimental data, retaining the basic Lagrangian coordinate system. A complete model. would have to include turbulence thus adding an entirely new set of problems. 
Additional effort should be made to improve the elertron beam pulse to obtain more uniform and repeatable energy deposition and to improve the tamper impulse measureing techniques. Calorimetry experiments could be devised that would quantify in real time the actual energy transferred to the tamper and buffer layers. A method for determining the temperature profile of the expanding mixture optically would aid in determining the actual heat transfer rate. 


\section{REFERENCES}

1. J. A. Maniscalco and L. L. Wood, Advanced Concepts in Fusion-Fission Hybrid Reactors, UCRL-75835, Lawrence Livermore Laboratory, Livermore, Calif., July 1974 .

2. S. Glasstone, The Effects of Nuclear Weapons, United States Atomic Energy Commision, February 1964.

3. D. A. Schauer, Thermal and Dynamic Effects in Electron Beam Welding Cavities, UCRL-52331, Lawrence Livermore Laboratory, Livermore Calif.. August 1977 .

4. R. T. Avery, Study of Rock Shat tering by Intense Bursts of Energetic Electrons, LEL-3019, Lawrence Berkeley Laboratory, Berkeley Calif.. May 1974.

5. J. F. Ready, Effects of High-Power Laser Radiation, Academic Press. New York, 1971.

6. L. W. Woodruff, W. H. Giedt, and J. L. Hesse, "Surface Melting, Spallation, and Stresses Induced in Metals by Pulsed Electron Beam. Heating", Journal of Applied Mechanics, Vol. 38 page 363, June 1971.

7. G. R. Gathers, J. W. Shaner, and D. A. Young, "An Improved Apparatus for Thermophysical Measurements on Liquid Metals Up to $8000 \mathrm{~K}$ ", Review of Scientific Instruments, Vol. 47 No. 4 page 471 , April 1976.

B. T. F. Wieskamp, L. W. Woodruff, and S. E. Starkey, The Nuclear Effects Simulation Test Facility, Lawrence Livermore Laboratory Internal Memorandum. September B, 1972.

9. F. R. Kovar, ETRANMS - A One-dimensional Monte Carlo Electron/Photon Transport Code for Multimaterial Targets, UCRL-51493. Lawrence Livermore Laboratory, Livermore, Calif., November 1973.

10. M. J. Berger and S. M. Seltzer, Electron and Photon Transport Programs, Reports 9836 \& 9837, National Bureau of Standards, June 1968 .

11. F. R. Kovar, Comparison of ETRAN Calculations with Experiments, UCRL-74517, Lawerence Livermore Laboratory, Livermore, Calif.. February 1973.

12. \%. J. Naumann, Carbon Stress Gage Handbook, CR 72-101, Fifects Technology, Inc., Santa Barbara, Calif.. October 1972. 
13. E. O. Williams, Operators Manual for Model 600-50-75 Manganin Pulse Power Supply, SLL-DR-70-148, Sandia Laboratories, Livermore, Calif.., January 1971 .

14. D. Karnopp and R. C. Rosenberg. Analysis and Simulation of Multiport Systems, The M.I.T. Press, Cambridge, Mass., 1968.

15. G. L. Goudreau, DTVIS2/POST2 Users Manuals, Lawrence Livermore Laboratory Internal Memorandum, October 18, 1973.

16. J. P. Woodruff, Kovec Users Manual, UCID-17306, Lawrence Liver̃more Laboratory, Livermore, Calif.., November 1976.

17. E. B. Royce, GRAY, A Three-Phase Equation of itate for Metals, UCRL-51121, Lawrence Livermore Laboratory, Livermore, Calif.. September 1971 .

18. S. L. Thompson and H. S. Lauson, Improvements in the CHART D Radiation-Hydrodynamic Code II: A Revised Program, SC-RR-710713, Sandia Laboratories, Albuquerque, N. M., February 1972.

19. M. L. Wilkens, Calculation of Elastic-Plastic Flow, UCRL-7322, Rev. I, Lawrence Livermore Laboratory, Livermore, Calif., January 1969.

20. Ya. B. Zel'dovich and Yu. P. Raizer, Physics of Shock Waves anu High Temperature Hydrodynamic Phenomena, Vol. II page 685, Acaciemic Press, New York, 1967.

21. R. Grover, "Liquid Metal Equation of State Based on Scaling", Journal of Chemical Physics, Vol. 55 No. 7 page 3435, October 1971.

22. D. A. Young and B. J. Adler, "Critical Point of Metals from the van der Waals Model", Physical Review, Vol. A3 page 36., January 1971.

23. D. J. Steinberg, Private Communication, September $19 ; 6$.

24. M. van Thiel, Compendizm of Shock Wave Data, UCRL-50108, Lawrence Livermore Laboratory, Livermore, Calif., June 1966.

25. L. H. Bakken and P. D. Anderson, An Equation-Of-State Handbook, SCL-DR-68-123, Sandia Laboratories, Livermore, Calif., January 1969.

26. D. A. Young, Modification of the GRAY Equation of State in the Liquid-Vapor Region, UCRL-51575, Lawrence Livermore Laboratory, Livermore, Calif ., April 1974.

27. L. R. Ingersol, O. J. Zobel, and A. C. Ingersol, Heat Conduction with Engineering. Geological and other Applications, page 90, University of Wisconsin Press, Madison, Wisc., 1954. 
28. W. H. Giedt, "The determination of Transient Temperatures and Heat Transfer at Gas-Metal Interface Applied to a $40 \mathrm{~mm}$ Gun Barrel". Jet Propulsion, Vol. 25 No. 4 page 182, April 1955.

29. 0. Miyatake, K. Murakami, Y. Kawata, and T. Fujii, "Fundamental Experiments with Flash Evaporation", Heat Transfer - Japanese Research, Vol. 2 No. 4 page 89, December 1973.

30. A. H. Shapiro, The Dynamics and Thermodymamics of COMPRESSIBLE FLUID FLOW. The Ronald Press Co., New York, 1953.

31. A. V. Grosse and A. D. Kirshenbaum, "The Temperature Range of Liquid Lead and Silver and an Estimate of their Critical Constants", Journal of Inorganic and Nuclear Chemistry, Vol. 24 page 739, November 1962.

32. D. A. Benson, "Vapor Properties of Gold at Exlreme Temperatures by Pulsed Electron-Beam Measurement": Journal of Applied Physics, Vol. 47 No. il page 4873 , November 1976.

33. D. A. Young, Private Communicatior, June 1977. 


\section{ACKNOWLEDGEMENTS}

I would like to acknowledge the valuable and essential support received from so many individuals during the course of this effort. I regret not being able to mention all of them here.

Dr. Lawrence Woodruff as mentor and committee member provided the suggestion and encouragement for this undertaking. His continued interest and insights were essential to all of this work.

Prof. Warren Giedt was the catalyst required for pulling all of the diverse parts of this effort into a completed project. His guidance and enthusiasm throughout all this investigation were invaluable. Prof. William Hoover and Dr. David Young provided valuable suggestions and guidance for the analytical EOS discussion.

Raymond Netherton served as resident inventor, designer, machinist, and operator during the experiments. His talents for high speed photography, his attention to detail, and his ability to create hardware to solve assembly and performance problems provided solutions at critical stages in the investigation. John Claar as operator of the electron-beam facility was an integral member of the experiment team. I would also like to thank Dr. Joseph Keller, Jack Deligans, Stan Starkey, and Dr. Hans Kruger for their encouragement and support.

My family deserves much credit for refraining from asking, except at annual intervals, "When will all this be finished?" It finally is. 


\section{APPENDIX A. - NOMENCLATURE}

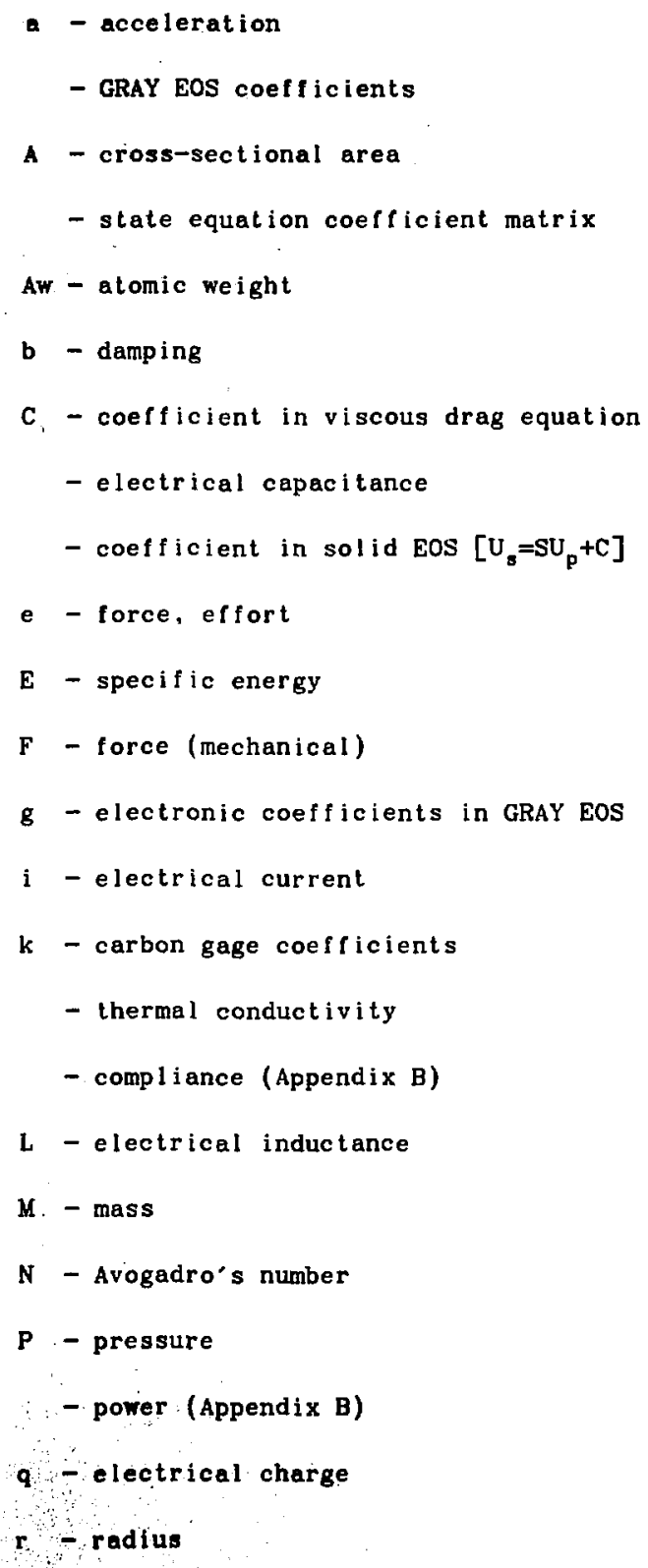




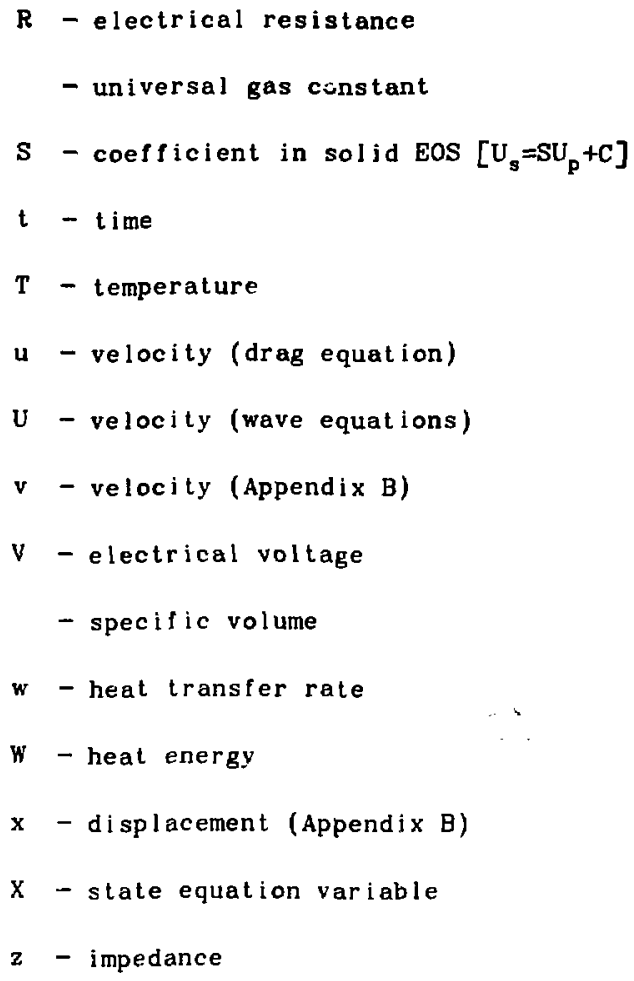




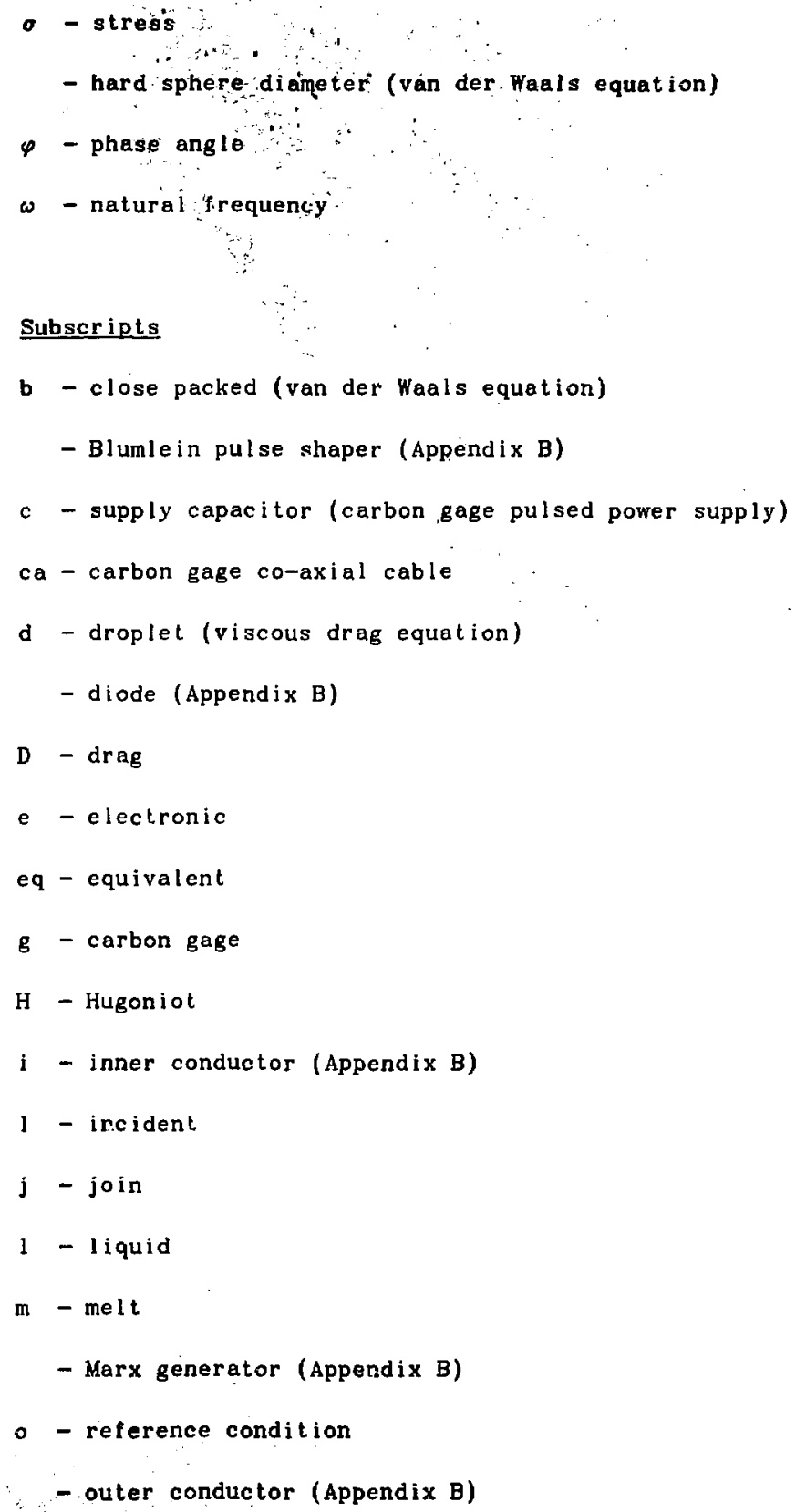




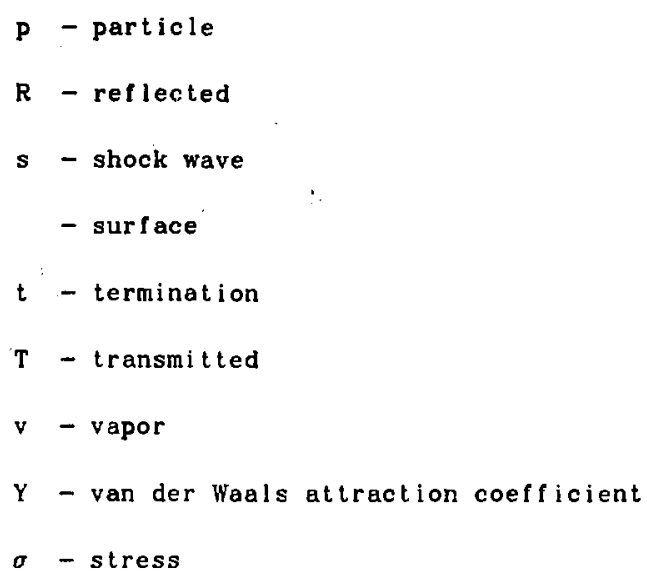

\section{Superseripts}

- impedance in terms of force per unit flow

\section{Prefix}

$\Delta$ - interval of change

Single underline - vector (nxi array)

Double underline - matrix (nxm array) 


\section{APPENDIX B.}

\section{A Description of the Dynamic Response of the Marx Generator}

and Pulse Shaper Using a Mechanically Equivalent System

The pulsed electron beam machine used for these experiments utilizes a Marx Generator to produce a $2.5 \times 10^{6}$ volt charge from a $100 \mathrm{kV}$ d.c. supply. A coasial concisetor holds the Marx generator charge until a diode with a field enission cathode is connected to the conductor. The coaxial conductor has very little inductance and discharges very rapidly providing the diode with a controlled width (shaped) pulse of energy. Another pulse shaping network utilizing a triaxial conductor called a Blumlein pulse shaper is available and can provide better pulse tailoring at higher diode impedances. The benefit of the triaxial configuration is that it allows the total voltage developed by the Marx generator to be applied to the diode, whereas in the coaxial conductor only half of the maximum voltage is across the diode during the pulse. For optimum performance the diode impedance must be nearly the same as the impedance of the coaxial conductor, while if the Blumlein network is used, the diode impedance must be near the sum of the impedances of the inner and outer conductor pairs of the triaxial conductor.

The Blumlein pulse shaper was used in an early phase of these experiments until electron pulse energy requirements made the coaxial arrangement necessary. The dynamic response of the Blumlein circuit is morecomplicated and difficult to visualize than that of the coaxial system and an equivalent dynamic model using mechanical elements is 
presented here. Figure Bl shows schenatic diagrams of the Blumlein network and its mechanical analog. Through most of Appendix $B$ explanations of the electrical and mechanical system responses will be done simultaneously in parallel columns of text beginning with a list of the equivalent properties and units of the two systems.

1. Basic Parameters and Units:

ELECTRICAL

$\mathrm{V}$ electrometive force [volts]

q charge [soulombs]

i current [amps]

$$
\mathrm{i}=\mathrm{dq} / \mathrm{dt}
$$

z'impedance [ohms]

$$
z^{\prime}=V / i
$$

C capacitance [farads]

$$
\mathrm{V}=\mathrm{q} / \mathrm{c}
$$

\section{MECHANICAL EQUIVALENT}

F force [newtons]

$\mathrm{x}$ displacement [meters]

$\mathrm{v}$ velocity [meters/sec]

$$
v=d x / d t
$$

$\mathrm{z}^{\prime}$ impedance $[\mathrm{N} /($ ineters/sec $)]$

$$
\mathrm{z}^{\prime}=\mathrm{F} / \mathrm{v}
$$

$1 / \mathrm{k}$ compliance [meters/N]

$$
F=k \cdot x
$$

capacitors and springs in parallel add

$$
\begin{array}{c|c}
C=C_{1}+C_{2}+\cdots+C_{n} & k=k_{1}+k_{2}+\cdots+k_{n} \\
& \text { capacitors and springs in series add reciprocally }
\end{array}
$$

$$
\frac{1}{c}=\frac{1}{c_{1}}+\frac{1}{c_{2}}+\cdots+\frac{1}{c_{n}}
$$

L inductance [henries]

$$
\begin{gathered}
\mathrm{l}=\mathrm{L} \cdot \mathrm{di} / \mathrm{dt} \\
\mathrm{R} \text { resistance (ohms) }
\end{gathered}
$$

$$
V=j \cdot R
$$

$$
\frac{1}{k}=\underset{k_{1}}{-}+\underset{k_{2}}{-1}+\cdots+\frac{1}{k_{n}}
$$

$M$ mass [ki lograms]

$$
F=M \cdot d v / d t
$$

b damping $[(\mathrm{N}-\mathrm{sec}) /$ meter $]$

$$
F=v \cdot b
$$




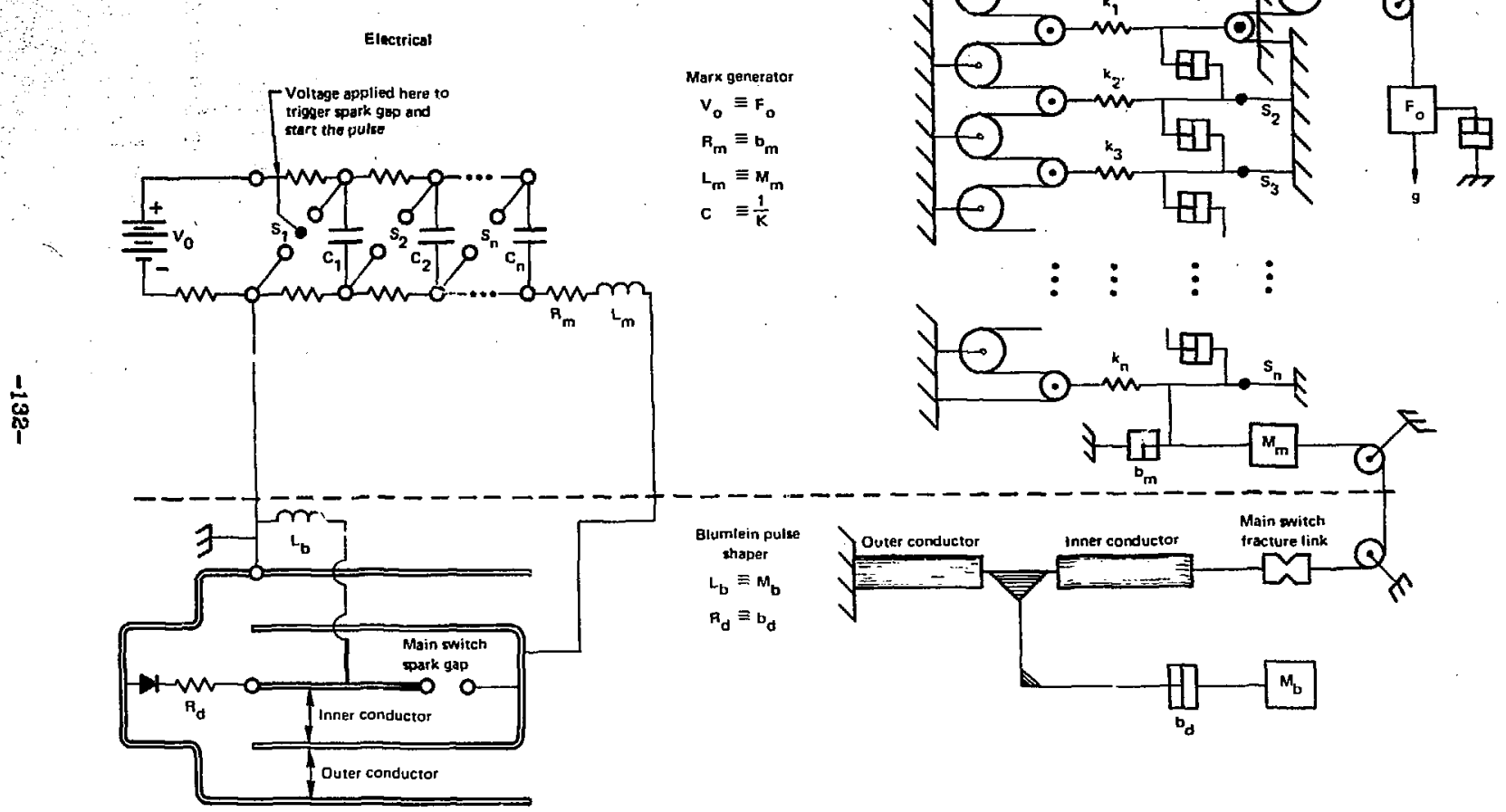

Figure B1. Electrical schematic of a Blumlein pulse shaper network and a mechanically equivalent linkage. 


\section{Operation:}

\section{A. Charging the Marx Generator}

\section{ELECTRICAL}

The de power supply charges

the capacitors in parallel to

voltage $V_{0}$. The charge supplied

by the power supply is $n \cdot\left(c \cdot v_{0}\right)$,

the charge required for $n$

capacitors connected in parallel.

When this charging sequence is

complete, each capacitor has been

charged to $v_{0}$ volts and no current

is flowing in the circuit.
MECHANICAL

As shown in Figure B1, a force, $F_{0}$, is applied to a cable ${ }^{\dagger}$ and pulley arrangement that extends each of the springs, $k_{i}$, by an emount $x_{0}$ equal to $F_{0} / k$. The displacement of $F_{0}$ is $n \cdot\left(F_{0} / k\right)$, the total displacement of n springs connected in series. When equilibrium is at tained, the system will remain at rest.

B. Triggering the Marx Generator

When the Marx charging

sequence is complete, the first

spark gap $\left(S_{1}\right)$ is closed by

overloading it with a vollage

spike from the trigger circuit.

The closed spark gap has very low

resistance and transfers the

potential from $C_{1}$ to spark gap $S_{2}$.
When the springs are streched and the energy storage phase is complete, cable $S_{1}$ is cut at the point indicated in Figure $\mathrm{BL}$. The force in $k_{1}$ is transferred to cable $S_{2}$ through the damper connecting $k_{1}$ and $k_{2}$. This overloads cable $S_{2}$ and causes it

t - In parts of this analysis cables will act in compression. While this is not physically possible, the actuel linkages required would be too cumber some to include. 
The overload on $S_{2}$ causes it to break down and conduct

transferring the combined

potentials of $C_{1}$ and $c_{2}$ to $S_{3}$.

- This process closes all the spark

gap switches and the Marx

generator is connected to the

Blumlein pulse shaper. The

capacitors are connected in series

which presents a voltage potential

of $n \cdot v_{0}$ to the pulse shaper input. to break, transterring the load of

$k_{1}$ and $k_{2}$ on to the cable at $k_{3}$.

Eventually all of the cables

$\left(S_{1}-S_{n}\right)$ are broken and the springs

are connected in parallel through

the dampers to the pulse shaper

linkage at $M_{m}$ and the load on the

pulse shaper is $\mathrm{n} \cdot \mathrm{F}_{\mathrm{o}}$.

\section{Charging the Blumlein Pulse Shaper}

At this point a new

schematic diagram (Figure B2) of

the circuit can be drawn showing

the Marx generator in its

triggered ( $f a s t$ ) configuration.

With the pulse shaper switch open,

there is no discharge path for the

charge supplied by the Marx

generator.
A new simplified linkage

shows the Marx generator after all

the cables have been cut (Figure

B2). The load is applied to the

pulse shaper linkage through the

main switch fracture link. In

this Figure both the Marx

generator and the $\mathrm{B}$ Jumle in pulse

shaper are shown as simple

equivalent spring-mass systems. 

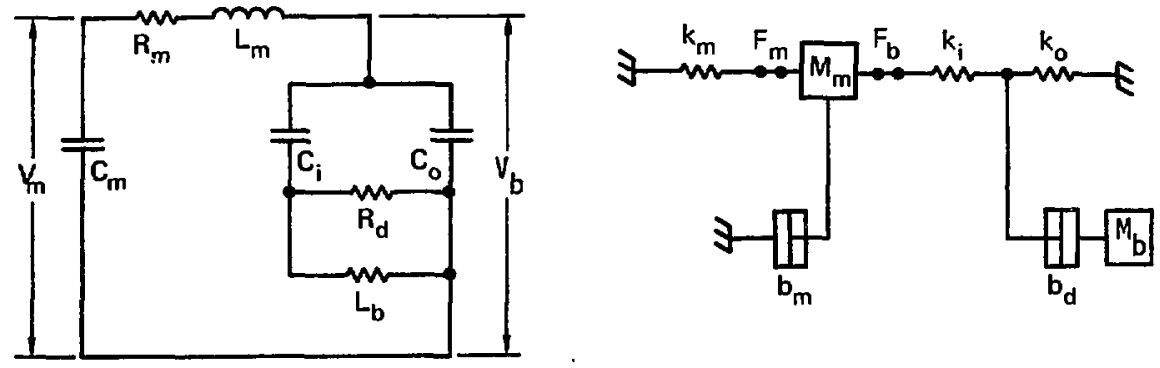

Figure B2. Equivalent systems for the time period during which the Marx generator potential is applied to the pulse shaper but before the main pulse shaper switeh has functioned. The appropriate equations and definitions follow.

$$
\begin{aligned}
& C_{\mathrm{m}}=\mathrm{n} \text { capacitors in series } \\
& =\frac{1}{1+1}=\frac{C}{n} \\
& -+-+\cdots+- \\
& C \quad \mathrm{C} \quad \mathrm{C} \\
& \mathrm{V}_{\mathrm{m}}(0)=\mathrm{n} \cdot \mathrm{V}_{\mathrm{o}} ; \mathrm{V}_{\mathrm{b}}(0)=0 \\
& R_{m}={ }^{*} \text { ine resistance in the } \\
& \text { Mürx generator } \\
& \mathrm{L}_{m}=\text { inductance in the Marx } \\
& \text { circuit } \\
& C_{1}=\text { capacitance between the } \\
& \text { inner and middle con- } \\
& \text { ductors of the pulse shaper } \\
& \mathrm{C}_{0}=\text { capacitance between the } \\
& \text { middle and outer con- } \\
& \text { ductors of the pulse shaper } \\
& R_{d}=\text { diode resistance (very } \\
& \text { high until conduction } \\
& \text { starts) } \\
& L_{b}=b a l a n c e \text { inductance to } \\
& \text { let } C_{i} \text { charge without } \\
& \text { conducting in the diode }
\end{aligned}
$$

$$
\begin{aligned}
\mathbf{k}_{\mathrm{m}} & =\mathrm{n} \text { springs in parallel } \\
& =\mathrm{n} \cdot \mathrm{k}
\end{aligned}
$$

$F_{m}(0)=n \cdot F_{o} ; F_{b}(0)=0$

$b_{m}=$ general dissipation in the Marx linkage

$M_{m}=$ lumped mass of the . Marx linkage

$k_{i}=$ stiffness of the "inner" rod of the pulse shaper

$k_{0}=$ stiffness of the "outer" rod of the pulse shaper

$\mathrm{b}_{\mathrm{d}}=$ diode damping (very high static friction - low dynamic friction)

$\mathrm{M}_{\mathrm{b}}=\mathrm{balance}$ mass to let $k_{\text {o }}$ recieve energy without moving the diode damper 
The circuit shown is

underdamped and the stored energy

will alternately charge and

discharge the pulse shaper

section. After noting that $L_{b}<L_{m}$

the circuit can be further

simplified to that shown in Figure

B3.
The motion of the linkage is

underdamped. Mass $M_{b}$ is purposely chosen to be much less than $M_{m}$ which simplifies the linkage for this phase of the operation to that shown in Figure B3. The system will oscillate with the energy being transferred alternately between $k_{b}$ and $k_{m}$.

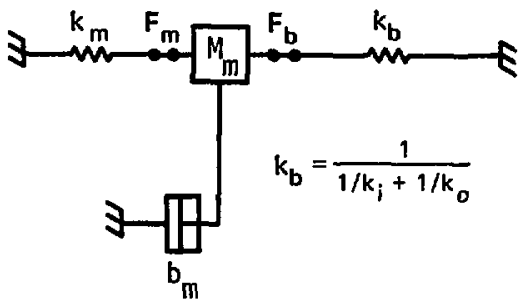

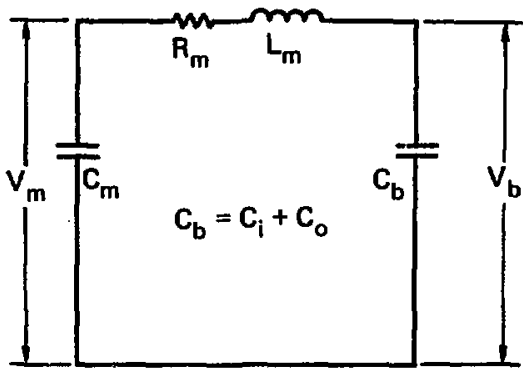

Figure.B3. Simplified schematic diagram used in determining the characteristics of energy transfer between the Marx generator and the Blumlein pulse shaper.

The systems shown in Figure B3 are for a simple damped oscillator with one degree of freedom. The equations for both the electrical and mechanical versions are easily derived and are found in most 
introductory textbooks on dynamic response. They are repeated here to show that the dynamic response is indeed identical for both systems. Solving for the voltage or force applied to the pulse shaper yields:

$$
\begin{aligned}
& V_{b}=n V_{\circ} \frac{C_{e q}}{C_{b}}\left[1+A e^{-\beta t} \sin (\omega t+\varphi)\right] \\
& c_{e q}=\frac{c_{m} c_{b}}{c_{m}+c_{b}} \\
& \beta=\frac{\mathrm{R}_{\mathrm{m}}}{2 L_{m}} \\
& A=\sqrt{\left(\begin{array}{l}
\beta \\
- \\
\omega
\end{array}\right)^{2}+1} \\
& \omega=\sqrt{\frac{1}{L_{m} C_{e q}}-\beta^{2}} \\
& \varphi=\sin ^{-1}\left(\begin{array}{c}
-1 \\
- \\
A
\end{array}\right)
\end{aligned}
$$

If $\beta$ approaches zero, which signifies very little resistance (damping) or $\omega$ approaches inf inity signifying a very high frequency system, the maximum $v_{b}$ can be approximated by:

$$
\mathrm{v}_{\mathrm{bmax}} \rightarrow \underset{\substack{\beta \rightarrow 0 \\ \omega \rightarrow \infty}}{\rightarrow} \frac{\mathrm{C}_{\mathrm{m}}}{\mathrm{C}_{\mathrm{m}}+\mathrm{C}_{\mathrm{b}}} \cdot 2 n \mathrm{v}_{0}
$$

$$
\begin{aligned}
& F_{b}=n F_{0}{ }_{k_{e q}}^{k_{b}}\left[1+A e^{-\beta t_{i n}} \sin (\omega t+\varphi)\right] \\
& k_{e q}=k_{m}+k_{b} \\
& \beta=\frac{b_{m}}{2 M_{m}} \\
& A=\sqrt{\left(\begin{array}{l}
\beta \\
\omega
\end{array}\right)^{2}+1} \\
& \omega=\sqrt{\frac{k_{e q}}{M_{m}}-\beta^{2}} \\
& \varphi=\sin ^{-1}\left(\begin{array}{l}
-1 \\
A
\end{array}\right)
\end{aligned}
$$

If $\beta$ approaches zero or $\omega$ approaches infinity as in the electrical case, the peak force developed in the Marx-Blumlein combination will be:

$$
\mathrm{F}_{\mathrm{bmax}} \rightarrow \underset{\substack{\beta \rightarrow 0 \\ \omega \rightarrow \infty}}{\rightarrow} \frac{k_{\mathrm{b}}+\mathrm{k}_{\mathrm{b}}}{\mathrm{kF}_{\mathrm{a}}}
$$


Note that for $c_{m}>c_{b}, V_{b \text { max }}$ has $a$ theoretical limit of twice the voltage developed in the Marx generator. In reality $V_{b m a x}$ reaches between 1.5 and 1.7 times $n V_{0}$.

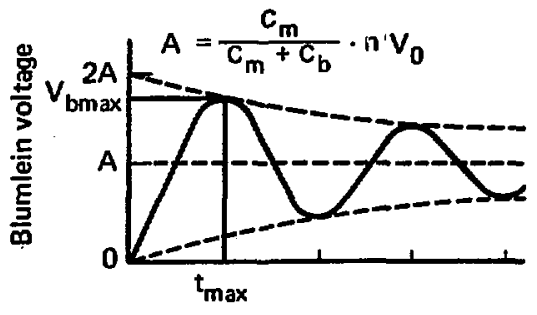

Time

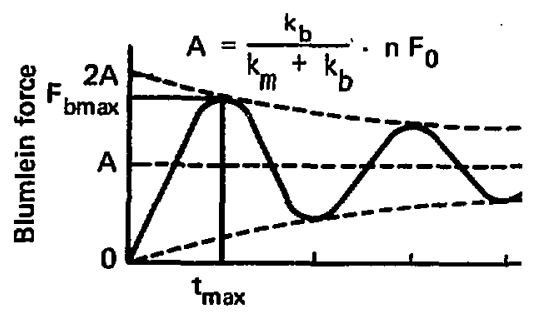

Time

Figure B4. The voltage/force developed in the capacitor/spring of a single degree of freedom system (such as the one in Figure B3) plotted as a function of time. The main pulse shaper switch is set to operate as close to the time of maximum energy storage ( $t_{\max }$ ) as is reliably possible.

The voltage applied to the

Blumlein capacitor, $C_{b}$ rises from zero to a maximum at $t_{\max }$. The circult is designed to minimize resistive losses (low $\beta$ ), maximize
The force in spring $k_{m}$ causes mass $M_{m}$ to accelerate. The acceleration continues until the force in $k_{m}$ is balanced by forces. developed in $k_{b}$ and $b_{m}$. If $k_{b}$ is 
the frequency (high $\omega$ ), and to have $C_{m}$ appropriately large in an effort to maximize energy stored in the pulse shaper in the first cycle of operation. The main switch of the pulse shaper is set to start conducting near this maximum. In practice the breakdown voltage of the main switch, which is a massive sparkgap controllable with a variable charge of sulfur hexafluride, is set for the highest reliable breakdown voltage. If the pulse shaper fails to fire and the energy oscillates back and forth into the Marx generator, serious damage to the Marx generator capacitors can result. large compared to $\mathrm{k}_{\mathrm{m}}$, the force in $k_{m}$ is nearly constant. If the damping, $b_{m}$. is small the acceleration will be zero when the force in $k_{b}$ is the same is that in $k_{m}$. The velocity at this point in time is not zero and $M_{m}$ continues to add energy to $k_{b}$ unt $j$ l it comes to rost. The symmetry of the problem indicates, as do the equations, that $M_{m}$ will be at rest when the total force in $k_{b}$ is twice that in $k_{m}$. 


\section{Discharge of the Blumlein Pulse Shaper}

\section{Closing the Blumlein switch}

changes the conf $i$ guration of the pulse shaper so that a voltage pulse is developed across the diode resistor, $R_{d}$ (Figure $B 2$ ). The discharge of the pulse shaper through $R_{d}$ is governed by the physical characteristics of the triaxial conductor. An equivelent circuit for the Blumlein operation is shown in Figure 85.

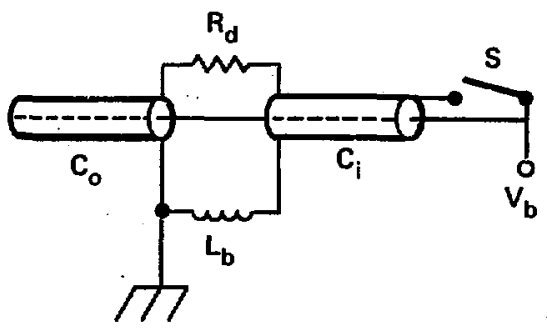

Figure B5. Blumlein triaxial pulse shaper equivalent circuit

When the switch, $S$, is closed, a short.circuit is applied to the end of the inner conductor. The end of the conductor is unable
At the predesignated force

the fracture link (Figure B1) connecting the Marx linkage to the pulse shaper linkage will

separate. At this instant, the end of the "inner" conductor (rod) can no longer support a stress and a compressive stress wave begins to travel along the rod toward the diode link. The cross sectional area of the Blumlein rods is $A_{b}$ and $\sigma_{b}$ is the ratio, $F_{b} / A_{b}$. The stress behind the wave must be zero to achieve the condition that the stress at the end is zero, so the magnitude of the wave is $-\sigma_{b \max }$ which is equal to $-F_{b \max } / A_{b}$ (Figure 84 ). The release of energy in the stress waves is fast enough that the balance mass, $M_{b}$, will remain nearly stationary and can be considered fixed. The left moving compressive wave lakes $t_{0}$ 
to maintain a voltage potential and a wave results with a

magnitude that will cancel the initial voltage, $v_{\text {bmax }}$ (Figure B4). This wave travels from the switch to the diode in $t_{0}$ seconds where part of the wave is transmitted through $R_{d}$ to the outer conductor and part is reflected back into the inner conductor. When the wave first arrives at $R_{d}$, it instantaneously develops a voltage $-V_{\text {bmax }}$ across the diode. This process takes place so quickly that the balance inductor, $\mathrm{L}_{b}$. appears to be an open circuit. At this point $R_{d}$ begins to conduct, reflecting part of the wave's energy back into the inner conductor and transmitting the rest through $R_{d}$ to the outer conductor pair.

Equations for the exact magnitudes of the transmitted and

seconds to arrive at the diode damper link. At this link the forces must add to zero and the ends of the bars and the damper must have equal velocities. The velocity change in the rod, $\Delta v$, is equal to the magnitude of the wave, $\Delta \sigma$, divided by the impedance, $z^{\dagger}$.

$$
\begin{aligned}
& \Delta \sigma_{\mathrm{i}}=\mathrm{z}_{\mathrm{i}} \cdot \Delta \mathrm{v}_{\mathrm{i}} \\
& \Delta \sigma_{\mathrm{o}}=\mathrm{z}_{\mathrm{o}} \cdot \Delta \mathrm{v}_{\mathrm{o}} \\
& " \sigma_{\mathrm{d}} "=\mathrm{F}_{\mathrm{d}} / \mathrm{A}_{\mathrm{b}}=\mathrm{z}_{\mathrm{d}} \cdot \mathrm{v}_{\mathrm{d}} \\
& \left(\mathrm{z}_{\mathrm{d}}=\mathrm{z}_{\mathrm{d}}^{\prime} / \mathrm{A}_{\mathrm{b}}\right)
\end{aligned}
$$

So that stress can be equated at the diode, the: diode impedance, $z_{d}$, is divided by the area of the pulse shaper rods, $A_{b}$, to get an equivalent impedance, $z_{d}$. The stress compatability criteria and the velocity continuity criteria at the damper link result in:

$$
\begin{aligned}
\sigma_{i_{I}} & =\text { incident wave in inner rod } \\
\sigma_{i_{R}} & =\text { wave ref lected back into } \\
& \text { inner rod } \\
& =\left(\frac{z_{d}+z_{0}-i_{i}}{z_{i}+z_{o}+z_{d}}\right) \sigma_{i}
\end{aligned}
$$

$\uparrow$ - The symbol $z$ is used to denote impedance relating effort per unit area to flow. The symbol $z^{\prime}$ relates effort to flow directly. 
reflected waves can be derived

using the characteristic

impedances of the inner and outer

conductors along with the

resistance of the diode. Figure

B6 shows the voltage distribution

in the Blumlein network at three

times during the pulse. The

current through the diode must

equal the current flow in each

conductor because there are no

current sources in the diode. The

voltage drop across the diode must

be the difference in the

potentials at the ends of the

inner and outer conductors. This

is precisely analogous to saying

that the forces on the damper link

in the mechanical analog must sum

to zero and that the velocities

are equal.

The detaits of the electrical reflections and transmissions can be inferred from the following

$$
\begin{aligned}
\sigma_{\boldsymbol{O}_{T}} & =\text { wave transmitted into } \\
\text { outer rod } & \left(\frac{2 z_{0}}{z_{i}+z_{0}+z_{d}}\right) \sigma_{i}
\end{aligned}
$$

The force on the damper $b_{d}$ is the difference between the forces exerted by the "inner" and "outer" conductors. From Figure B6 it can be seen that after the first

transmission the difference in stresses is:

$$
\sigma_{i}-\sigma_{0}=-\sigma_{b \max }\left(\frac{2 z_{d}}{z_{i}+z_{o}+z_{d}}\right)
$$

The force on the damper is this stress $t$ imes $\hat{A}_{b}$. The velocity of the damper link can be calculated as follows:

$$
\begin{aligned}
v_{d} & =\frac{A_{b}\left(\sigma_{i}-\sigma_{0}\right)}{z_{d}} \\
& =\frac{-2 \cdot \sigma_{b \max }}{z_{i}+z_{0}+z_{d}}
\end{aligned}
$$

The waves shown in Figure B6(b) will reflect from the ends of the bars. The wave in the "inner" rod 


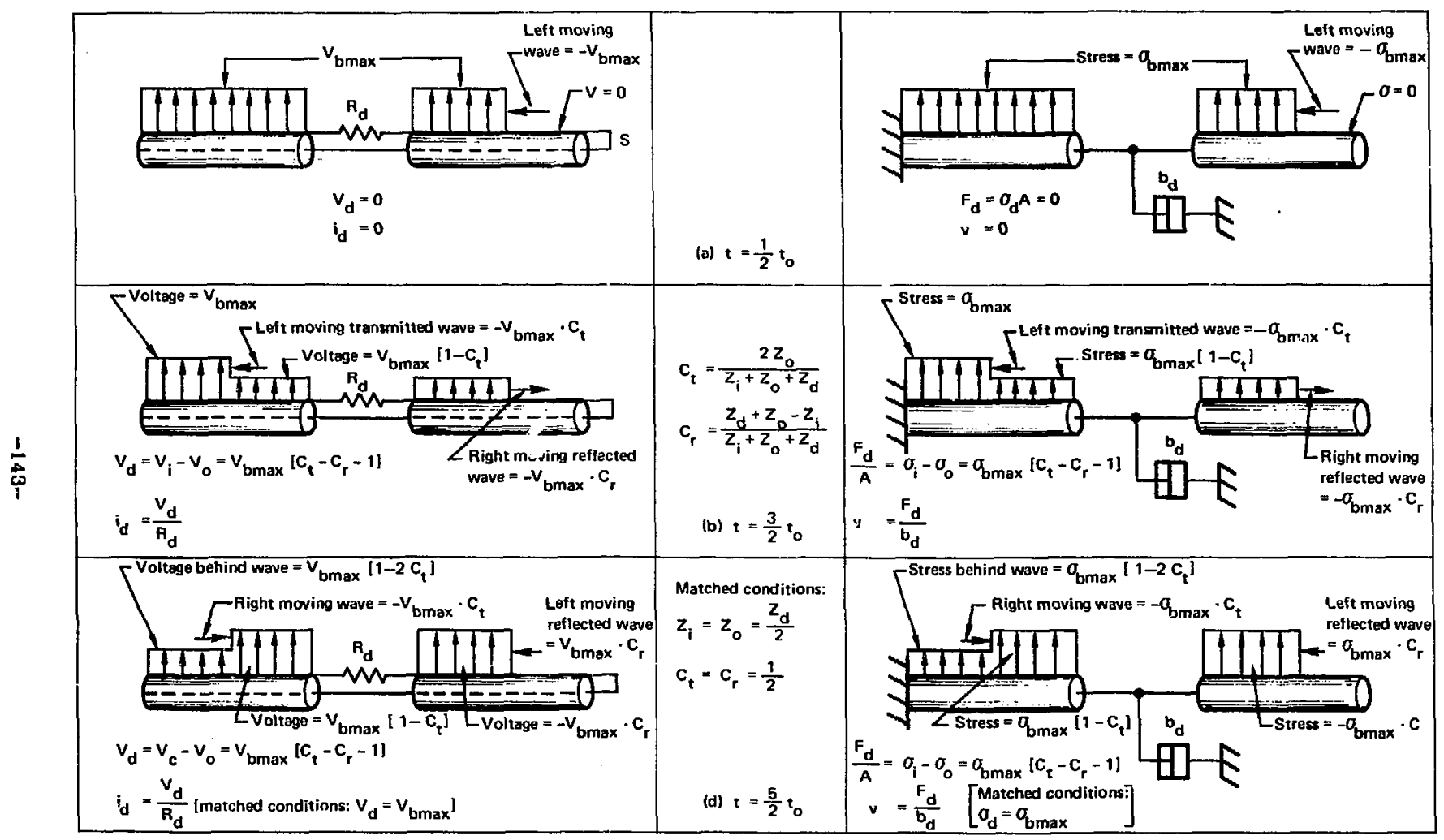

Figure B6. The dynamic response of the analogous systems depicted at three instants in time. The transmission and reflection coefficients and the /response of diode damper are derived. 
detailed treatment of the mechanical system. will reflect from a free end, and the wave in the "outer" rod will reflect from a fixed end. These reflected waves will return to the damper $l$ ink and create a new net force on the damper. In general, the force will remain constant for time increments of $2 \cdot t_{0}$ and change in a series of steps until all of the energy has been dissipated in the damper.

\section{E. Impedance Matching for Optimuin Response.}

The object of pulse shaping is to maximize the energy dissipation in the shortest possible pulse. If the two stress waves returning from their reflections at the ends of the pulse shaper rods (Figure B6(c)) are equal in magnitude and opposite in sign, then they will cancel each other. If in addition the stress behind both waves is zero, then no new waves will be generated at the damper or at the ends of the rods. This results in a single pulse $2 t_{0}$ seconds in duration. Making both rods equal in length provides simultaneous arrival of the waves, and setting:

$$
z_{i}=z_{0}=z_{d} / 2
$$

which is derived from:

$$
\begin{array}{ll}
\sigma_{b \max }\left(1-c_{\imath}\right)=0 & \text { [stress behind left wave is zero] } \\
\sigma_{b \max } \cdot c_{r}=-\left(-\sigma_{b \max }+c_{t}\right) & \text { [arriving stress waves cancel] }
\end{array}
$$


insures that the stresses meet the above criteria. These are the familiar "matched impedances" and the force history on the damper link is plotted in Figure B7.

The power dissipation during the first pulse is:

$$
\begin{aligned}
P_{d}=F_{d} \cdot v_{d} & =\left[\frac{-\sigma_{b \max } \cdot A_{b} \cdot 2 z_{d}}{z_{b}+z_{o}+z_{d}}\right] \cdot\left[\frac{-\sigma_{b m a x} \cdot 2}{z_{i}+z_{o}+z_{d}}\right] \\
& =4 \sigma_{b \max }^{2} \cdot\left[\frac{z_{d}}{z_{i}+z_{o}+z_{d}}\right] .
\end{aligned}
$$

By differentiating $P_{d}$ with respect to $z_{d}$ and seitiag the result equal to zero, the maximum power is found to occur when $z_{d}$ equals $\left(z_{i}+z_{o}\right)$. This is confirmation of the intuitive reasoning that if the energy is dissipated in one pulse, i.e. at matched impedances, then the power must be at a maximum. One can also see that if the conductor impedances $\left(z_{i}+z_{o}\right)$ can be made small, then the power is increased.

\section{F. Comments on the Coaxial Pulse Shaper Circuit}

Figure $B 8$ shows the system and the response of a mechanical equivalent for the co-axial pulse shaper circuit. For this system a "matched" condition exists when $z_{p}$ (the pulse shaper impedance) is equal to $z_{d}$. An inspection of the equations shows that at "match" the pulse will again be $2 t_{0}$ seconds wide, but that the magnitude will be $\frac{1}{2} \sigma_{b m a x}$. For the same maximum Marx generator output, the mean electron energy level at maximum power dissipation will be only half that available with the Blumlein configuration. 


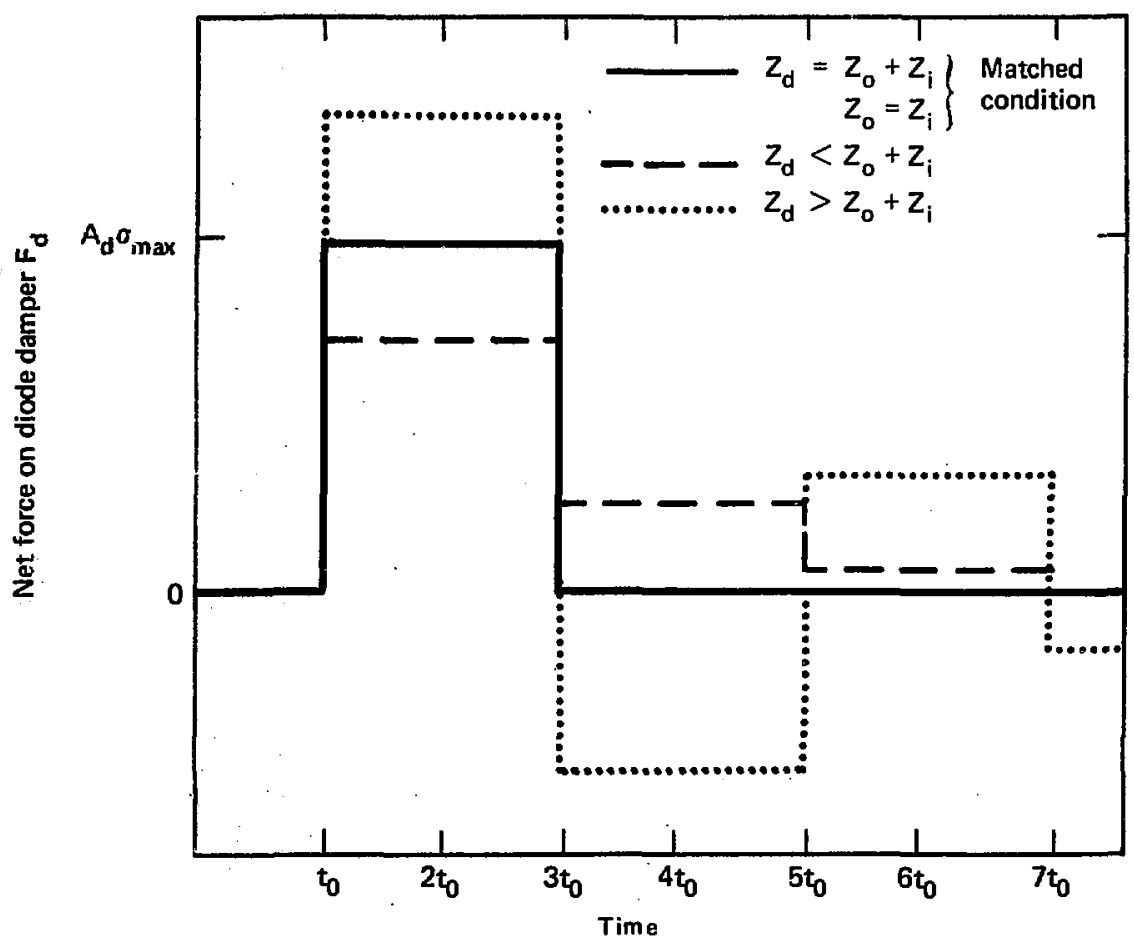

Figure Br. The net force on the damper plotted versus time for "matched", "overmatched" $\left(z_{d}>z_{1}+z_{0}\right)$, and "undermatched" $\left(z_{d}<z_{1}+z_{0}\right)$ damper impedances. 


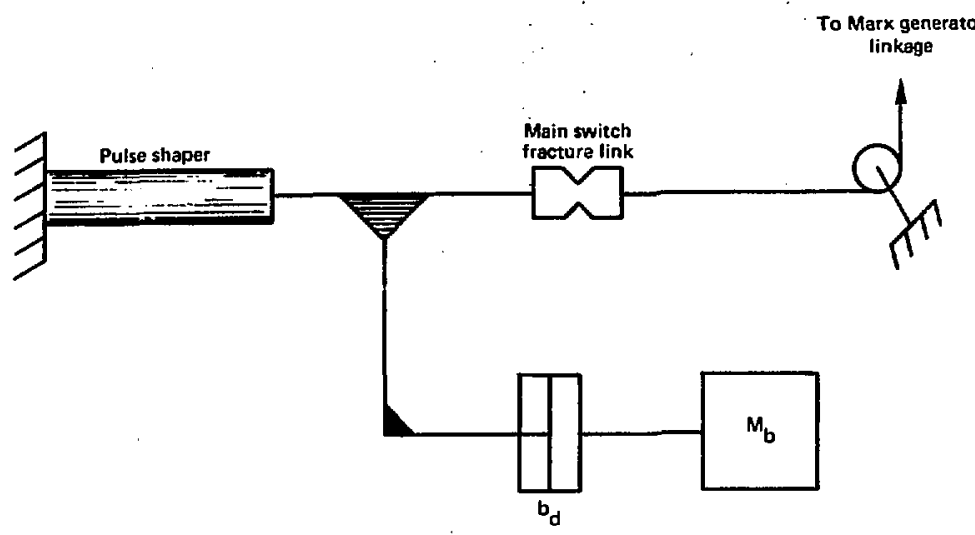

Figure B8a. The mechanically equivalent linkage for a co-axial pulse shaper system.

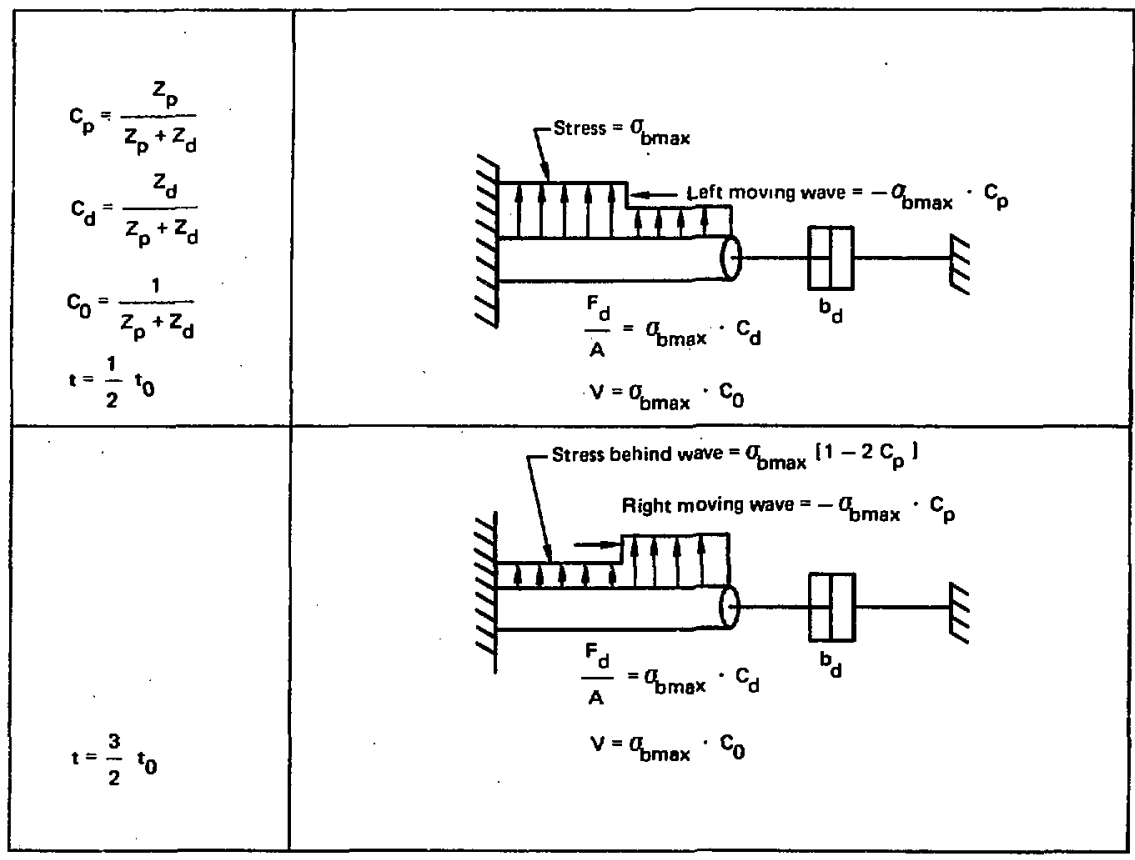

Figure B8b. The response of the co-axial pulse shaper at two instants in $t$ ime. 


\section{APPENDIX C.}

Summary of Computer Programs Written to Aid in the

Interpretation and Presentation of the Data

\section{SEGCAL}

Purpose: SEGCAL was written to provide a fast means for scaling and displaying the results of an e-beam experiment in which a segmented calorimeter was used to measure the beam energy. A teletype terminal was used to convert the thermocouple data in millivolts to energy fluence in calories per square centimeter. The data was then displayed on the terminal in a facsimile of the calorimeter segment positions. Data corresponding to the maximum, minimum, average, and standard deviation of the fluence pattern was also typed out.

Function: The operator would establish the initial and maximum thermocouple output in millivolts for each of the calorimeter segments. This data as well as the reference junction temperature and calorimeter designation number were entered into a computer with a teletype terminal in a free field format. SEGCAL searched a library file for the calorimeter specified to get specific heat data for the segments, thermocouple type, and spatial format of the segments. The thermocouple data was converted to temperature using polynomial fits to thermocouple reference charts. The change in temperature was converted to an energy deposition using the specific heat data, and finally to fluence by dividing by the area of each segment. 
Using data from the library file, the fluence data was printed on the teletype terminal in an array corresponding to the calorimeter segment layout (see Figure 8 ). If requested in the input, a table of the data from each segment would be printed. Contours of constant fluence could be madc on a computer generated graph and sent to a hard copy device for processing.

\section{DIGIPLT}

Purpose: DIGIPLT reads, scales, and plots records of digital data. When used to process carbon gage data, it established the pulsed power supply parameters from the data corresponding to the initial conditions of the gage circuit. Plots of the stress at the carbon gage versus time for several data records could then be superimposed nn a single plot for eomparison purposes.

Function: After reading an instruction deck, the appropriate file and record of input data were read. The equations for the pulsed power supply in Section 11 were used to solve for the actual values of $R_{1}$ and $R_{2}$. The same equations were then used to get $\Delta R / R$ versus $t$ ime from $V(t)$. The resistance change data was scaled using the third order polynomial fit to the gage sensitivity (Figure 19). Other typus of scaling were available and adding new scaling subroutines was routine.

Input :

A. Instruction deck:

1) Header card with output disposition information

2) Title and function switches 
3) Plot label (x-axis)

4) Plot label (y-axis)

5) Mapping parameters for output plots

6) Pointers to file and record for digital data plus scaling instructions. and scaling data.

Card 6) was repeated to get additional data on the same plot. Returning to card 2) started a new plot.

B. Digita! data: Digitized data from oscilloscope trace photographs. The first card was the name of the record and following cards contained voltage versus time data pairs. The record was terminated by a time less than $-10^{10}$ seconds. Additional records followed the first or were resident in separate files.

Output: The output were graphs of the processed data with stress in kilobars plotted versus time. Multiple traces on a single frame were possible. When desired, the traces were integrated between the limits of the time axis with the result printed on the plot frame.

\section{CGMODEL}

Purpose: CGMODEL was used to evaluate the effect of the filter connected between the carbon gage and its power supply. Predicted stresses in the carbon gage were converted to an ouput signal from the power supply bridge circuit. These signals were compared with similar data. when the filter was removed from the model. A comparison showed that the filter had a minimal influence on the data signal. The magnitude was altered by less than $10 \%$ and the pulse was delayed by less than $75 \mathrm{~ns}$. 
Function: Stress data from KOVSPALL were converled to resistance change $\left(\Delta R_{B} / R_{B}\right)$ versus time using the data and equation in Figure 19 . The state equations (eq. 11) in Section IV were intefrated using a fourth order Runge-Kutta method with a time varying coefficient in the $\underline{\underline{A}}$ matrix. When $\mathbf{R}_{\mathrm{g}}$ varied, disturbances were propagated through the filter circuit and the effect at the power supply termination resistance, $R_{t}$, was calculated and plotted.

Input :

A. An instruction deck consisting of a series of cards with the name of the input data file and the ordinate plotting limits for each output graph.

B. A series of files of data specified by the names on the instruction cards. These files were created by KOVSPALL and a header card was added by the user. The header card specified the title, the filter capacitance and inductance, the power supply voltage, and the integration parameters. The following cards (crealed by KOVSPALL) contained the stresses for up to 10 zones at a series of times specified in the input to KOVSPALL.

Output: For each instruction card a graph of the carbon gage power supply output signal (volts) was plotted versus time.

\section{VELOC ITY}

Purpose: VELOCITY provided a means by which the $35 \mathrm{~mm}$ roll film data could be converted reliably to velocity dato for further analysis. 
The curvature of the tamper required an averaging process to establish the mean velocity over the tamper surface.

Function: After reading the instruction card, all of the tamper coordinate data were read and stored. This data described the tamper profile in the $x, y$ plane where $x$ was the direction of tamper motion and $y$ was the vertical direction across the tamper surface. The z-direction was horizontal across the tamper surface. The $x$ position at each of 50 equally spaced y coordinates was obtained by linear interpolation of the input data. The curvature in the z-direction was assumed to be the same as that in the $y$-direction and a average $x$ position $(\bar{x})$ was calculated. After all the frames had been processed in this manner, two velocities were calculated. The average velocity was computed by taking the difference between $\bar{x}$ values for successive frames and dividing by the time interval between frames. The maximum velocity of the tamper was calculated using the maxmimum change in $x$ on successive frames and dividing by the time per frame.

Input :

A. An instruction deck consisting of cards each with the name of a datafile, the time between frames, and the time for the first frame.

B. Digital Data from the enlargements of the framing camera films. Each record contained up to 250 pairs of $x, y$ coordinate data describing the tamper profile position. The end of a record was flagged with a negative $x$ value. Additional records were 
processed (one for each frame) until a zero was encountered for the first $x$ position in a record.

Output:

A. A series of small replicas of the original $35 \mathrm{~mm}$ frames were plotted. These replicas were compared with the original frame to check for errors in converting the data to digital form.

B. The maximum and average velocities of the tamper surface were plotted on the same frame as a function of time for the time span covered by the number of frames initially processed. 


\section{APPENDIX D. \\ Summary of Set-up Parameters and Diagnostic Measurements \\ For All Electron Beam Tests in this Experiment}

This experimental program consisted of 384 electron beam firings. Configuring the electron-beam machine and developing the experimental apparatus for the test required 262 runs. The first 107 (shot no. 4666 - no. 5319) were used to determine the e-beam machine operating parameters that would maximize a reasonably uniform energy distribution and remain stable at those conditions. Toward the end of that phase some instrumentation system checkout was begun. The following 155 runs (no. 6027 - no. 7099) were used to find and correct problems with the experiment diagnostic equipment. During this second phase the output of the e-beam was monitored and changes were made to the operating parameters in order to mainlain the energy at the desired levels. The final 122. shots (no. 7800 - no. 7921 ) were the series that provided the 21 valid data runs.

The standard diagnostic measuremenls obtained from each run were collected and tabulated with comments. This information is available from the author. 